LBL-38145

\title{
A pQCD-based Approach to Parton Production and Equilibration in High-Energy Nuclear Collisions
}

\author{
XIN-NIAN WANG \\ Nuclear Science Division, Mailstop 70A-3307 \\ Lawrence Berkeley National Laboratory \\ University of California, Berkeley, CA 94720 USA
}

\section{Contents}

\begin{tabular}{lll}
\hline 1 & Introduction & 3
\end{tabular}

\begin{tabular}{|lll}
2 & Parton Scatterings in Hadron-Hadron Collisions & 7
\end{tabular}

2.1 Pomeron Exchange and Minijet Production . . . . . . . . . . . . . . . 7

2.2 Cross Sections: Soft vs. Hard . . . . . . . . . . . . . . . . . . . . . 11

2.3 Modeling the Soft Interactions . . . . . . . . . . . . . . . . . . 15

2.4 Minijets and Transverse Flow . . . . . . . . . . . . . . . . . . . . . . 17

2.5 Resolving Minijets in Hadronic Interactions . . . . . . . . . . . . . . 21

3 Parton Production in Nucleus-Nucleus Collisions 24

3.1 Nuclear Shadowing of Parton Distribution Functions . . . . . . . . . 25

3.2 Disappearance of Cronin Effect at High Energies . . . . . . . . . . . . 34

3.3 Monte Carlo Simulations . . . . . . . . . . . . . . . . . . . . 38

3.4 Space-Time Structure of Parton Production . . . . . . . . . . . . . . 45

3.4 .1 Space-time history . . . . . . . . . . . . . . . . 45

3.4 .2 Local isotropy and chemical composition . . . . . . . . . . . 51

3.4 .3 Multiple initial parton scatterings ? . . . . . . . . . . . 55

*This work was supported by the Director, Office of Energy Research, Division of Nuclear Physics of the Office of High Energy and Nuclear Physics of the U.S. Department of Energy under Contract No. DE-AC03-76SF00098 and U.S. - Hungary Science and Technology Joint Fund J. F. No. 378 
$\begin{array}{lll}4 & \text { Parton Equilibration } & 56\end{array}$

4.1 Initial Conditions: a Hot and Undersaturated Gluonic Gas . . . . . . 57

4.2 Master Rate Equations . . . . . . . . . . . . . . . . . . . . . . 58

4.3 Parton Equilibration Rates . . . . . . . . . . . . . . . . . . . . . . . . 60

4.4 Evolution of the Parton Plasma . . . . . . . . . . . . . . . . . . . 63

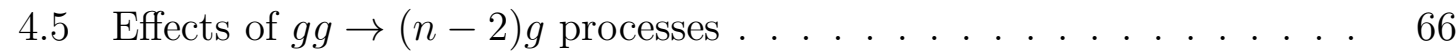

5 Hard Probes of the Equilibrating Parton Plasma 68

5.1 Open charm production . . . . . . . . . . . . . . . 69

5.1 .1 Thermal production during equilibration . . . . . . . . . . . . 69

5.1 .2 Pre-thermal production . . . . . . . . . . . . . 71

5.1 .3 Initial fusion . . . . . . . . . . . . . . . . . . . . . . . . . . . . . . . . 72

5.2 Dilepton and Photon Production . . . . . . . . . . . . . . . 76

$5.3 \quad J / \psi$ Suppression . . . . . . . . . . . . . . . . . . . 80

5.4 Jet Quenching and Monojet Production . . . . . . . . . . . . . . . 85

5.4 .1 Energy loss of a fast parton in QGP. . . . . . . . . . . . 86

5.4 .2 Suppression of dijets . . . . . . . . . . . . . . . . . 92

5.4 .3 Monojet production . . . . . . . . . . . . . . . . . . 95

\begin{tabular}{lll}
\hline 6 & Summary and Discussion & 97
\end{tabular}

\begin{abstract}
A pQCD-based model for parton production and equilibration in ultrarelativistic heavy-ion collisions is reviewed. The model combines pQCD processes including initial and final state radiations together with string phenomenology for nonperturbative soft processes. Nuclear effects on the initial parton production, such as multiple parton scattering and nuclear shadowing of parton distribution functions are considered. Comparisons with existing data are made and further tests of the model to constrain model parameters are proposed. With the obtained space-time history of the parton production, evolution of the minijet gas toward a fully equilibrated parton plasma is studied. Direct probes of the early parton dynamics, such as pre-equilibrium photon and dilepton production, open charm production, $J / \psi$ suppression and jet quenching are also reviewed.
\end{abstract}




\section{Introduction}

According to the Big Bang theory, during the early stage of the evolution of our universe, there existed a state of matter consisting mainly of unbound quarks and gluons at a temperature of about $200 \mathrm{MeV}$. As the universe expanded and cooled down, it went through a phase transition and eventually evolved to what we see today. Such a phase transition was predicted [1, 2] shortly after Quantum Chromodynamics (QCD) was established as the fundamental theory for strong interactions. In this theory, quarks, which have three types of color charges, interact via non-Abelian gauge bosons, i.e., gluons. Because of the non-Abelian nature of strong interactions, quarks and gluons are confined to hadrons under normal circumstances. However, hadronic matter under extremely dense and hot conditions will go through a phase transition to form a quark-gluon plasma in which quarks and gluons are no longer confined to the size of a hadron. Lattice gauge studies of QCD at finite temperatures have indeed found such a phase transition, though the nature of the transition has not yet been clarified [3].

To recreate the early universe and experimentally verify the QCD phase transition in the laboratory, it was proposed that nuclei be accelerated to extremely high energies and then allowed to collide with each other. During the collisions, both high baryon densities and high temperatures might be reached. Thus, a quark-gluon plasma could be formed which will subsequently go through the phase transition and hadronize into particles in the final states. During the last decade, heavy-ion experiments have been done at the AGS of BNL and the SPS of CERN with $E_{\text {lab }} \approx 14$ and $200 \mathrm{AGeV}$, respectively. The results from those experiments [i] have demonstrated extremely rich physics which cannot be explained by simple extrapolation of $p p$ collisions. However, up to now, there is no unambiguous evidence for the existence of a quark-gluon plasma over a significantly large space-time region. While experiments at AGS and SPS and their analyses continue, new experiments [5] have been planned at RHIC of BNL and LHC of CERN with $E_{\mathrm{cm}}=200 \mathrm{AGeV}$ and $5.5 \mathrm{ATeV}$, respectively. What I would like to emphasize in this review is that heavy-ion collisions at these ultrarelativistic energies will demonstrate a completely new dynamics which is not accessible at the present energies.

The key issue here is the nuclear structure at different scales. When the transverse momentum transfer involved in each nucleon-nucleon collision is small

$\left(p_{T} \lesssim \Lambda_{\mathrm{QCD}}\right)$, effective models based on, e.g., meson-exchange and resonance formation are sufficient to describe multiple interactions between hadrons in which the parton structure of the hadrons cannot yet be resolved. These coherent (with respect to the partons inside the hadron) interactions lead to collective behavior in low-energy 
heavy-ion collisions as were first observed in the Bevalac experiments [6] and recently at the AGS energies [7]. However, when $p_{T}$ becomes large enough to resolve individual partons inside a nucleon, the dynamics is best described on the parton level via perturbative QCD (pQCD). Though hard parton interactions occur at CERN-SPS energies $(\sqrt{s} \lesssim 20 \mathrm{AGeV})$, they play a negligible role in the global features of heavyion collisions. However, at collider energies $(\sqrt{s} \gtrsim 100 \mathrm{AGeV})$ the importance of hard or semihard parton scatterings is clearly seen in high-energy $p p$ and $p \bar{p}$ collisions [ [8]. They are therefore also expected to be dominant in heavy-ion collisions at RHIC and LHC energies [9, 10]. These hard or semihard interactions happen on a very short time scale and they generally break color coherence inside the individual nucleons. After the fast partons pass through each other and leave the central region, a dense partonic system will be left behind which is not immediately in thermal and chemical equilibrium. The partons inside such a system will then further interact with each other and equilibration will eventually be established if the interactions are frequent enough among a sufficiently large number of initially produced partons. Due to the asymptotic behavior of QCD, production rates of hard and semihard partons are calculable via perturbative QCD during the initial stage of heavy-ion collisions. The color screening mechanism in the initially produced dense partonic system makes it also possible to use pQCD to investigate thermal and chemical equilibration of the system.

Currently, there are many models to describe the coherent hadronic interaction in which parton degrees of freedom are not important yet. In these models, hadrons remain as individual entities and particle production is mainly through resonance formation. Indeed, most of the experimental data at AGS energies can be explained by RQMD [11] and ARC [12 Monte Carlo simulations. As the colliding energy increases, partons inside nucleons become more relevant as the basic constituents of the interaction. However, nonperturbative soft interactions still dominate the collision dynamics in the energy range $\sqrt{s} \lesssim 100 \mathrm{AGeV}$. In order to describe particle production in this energy range, phenomenological string models [13, 14, 15, [16] have been developed and they can explain well the global properties of particle production at CERN SPS energies. The physics behind these string models is that multiple partons produced in soft interactions are not independent of each other. Coherence among them can be modeled by strings or flux tubes between leading quarks and diquarks (or antiquarks). Particle production comes from the fragmentation of the string through $q \bar{q}$ creation inside the flux tube. At collider energies, $\sqrt{s}>100 \mathrm{AGeV}$, hard and semihard scatterings, which can be calculated via pQCD, become increasingly important. Since nonperturbative physics is always present in any processes involving strong interactions, pQCD has to be combined with models of nonperturbative inter- 
actions, e.g., string models. Such pQCD-inspired models, like PYTHIA [17], ISAJET [18], describe well the high $p_{T}$ phenomena in high-energy hadronic collisions. Along the same line, M. Gyulassy and I extended this approach to high-energy heavy-ion collisions and developed the HIJING (Heavy Ion Jet INtercation Generator) Monte Carlo model [19] to take into account the physics of hard and semihard parton production. Nearly at the same time, similar models, PCM [20] and DTUNUC [21], also emerged. In particular, PCM has explicitly modeled the space-time evolution of the parton production processes and rescatterings along the lines of Boal [22]. Though hard and semihard processes become dominant at high energies, they are always accompanied by soft interactions. These soft interactions in general produce many soft final partons and take longer time $(\sim 1 \mathrm{fm} / c)$ to complete. Therefore, they cannot be modeled by simple elastic parton-parton scatterings. However, these nonperturbative interactions might be modified inside a dense partonic medium, so that a perturbative method might be sufficient to describe the evolution of the initially produced dense partonic system towards an equilibrated quark-gluon plasma.

The crux of parton dynamics in heavy-ion collisions rests in the complexity of multiple parton scatterings and the associated bremsstrahlung. An exact quantum field treatment of these multiple parton interactions must include all possible matrix elements and the interference between them. Although quantum transport theory [23, 24, 25] can in principle include all these interference effects and developments in this subject are impressive in the last few years, its applicability to heavy-ion collisions is still far from being realistic. An alternative and more realistic solution to this problem is to find ways to incorporate correctly the subtle interference phenomena in a classical parton cascade model. There are two important interference phenomena in multiple parton interactions. (1) The interference among different amplitudes of multiple parton scatterings, especially in the initial parton scatterings when the two beams of fast partons pass through each other, leads to the Glauber formula of multiple interactions [26, 27]. Thus, a semiclassical cascade cannot be used to treat the initial parton production during the overlapping period of the two colliding nuclei. The resultant interference is responsible, as I will demonstrate later, for the disappearance of the nuclear enhancement of jet production in $p A$ collisions at large $p_{T}$ and high energies. Therefore, we can neglect processes in which a parton suffers multiple hard scatterings in the initial parton scatterings. Brodsky and Lu have shown [28] that a Glauber analysis of multiple antiquark-nucleon scatterings also leads to nonperturbative shadowing of the quark distributions inside heavy nuclei. (2) The spectrum of induced gluon bremsstrahlung in multiple scatterings is also modified by the destructive interference among different radiation amplitudes, the so-called Landau-Pomeranchuk-Migdal (LPM) effect [29, 30]. In a detailed analysis [27, 31], 
one finds that the interference actually happens between two amplitudes in which the beam parton has completely different virtualities, i.e., time-like in the final state radiation of one scattering and space-like in the initial state radiation of the previous one. Therefore, a propagating parton can no longer be considered as always time-like with a decreasing virtuality in a parton cascade simulation, e.g., PCM 20].

Another aspect of parton production and interaction in ultrarelativistic heavyion collisions is that the same processes responsible for parton equilibration can also provide direct probes of the early parton dynamics and the properties of the parton gas during its evolution toward a fully equilibrated quark-gluon plasma. For example, parton scatterings in an equilibrating system also lead to dilepton, photon and charmed quark production. Since the production rates of these three processes are proportional to the product of quark-quark, quark-gluon and gluon-gluon densities, respectively, measurements of the thermal enhancement of dileptons, photons and charmed quarks can provide us with information of the quark and gluon densities in the plasma, and thus on the thermal and chemical equilibration. Interactions of an initially produced high- $p_{T}$ jet with partons inside the equilibrating plasma, in the meantime, will cause the jet losing energy. Studying of jet quenching due to energy loss can thus tell us whether and how the the parton gas is thermalized. Because of the same mechanism, $J / \psi$ is also suppressed due to its interaction with the parton gas. Since the $J / \psi$ dissociation cross section inside a deconfined partons is very different from that inside a hadronic gas, the study of $J / \psi$ suppression can then provide us with evidence for color deconfinement in the parton plasma and possibly with information on the QCD phase transition.

This review will be structured as follows: In the next section, I will review a pQCD-inspired model for multiple parton production in $p p, p A$ and $A A$ collisions. Special emphasis will be devoted to the role of hard and semihard parton collisions and their connection to Pomeron structure and soft interactions. I will also discuss the interpolation between hard and soft physics and the possible treatment of nonperturbative interactions. In Section 3, nuclear effects such as multiple initial and final state interactions and nuclear shadowing of parton distribution functions will be discussed. The Monte Carlo implementation of parton production will also be reviewed. In Section 4, I will discuss the evolution of a partonic system via pQCD, including the Landau-Pomeranchuk-Migdal (LPM) effect on induced gluon radiation. In Section 5, I will discuss the possibility of using hard processes as probes of the early parton dynamics, the formation of quark-gluon plasma formation and the QCD phase transition. In particular, photon and dilepton production, charm production, pre-equilibrium $J / \psi$ suppression, jet quenching, and monojet production will be discussed. Finally I will give a summary and discussion in Section 6 . 


\section{Parton Scatterings in Hadron-Hadron Collisions}

High-energy nuclear collisions are expected to be dominated by semihard parton collisions with transverse momentum transfers $p_{T} \gtrsim p_{0} \sim 1-2 \mathrm{GeV} / c$. The produced partons carrying such transverse momenta are often referred to as minijets. Multiple parton production has been estimated [10] to produce up to $50 \%(80 \%)$ of the transverse energy per unit rapidity in the collisions of heavy nuclei at RHIC (LHC) energies. While not resolvable as distinct jets, minijets are expected to lead to a wide variety of correlations among observables, such as transverse momentum, strangeness and fluctuation enhancements, that compete with expected signatures of a QGP. Therefore, it is especially important to calculate these background processes as reliably as possible. In addition, it has been shown that multiple mini-jet production is important in $p \bar{p}$ interactions to account for the increase of the total cross section [33] with energy, the increase of the average transverse momentum with charged multiplicity [34], and the violation of Koba-Nielsen-Olesen (KNO) scaling of the charged multiplicity distributions [17, 35].

It has long been recognized [33] that the inclusive jet cross section $\sigma_{\text {jet }}$ in $p p$ or $p \bar{p}$ collisions increases very rapidly with energy and eventually will be larger than the total inelastic cross section $\sigma_{\text {in }}$. This is because the inclusive jet cross section also contains the average number of jets per inelastic event. Thus,

$$
\sigma_{\text {jet }}=\left\langle n_{\text {jet }}\right\rangle \sigma_{\text {in }}
$$

where $\left\langle n_{\text {jet }}\right\rangle$ is the average number of hard or semihard scatterings per inelastic event. One way to include hard or semihard processes in the unitarized cross sections of $p p$ and $p \bar{p}$ is to introduce the idea of Pomeron exchanges in an eikonal formalism. I will argue that hard or semihard scatterings can be considered as the hard loops inside a Pomeron exchange, and the interpolation between hard and soft components will become clear. This will also shed some light on the modeling of the soft component.

\subsection{Pomeron Exchange and Minijet Production}

The Pomeron was introduced to describe the effective interaction between quarks and gluons [36, 37, 38]. It has been used to model hadronic cross sections, diffractive interactions and multiple particle production. In general, it is considered to be a color singlet object with the quantum numbers of a photon. It can couple both to quarks and gluons. The imaginary part of a Pomeron exchange amplitude is usually related to multiple particle production in hadronic interactions. Let us first consider the eikonal formalism for quark scatterings via multiple Pomeron exchange. 
Denote the matrix element for a single Pomeron exchange as

$$
\begin{aligned}
\mathcal{M}_{\mathrm{el}}^{(1)} & =2 \pi i \delta\left(E_{i}-E_{f}\right) 2 \pi \sqrt{s} f_{1}(s, t), \\
f_{1}(s, t) & =\frac{-i}{2 \pi} \frac{g_{p}}{i \sqrt{s}} \bar{u}_{\sigma_{f}}\left(p_{f}\right) \not A(\mathbf{q}) u_{\sigma_{i}}\left(p_{i}\right) \\
& \equiv \frac{-i}{2 \pi} \frac{1}{i \sqrt{s}} T(s, \mathbf{q}),
\end{aligned}
$$

where $\sqrt{s} / 2=E_{i}=E_{f}$ and $g_{p}$ is a coupling constant between a quark and a Pomeron. The amplitude $f(s, t)$ is defined such that the differential cross section for elastic scatterings is given by

$$
\frac{d \sigma}{d t}=\pi|f(s, t)|^{2}
$$

The initial and final polarizations, $\sigma_{i}, \sigma_{f}$, should be averaged and summed over in the calculation of the cross sections. One can check that if $A(\mathbf{q})$ is replaced by a Debye screened Coulomb potential, $A(\mathbf{q})=g /\left(\mathbf{q}^{2}+\mu^{2}\right)$, and $g$ by the strong coupling constant, the above formula leads to $d \sigma / d t=4 \pi \alpha_{s}^{2} /\left(\mathbf{q}^{2}+\mu^{2}\right)^{2}$.

One can similarly write down the amplitude for double Pomeron exchange,

$$
\begin{gathered}
\mathcal{M}_{\mathrm{el}}^{(2)}=2 \pi i \delta\left(E_{i}-E_{f}\right)\left(-g_{p}^{2}\right) \quad \int \frac{d^{3} \ell}{(2 \pi)^{3}} \bar{u}_{\sigma_{f}}\left(p_{f}\right) \not A\left(\mathbf{p}_{f}-\ell\right) \frac{\ell}{\ell^{2}+i \epsilon} \not A\left(\ell-\mathbf{p}_{i}\right) u_{\sigma_{i}}\left(p_{i}\right) \\
e^{-i\left(\ell-\mathbf{p}_{i}\right) \cdot \mathbf{x}_{1}-i\left(\mathbf{p}_{f}-\ell\right) \cdot \mathbf{x}_{2}},
\end{gathered}
$$

where energy conservation at each coupling vertex sets the energy of the internal line to $\ell^{0}=\sqrt{s} / 2=E_{f}$. Amplitudes involving backscattering are suppressed at high energies, because of the limited momentum transfer that each the Pomeron can impart. If we assume that Pomerons do not overlap in space, the singularity in $A(\mathbf{q})$ can be neglected, and the integration over $\ell_{z}$ (with respect to the $\hat{z}$ direction of $\mathbf{x}_{21}=\mathbf{x}_{2}-\mathbf{x}_{1}=L \hat{z}+\mathbf{r}_{\perp}$ ) gives us

$$
\begin{aligned}
\mathcal{M}_{\mathrm{el}}^{(2)} & =2 \pi i \delta\left(E_{i}-E_{f}\right) \int \frac{d^{2} \ell_{\perp}}{(2 \pi)^{2}} \bar{u}_{\sigma_{f}}\left(p_{f}\right)\left(-g_{p}^{2}\right) \Gamma_{(2)} u_{\sigma_{i}}\left(p_{i}\right), \\
\Gamma_{(2)} & =\not A\left(\mathbf{p}_{f}-\mathbf{p}\right) \frac{\not p}{2 i p_{z}} \not A\left(\mathbf{p}-\mathbf{p}_{i}\right) e^{-i\left(\mathbf{p}-\mathbf{p}_{i}\right) \cdot \mathbf{x}_{1}-i\left(\mathbf{p}_{f}-\mathbf{p}\right) \cdot \mathbf{x}_{2}},
\end{aligned}
$$

where $p=\left(E_{f}, \sqrt{E_{f}^{2}-\ell_{\perp}^{2}}, \ell_{\perp}\right)$ is the four-momentum of the internal line. One can derive the classical Glauber multiple collision cross section from this amplitude by averaging and summing over the initial and final state ensemble of the target 27. In the limit of high-energy and small angle scattering, one can neglect the phase factor 
in the above equation and obtain the amplitude [as defined in Eq. (2)],

$$
f_{2}(s, t)=\frac{-i}{2 \pi} \int d^{2} b \frac{1}{2 !}[-\chi(\mathbf{b}, s)]^{2} e^{i \mathbf{q}_{\perp} \cdot \mathbf{b}},
$$

where $1 / 2$ ! comes from the different orderings of the target potentials and $\mathbf{q}_{\perp}=$ $\mathbf{p}_{f \perp}-\mathbf{p}_{i \perp}$ is the total transverse momentum transfer from the multiple scatterings. The eikonal function $\chi_{\sigma_{1}, \sigma_{2}}(\mathbf{b}, s)$ is defined as the Fourier transform of the single scattering amplitude (besides a factor $i / 2 \pi$ ),

$$
\begin{aligned}
\chi(\mathbf{b}, s) & \equiv \frac{i}{\sqrt{s}} \int \frac{d^{2} q_{\perp}}{(2 \pi)^{2}} e^{-i \mathbf{q}_{\perp} \cdot \mathbf{b}} T\left(s, \mathbf{q}_{\perp}\right) \\
& =\frac{i}{4 \pi \sqrt{s}} \int_{-\infty}^{0} d t J_{0}(b \sqrt{-t}) T(s, t)
\end{aligned}
$$

where $t=-\mathbf{q}_{\perp}^{2}$ and $J_{0}$ is the zeroth order Bessel function. In the definition of the product of eikonal functions, summation over the polarizations of the intermediate lines is implied. If we are only interested in unpolarized collisions, we can regard $T\left(s, \mathbf{q}_{\perp}\right)$ as a scalar in the following. One can generalize the double scattering amplitude to multiple scatterings and sum them together to get the total amplitude,

$$
\begin{aligned}
f(s, t)=\sum_{n=1}^{\infty} f_{n}(s, t) & =\frac{-i}{2 \pi} \int d^{2} b \sum_{n} \frac{1}{n !}[-\chi(\mathbf{b}, s)]^{n} e^{i \mathbf{q}_{\perp} \cdot \mathbf{b}} \\
& =\frac{i}{2 \pi} \int d^{2} b\left[1-e^{-\chi(\mathbf{b}, s)}\right] e^{i \mathbf{q}_{\perp} \cdot \mathbf{b}} .
\end{aligned}
$$

This is the total elastic quark scattering amplitude in the eikonal formalism. This amplitude can also be derived from the scattering theory in quantum mechanics [39], where the eikonal function is related to the potential experienced by the scattering particle.

One can consider a $p p$ interaction as multiple quark-quark scatterings and derive a similar formula for the total amplitude of an elastic $p p$ collision. The effective eikonal function for $p p$ collisions now should be given by

$$
\chi(\mathbf{b}, s)=\frac{i}{\sqrt{s}} \int \frac{d^{2} q_{\perp}}{(2 \pi)^{2}} e^{-i \mathbf{q}_{\perp} \cdot \mathbf{b}} t\left(\mathbf{q}_{\perp}, s\right) t\left(-\mathbf{q}_{\perp}, s\right) T\left(s, \mathbf{q}_{\perp}\right),
$$

where $t\left(\mathbf{q}_{\perp}, s\right)$ is the Fourier transform of the quark density distribution inside a nucleon and $T\left(s, \mathbf{q}_{\perp}\right)$ is the amplitude of a quark-quark scattering with one Pomeron exchange. Here we neglected the contribution from Reggeon exchanges, so that the amplitude $T\left(s, \mathbf{q}_{\perp}\right)$ is purely imaginary [28] (or the eikonal function is real); thus 
the elastic amplitude of $p p$ collisions, Eq.( 9), is also purely imaginary. Experimental measurements of high-energy $p p$ or $p \bar{p}$ collisions indeed find the real part of the elastic amplitude to be small [40].

With the above elastic amplitude, the differential elastic cross section for $p p$ collisions is given by Eq. (4). Using the identity

$$
\int_{-\infty}^{0} d t J_{0}(b \sqrt{-t}) J_{0}\left(b^{\prime} \sqrt{-t}\right)=\frac{2}{b} \delta\left(b-b^{\prime}\right)
$$

one can obtain the elastic cross section,

$$
\sigma_{\mathrm{el}}=\int d^{2} b\left[1-e^{-\chi(b, s)}\right]^{2}
$$

where we assumed that the imaginary part of the eikonal function is negligible. Using the optical theorem, one can also get the total and inelastic cross sections of $p p$ collisions [35, 41,

$$
\begin{aligned}
\sigma_{\text {tot }} & =4 \pi \operatorname{Im} f(s, t=0)=2 \int d^{2} b\left[1-e^{-\chi(b, s)}\right], \\
\sigma_{\text {in }} & =\sigma_{\text {tot }}-\sigma_{\text {el }}=\int d^{2} b\left[1-e^{-2 \chi(b, s)}\right] .
\end{aligned}
$$

The approximation of a small imaginary (real) part of the eikonal function (elastic amplitude) in Eq. (10) also leads us to assume that the quark-quark scattering amplitude $T\left(s, \mathbf{q}_{\perp}\right)$ with one Pomeron exchange is also purely imaginary [cf. Eqs. (9) and (10)]. According to Eq. (3) and the optical theorem, $T\left(s, \mathbf{q}_{\perp}\right)$ should be related to the total inclusive cross section of quark-quark scatterings,

$$
T(s, \mathbf{0})=-i \frac{\sqrt{s}}{2} \sigma_{\text {incl }}(s) .
$$

Assuming the dominance of small angle contributions in Eq. (10), we have then the eikonal function for $p p$ collisions,

$$
\chi(b, s) \approx \frac{1}{2} \sigma_{\text {incl }}(s) T_{N}(b, s)
$$

where $T_{N}(b, s)$ is the overlap function of two nucleons,

$$
T_{N}(b, s)=\int d^{2} b^{\prime} t\left(\mathbf{b}^{\prime}, s\right) t\left(\mathbf{b}-\mathbf{b}^{\prime}, s\right)
$$

which is normalized to one, $\int d^{2} b T_{N}(b, s)=1$. 


\subsection{Cross Sections: Soft vs. Hard}

To calculate the total inclusive cross section of quark-quark scatterings, $\sigma_{\text {incl }}(s)$, one needs to know the Pomeron structure and its coupling to quarks and gluons. In a simple model, one can regard a Pomeron as a double gluon exchange with a ladder structure as illustrated in Fig. 1(a). In general, most of the loops inside the ladder have soft momenta and thus cannot be calculated via pQCD. We consider this nonperturbative contribution to the inclusive cross section as $\sigma_{\text {soft }}(s)$. As the colliding energy $\sqrt{s}$ increases, contributions from large momentum, $p_{T}>p_{0}$, to the loop integral also increase. As illustrated in Fig. 1(b), the cutting diagram of such a Pomeron exchange which contains at least one hard loop corresponds to hard scatterings in $p p$ collisions. If one assumes that the loop momenta are ordered so that they increase toward the hard loop, then the rest of the cut-Pomeron beside the hard loop can be resummed to give the quark or gluon (parton) distributions of a nucleon and their Gribov-Lipatov-Altarelli-Parisi (GLAP) [42] evolution. The corresponding contribution to the inclusive cross section can then be identified as the inclusive jet cross section [13],

$$
\begin{aligned}
\sigma_{\text {jet }} & =\int_{p_{0}^{2}}^{s / 4} d p_{T}^{2} d y_{1} d y_{2} \frac{1}{2} \frac{d \sigma_{\text {jet }}}{d p_{T}^{2} d y_{1} d y_{2}} \\
\frac{d \sigma_{\text {jet }}}{d p_{T}^{2} d y_{1} d y_{2}} & =K \sum_{a, b} x_{1} f_{a}\left(x_{1}, p_{T}^{2}\right) x_{2} f_{b}\left(x_{2}, p_{T}^{2}\right) \frac{d \sigma^{a b}(\hat{s}, \hat{t}, \hat{u})}{d \hat{t}}
\end{aligned}
$$

where the summation runs over all parton species, $y_{1}$ and $y_{2}$ are the rapidities of the scattered partons, and $x_{1}$ and $x_{2}$ are the light-cone momentum fractions carried by the initial partons. These variables are related by $x_{1}=x_{T}\left(e^{y_{1}}+e^{y_{2}}\right) / 2, x_{2}=$ $x_{T}\left(e^{-y_{1}}+e^{-y_{2}}\right) / 2, x_{T}=2 p_{T} / \sqrt{s}$. The $f_{a}\left(x, Q^{2}\right)$ in Eq. (19) are the parton distribution functions. The pQCD cross sections, $d \sigma_{a b}$, depend on the subprocess variables $\hat{s}=$ $x_{1} x_{2} s, \hat{t}=-p_{T}^{2}\left(1+\exp \left(y_{2}-y_{1}\right)\right)$, and $\hat{u}=-p_{T}^{2}\left(1+\exp \left(y_{1}-y_{2}\right)\right)$. A factor $K \approx 2$ is included to correct the lowest order pQCD rates for next to leading order effects [44, 45].

At small $x \sim p_{T} / \sqrt{s}$, the momentum in the ladder diagram might not be ordered anymore and there might be more than one hard loop inside the ladder. However, calculations [46] have shown that such contributions are still small at present and future collider energies. If we neglect contributions from multiple hard loops , the total inclusive quark-quark cross section can be written as

$$
\sigma_{\text {incl }}=\sigma_{\text {soft }}(s)+\sigma_{\text {jet }}(s) .
$$


(a)

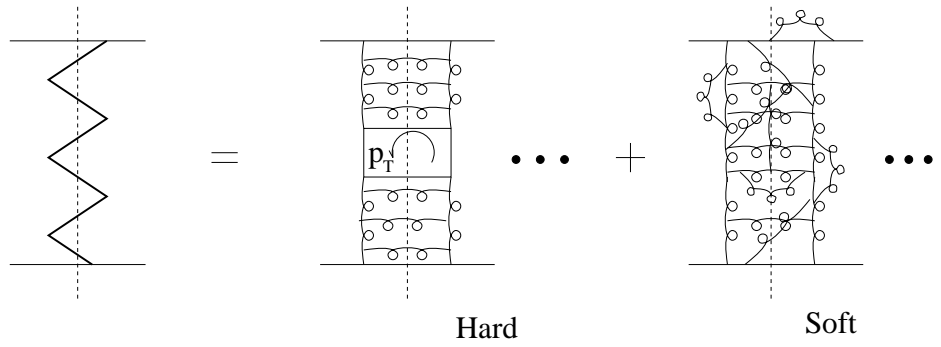

(b)

Figure 1: (a) A Pomeron can be divided into the ladder diagrams of a hard part, in which at least one loop momentum $p_{T}$ is large, and a corresponding soft part. (b) The hard part of a cut-Pomeron can be factorized into parton distributions and hard scattering matrix elements.

where $\sigma_{\text {soft }}$ represents the contribution from the cut-Pomeron in which the transverse momenta of the loops are limited to $p_{T}<p_{0}$, and therefore is not calculable via pQCD. However, as we can see from its definition, $\sigma_{\text {soft }}$ represents processes involving many soft partons in the final state. It cannot be modeled by a simple two parton elastic scattering. Since there is no ordering in $p_{T}$, interference becomes important in soft particle production, which can be modeled by, e.g., a string model.

Given the above decomposition of the total inclusive cross section and the eikonal function in Eq. (16), we can rewrite the inelastic cross section for $p p$ collisions, Eq. (14), as

$$
\begin{aligned}
\sigma_{\mathrm{in}} & =\int d^{2} b\left[1-e^{-\left(\sigma_{\mathrm{soft}}+\sigma_{\mathrm{jet}}\right) T_{N}(b, s)}\right] \equiv \int d^{2} b \sum_{j=0}^{\infty} g_{j}(b) ; \\
g_{j}(b) & =\frac{\left[\sigma_{\mathrm{jet}} T_{N}(b, s)\right]^{j}}{j !} e^{-\sigma_{\mathrm{jet}} T_{N}(b, s)}, \quad j \geq 1 ; \\
g_{0}(b) & =\left[1-e^{-\sigma_{\mathrm{soft}} T_{N}(b, s)}\right] e^{-\sigma_{\mathrm{jet}} T_{N}(b, s)} .
\end{aligned}
$$

where $g_{j}(b)$ can be considered as the probability for $j$ hard scatterings [35, 47] among inelastic collisions at fixed impact paramenter $b$. Note that a jet in our terminology refers to a large $p_{T}$ hard scattering. The average number of jets with $p_{T} \geq p_{0}$ 
in $p p$ collisions is thus $\left\langle n_{\text {jet }}\right\rangle=\sigma_{\text {jet }} / \sigma_{\text {in }}$. The above probabilistic interpretation of multiple hard scatterings depends on our assumption that multiple cut-Pomerons are independent from each other. This holds as long as the average number of hard scatterings is not too large. Given an interaction transverse area, $\sim \pi / p_{0}^{2}$, for processes with $p_{T} \sim p_{0}$, independence requires that the total interaction area is less than $\pi R_{N}^{2}$, where $R_{N} \approx 0.85 \mathrm{fm}$ is the nucleon radius; i.e.,

$$
\sigma_{\text {jet }} \lesssim\left(p_{0} R_{N}\right)^{2} \sigma_{\text {in }} \equiv \sigma_{\max }
$$

For $p_{0} \gtrsim 2 \mathrm{GeV} / c$, and $\sigma_{\text {in }} \approx 40 \mathrm{mb}$, the right hand side is $\sigma_{\max } \approx 3$ barns, and thus the independent approximation should hold up to the highest energies foreseen. For nuclear collisions the total number of jets is given by

$$
N_{\text {jet }}^{A A}=T_{A A}(b) \sigma_{\text {jet }}
$$

where $T_{A A}(b)$ is the nuclear overlap function at an impact parameter $b$. For $b=0$, $T_{A A} \approx A^{2} / \pi R_{A}^{2}$, and multiple mini-jets may be independent as long as

$$
\sigma_{\text {jet }} \lesssim\left(p_{0} R_{A}\right)^{2} \pi R_{A}^{2} / A^{2} \approx 2 \sigma_{\max } / A^{2 / 3}
$$

For $A=197$ the right hand side is $180 \mathrm{mb}$, and thus independence should apply up to LHC energies (see Fig. 2 below). On the other hand, nuclear shadowing of the initial structure functions will reduce the jet cross section so that independence should be valid beyond LHC energies.

For $p_{0}>1 \mathrm{GeV}, \sigma_{\text {jet }}(s)$ is found to be very small when $\sqrt{s} \lesssim 20 \mathrm{GeV}$ and only the soft component is important. The low-energy data of diffractive nucleonnucleon scatterings exhibit a number of geometrical scaling properties 48 in the range $10<\sqrt{s}<100 \mathrm{GeV}$, e.g., $\sigma_{\mathrm{el}} / \sigma_{\mathrm{tot}} \cong 0.175$, and $B / \sigma_{\text {tot }} \cong 0.3$, where $B$ is the slope of the diffractive peak of the differential elastic cross section. This suggests a geometrical scaling form 49] for the eikonal function at low energies; i.e., it is only a function of $\xi=b / b_{0}(s)$, with $\pi b_{0}^{2}(s) \equiv \sigma_{\text {soft }}(s) / 2$ providing a measure of the geometrical size of the nucleon. We further assume that the nucleon overlap function is given by the Fourier transform of a dipole form factor so that,

$$
\begin{aligned}
T_{N}(b, s) & =\frac{\chi_{0}(\xi)}{\sigma_{\text {soft }}(s)} \\
\chi_{0}(\xi) & =\frac{\mu_{0}^{2}}{48}\left(\mu_{0} \xi\right)^{3} K_{3}\left(\mu_{0} \xi\right), \quad \xi=b / b_{0}(s)
\end{aligned}
$$




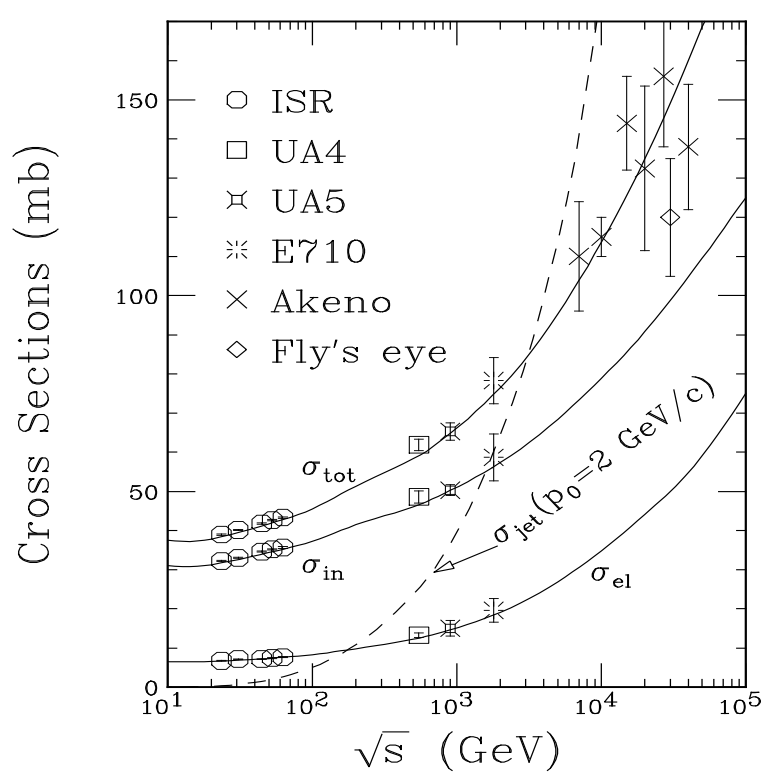

Figure 2: The total, inelastic, and elastic cross sections of $p p$ and $p \bar{p}$ collisions calculated by HIJING (solid lines) as compared to the data 448, 50, 51, 52, 53, 54]. The dashed line is the total inclusive jet cross section with $p_{T} \geq p_{0}=2 \mathrm{GeV} / c$.

where $\mu_{0}=3.9$. With this assumption, the eikonal function can be written as,

$$
\chi(b, s) \equiv \chi(\xi, s)=\chi_{0}(\xi)\left[1+\sigma_{\text {jet }}(s) / \sigma_{\text {soft }}(s)\right]
$$

This form ensures that geometrical scaling[49] is recovered at low energies when $\sigma_{\text {jet }} \ll$ $\sigma_{\text {soft }}$.

Choosing $p_{0} \simeq 2 \mathrm{GeV} / c$ and assuming a constant value of $\sigma_{\text {soft }}=57 \mathrm{mb}$ at high energies, the calculated cross sections and the multiplicity distributions in $p p$ and $p \bar{p}$ collisions agree well with experiments[35]. This is the model adopted in HIJING [19] to simulate multiple jet production at the level of nucleon-nucleon collisions. In Fig. 2, the calculated total, inelastic and elastic cross sections of $p p$ or $p \bar{p}$ collisions are shown as functions of $\sqrt{s}$ (solid line) together with experimental data 48, 50, 51, 52, 53, 54. The dashed line corresponds to the inclusive jet cross section. The calculated cross sections agree well with experiments from ISR to Tevatron and cosmic-ray energies. We note that the total inclusive jet cross section increases much faster than the inelastic cross section as a function of $\sqrt{s}$, leading to an increase of the average number of minijets, $\sigma_{\text {jet }} / \sigma_{\text {in }}$, with energy.

We emphasize that the value of $p_{0}=2 \mathrm{GeV}$ used in the above calculation is only a phenomenological parameter. In order for the model to have some predictive power, $p_{0}$ should not depend on $\sqrt{s}$. However, its value is subject to considerable 
controversy [33]. The problem arises from the fact that there is not a clear boundary specified by $p_{0}$ between soft and hard processes, as shown in Eqs. (18) and (20). If we believe that pQCD can be reliably applied to calculate hard contributions to the total inclusive cross section up to $p_{0}$, then the rest must be modeled by a phenomenological $\sigma_{\text {soft }}$. Clearly, $\sigma_{\text {soft }}(s)$ must depend on the choice of $p_{0}$. With a smaller (larger) $p_{0}$, more (less) contributions are included as hard collisions. Hence $\sigma_{\text {soft }}(s)$ would be smaller (larger). Obviously many choices of $p_{0}$ and $\sigma_{\text {soft }}(s)$ can give the same total cross section $\sigma_{\text {tot }}(s)$. The only restriction is that the sum $\sigma_{\text {soft }}(s)+\sigma_{\text {jet }}(s)$ must have the right value to give the right energy dependence of the total cross section $\sigma_{\text {tot }}(s)$. Since $\sigma_{\text {jet }}(s)$ increases with decreasing $p_{0}$ and $\sigma_{\text {soft }}(s)$ is non-negative, $p_{0}$ must be bounded from below by the experimental data on the total cross section $\sigma_{\text {tot }}(s)$. We find that this lower limit in our model is $p_{0}=1.2 \mathrm{GeV}$ with the given parton distribution functions. For $p_{0}$ smaller than $1.2 \mathrm{GeV}$, the inclusive jet cross section at high energies is overestimated and the resultant $\sigma_{\text {tot }}(s)$ can never fit the data. In addition, one must keep in mind that the lowest $p_{0}$ is also bounded by the relevant $Q_{0}=p_{0}$ in the evolution of the parton distribution functions.

In HIJING, the parton distribution functions are taken to be the Duke-Owens 555 parametrization set 1 with $Q_{0}=2 \mathrm{GeV}$. The latest version of this parameterization (DO1.1) for $f_{a}\left(x, Q^{2}\right)$ is adequate through RHIC energies. However, at higher energies, more updated distributions, such as GRV [56] or MRSD-' [57] should be used. These distributions, constrained by the most recent HERA data [58] in deeply inelastic ep collisions, are more divergent at small $x$ and give rise to larger minijet cross sections, especially at the LHC energy [59]. Using these distributions, one cannot fit $\sigma_{\text {tot }}(s)$ at high energies with fixed $p_{0}$. As has been discussed in Ref. 46], this is due to multiple hard loops in one Pomeron exchange. The divergent behavior of the distribution functions is usually related to the fact that the hard loops are not ordered in $p_{T}$, which gives rise to multiple jet pair production per cut-Pomeron. Thus the inclusive jet cross section in the eikonal function must be normalized by the average number of such jet pairs 60.

\subsection{Modeling the Soft Interactions}

From Fig. 1(a), we can see that a cut-Pomeron always produces many soft partons with small transverse momenta no matter whether there is a hard process present or not. Unlike partons from initial and final state radiations associated with a hard scattering, these soft partons are not ordered in transverse momentum and coherence is extremely important, which could virtually produce a coherent color field. Therefore, soft interactions may not be modeled simply by regularized parton-parton elastic scatterings with small transverse momentum transfers. On the other hand, 
the color field could also be screened significantly later by the interaction with the hard or semihard partons from the hard collisions 61] in heavy-ion collisions. The screening effect will decrease the final particle production from the color field.

As demonstrated in three-jet events in $e^{+} e^{-}$annihilations, the color interference effects can be approximated fairly well by a string model 62. In HIJING we adopted a variant of the multiple string phenomenological model for the soft interaction as developed in Refs. [13, 14, 16]. Those soft interactions must naturally involve small $p_{T}$ transfer to the constituent quarks, as well as induced soft gluon radiation which can be modeled by introducing kinks in the strings. The produced partons are treated either as hard kinks (for gluons) on the string or the end points (for quarks) of another string. The strings are assumed to decay independently via quark-antiquark creation using, in our case, the Lund JETSET7.2 63 fragmentation routine to describe the hadronization.

The string excitation is achieved by a collective momentum transfer $P=$ $\left(P^{+}, P^{-}, \mathbf{p}_{T}\right)$ between the hadrons. Given initial light-cone momenta

$$
p_{1}=\left(p_{1}^{+}, \frac{m_{1}^{2}}{p_{1}^{+}}, \mathbf{0}_{T}\right), \quad p_{2}=\left(\frac{m_{2}^{2}}{p_{2}^{-}}, p_{2}^{-}, \mathbf{0}_{T}\right)
$$

with $\left(p_{1}^{+}+m_{2}^{2} / p_{2}^{-}\right)\left(p_{2}^{-}+m_{1}^{2} / p_{1}^{+}\right) \equiv s$, the final momenta of the strings are assumed to be

$$
p_{1}^{\prime}=\left(p_{1}^{+}-P^{+}, \frac{m_{1}^{2}}{p_{1}^{+}}+P^{-}, \mathbf{p}_{T}\right), \quad p_{2}^{\prime}=\left(\frac{m_{2}^{2}}{p_{2}^{-}}+P^{+}, p_{2}^{-}-P^{-},-\mathbf{p}_{T}\right) .
$$

The remarkable feature of soft interactions is that low transverse momentum exchange processes with $p_{T} \lesssim 1 \mathrm{GeV} / c$ can result in large effective light-cone momentum exchanges [13], giving rise to two excited strings with large invariant mass. Defining

$$
P^{+}=x_{+} \sqrt{s}-\frac{m_{2}^{2}}{p_{2}^{-}}, \quad P^{-}=x_{-} \sqrt{s}-\frac{m_{1}^{2}}{p_{1}^{+}}
$$

the excited masses of the two strings will be

$$
M_{1}^{2}=x_{-}\left(1-x_{+}\right) s-p_{T}^{2}, \quad M_{2}^{2}=x_{+}\left(1-x_{-}\right) s-p_{T}^{2},
$$

respectively. In HIJING, we require that the excited string mass must exceed a minimum value $M_{\text {cut }}=1.5 \mathrm{GeV}$, and therefore the kinematically allowed region of $x^{ \pm}$is restricted to

$$
x_{-}\left(1-x_{+}\right) \geq M_{\text {Tcut } 1}^{2} / s, \quad x_{+}\left(1-x_{-}\right) \geq M_{\text {Tcut } 2}^{2} / s,
$$


where $M_{T \text { cut } 1}^{2}=M_{\text {cut }}^{2}+p_{T}^{2}, M_{T \text { cut } 2}^{2}=M_{\text {cut }}^{2}+p_{T}^{2}$. Only collisions with

$$
s \geq s_{\min }=\left(M_{\text {Tcut } 1}+M_{T \text { cut } 2}\right)^{2}
$$

are allowed to form excited strings. Eq. (35) also determines the maximum $p_{T}$ that the strings can obtain from the soft interactions. In events with both hard and soft processes, two strings are still assumed to form but with a kinetic boundary reduced by the hard scatterings.

In HIJING, the probability for light-cone momentum transfer is assumed to be

$$
P\left(x_{ \pm}\right)=\frac{\left(1.0-x_{ \pm}\right)^{1.5}}{\left(x_{ \pm}^{2}+c^{2} / s\right)^{1 / 4}}
$$

for nucleons and

$$
P\left(x_{ \pm}\right)=\frac{1}{\left(x_{ \pm}^{2}+c^{2} / s\right)^{1 / 4}\left[\left(1-x_{ \pm}\right)^{2}+c^{2} / s\right]^{1 / 4}}
$$

for mesons, with $c=0.1 \mathrm{GeV}$, along the lines of the DPM model [14]. Soft gluon bremsstrahlung processes with $p_{T}<p_{0}$ are also introduced as kinks along the excited string. In addition, HIJING also includes an extra low $p_{T}<p_{0}$ transfer to the constituent quarks and diquarks at the string end points in soft interactions. This effect is important at low energies, $E_{\text {lab }} \sim 20 \mathrm{GeV}$, to account for the high $p_{T}$ tails of the pion and proton distributions [19].

I emphasize that the low $p_{T}$ algorithm used in HIJING is a phenomenological model needed to incorporate non-perturbative aspects of beam jet physics. Many variants of soft dynamics can be envisioned, but none can be rigorously defended from fundamental QCD. One of the attractive aspects of going to the highest possible collider energies is that the theoretical uncertainties due to soft dynamics are reduced, as more and more of the dynamics becomes dominated by calculable semi-hard and hard QCD processes.

\subsection{Minijets and Transverse Flow}

To summarize the consistency of HIJING calculations with the available experimental data, I show in Fig. 3 the inclusive spectra of charged particles in $p p$ and $p \bar{p}$ over a wide energy range, $\sqrt{s}=50-1800 \mathrm{GeV}$. We see that the model accounts well for the energy dependence of not only the transverse momentum distribution, but also the rapidity distribution, as well as the multiparticle fluctuations. This overall quantitative understanding of multiparticle observables in hadronic interactions, especially the magnitude and energy dependence of the conspicuous power-law tail of the $p_{T}$ spec- 


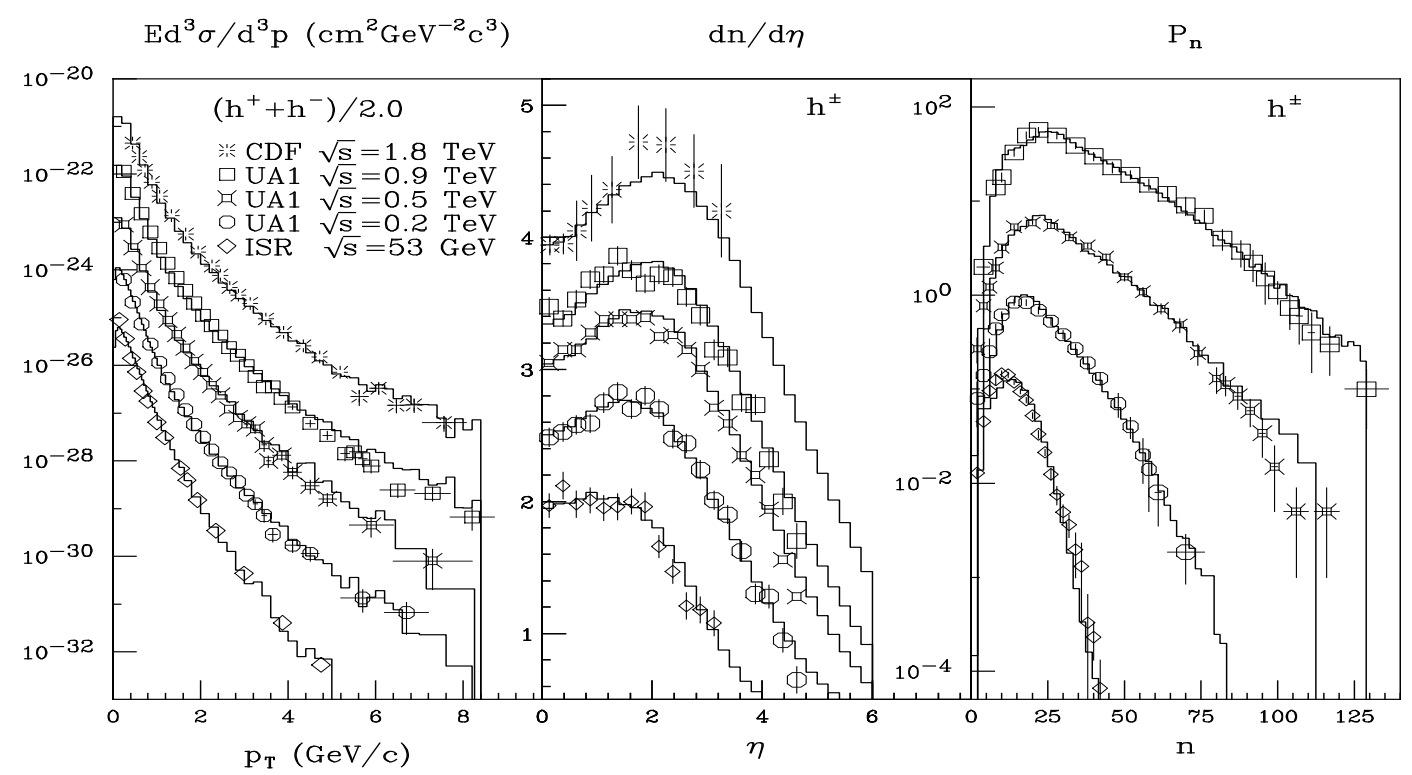

Figure 3: Data on charged particle inclusive $p_{T}$ spectra $E d^{3} \sigma / d^{3} p$ 64, 65, 66, pseudorapidity distributions $d n / d \eta$ [67, 68], and multiplicity distributions [69, 70, 71] $P_{n}$ in $p p$ and $p \bar{p}$ collisions compared to HIJING calculations at different energies $\sqrt{s}$. The transverse momentum spectra and multiplicity distributions have been displaced for clarity by extra factors of 10 relative to the absolutely normalized data at $\sqrt{s}=53$ $\mathrm{GeV}$.

trum characteristic of pQCD, strongly supports the importance of minijets physics at collider energies. To demonstrate the onset of particle production with minijet production, I plot in Fig. $⿴$ t the energy dependence of the central rapidity density of produced charged particles. I also show the contribution from purely soft interactions. Since the central rapidity density from soft string fragmentations is almost constant as a function of the colliding energy, the increased $d n_{\mathrm{ch}} / d \eta$ mainly comes from the hadronization of jets at high energies. The correlation between the central rapidity density and minijets is very clear when $d n_{\mathrm{ch}} / d \eta$ is compared with the average number of minijets, $\left\langle n_{\text {jet }}\right\rangle=\sigma_{\text {jet }} / \sigma_{\text {in }}$, as functions of $\sqrt{s}$.

Beside providing overall agreement with experimental data on multiparticle distribution and spectra [17, 84], minijet production will also influence correlations between observed quantities, some of which could be mistakenly attributed to QGP and other collective phenomena. One typical example is the multiplicity and mass dependence of the average transverse momentum observed in $p \bar{p}$ collisions at Tevatron Collider energy [75]. In hydrodynamic models, transverse collective flow is usually generated from the expansion of the thermalized dense system [76]. Since all hadrons have the same flow velocity, heavy particles tend to have larger transverse momentum 


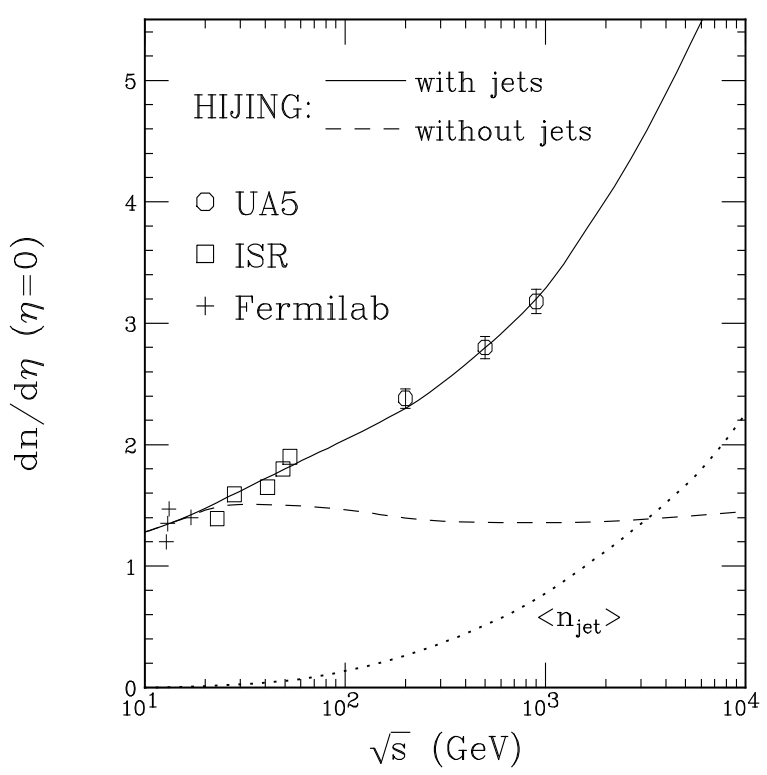

Figure 4: $\quad d n_{\mathrm{ch}} / d \eta(\eta=0)$ in inelastic $p p$ and $p \bar{p}$ collisions as a function of $\sqrt{s}$. The solid line is the HIJING calculation compared to the data [67, 72, 73]. The dashed line is for events without jet production in HIJING simulations. The dotted line is the calculated average number of jets, $\left\langle n_{\text {jet }}\right\rangle=\sigma_{\text {jet }} / \sigma_{\text {in }}$.

when they finally freeze out. If the average transverse momentum is plotted against the total multiplicity, it is anticipated that for heavy particles it will be larger and the increase with the multiplicity will be faster than for light ones. Quite surprisingly, experiments on $p \bar{p}$ collisions at Tevatron Collider energy have recently reported observation of just this effect [75]. Lévai and Müller [77] have studied this reaction in a linearized transport theory. They found that there is no time for the baryons to equilibrate with the pions during the expansion of a hadronic fireball. They therefore suggested that a more novel explanation could be required to account for the apparent similarity of the flow velocities of the mesons and baryons. They noted that these observations could be understood if an equilibrated quark-gluon plasma (QGP) were formed in these collisions 78 .

However, as also noted by Lévai and Müller, the common transverse flow of hadrons may also arise accidentally from the fragmentation of minijets. This is indeed what has been found with HIJING calculations [79]. Shown in Fig. 5 is the HIJING result of the correlation between $\left\langle p_{T}\right\rangle$ and the total charged multiplicity $n_{\mathrm{ch}}$ for pions, kaons, and antiprotons (from bottom to top) as solid lines together with the data at Tevatron energy. The average multiplicity density $\left\langle d n_{\mathrm{ch}} / d \eta\right\rangle$ is calculated as $n_{\mathrm{ch}}(|\eta|<\Delta \eta)$ divided by $2 \Delta \eta$. The average $p_{T}$ is obtained by applying the same procedure as used in the experiment [75] in which the $p_{T}$ distributions are first fitted 


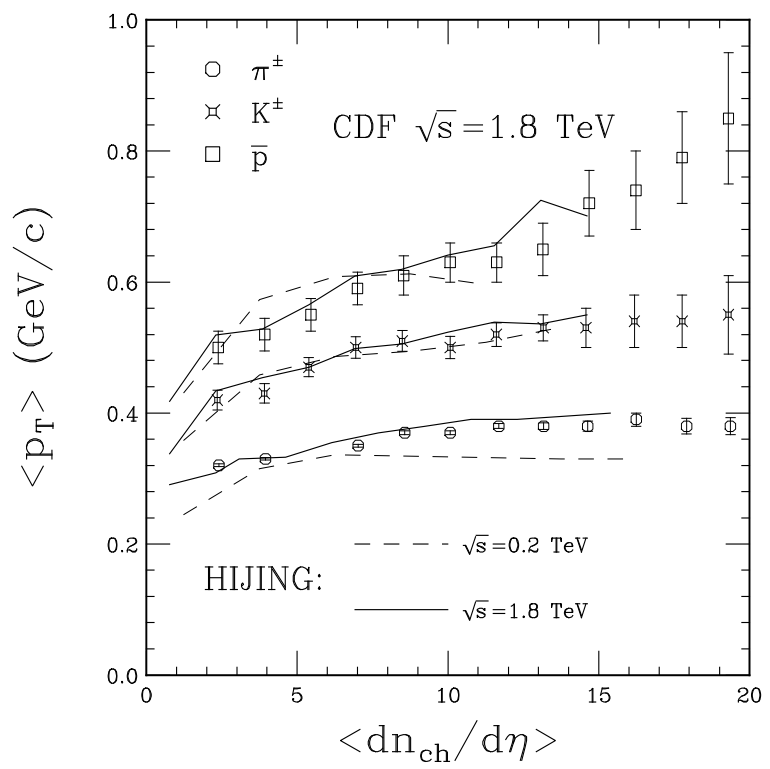

Figure 5: $\left\langle p_{T}\right\rangle$ of pions, kaons, and anti-protons (from bottom to top) in $-0.36<$ $\eta<1.0$ versus the average charged multiplicity density $d n_{\mathrm{ch}} / d \eta$ in $|\eta|<3.25$. The histograms are HIJING results and points are the data [75]. The dashed lines are for $p p$ at $\sqrt{s}=200 \mathrm{GeV}$.

with parametrizations [power law $a\left(p_{T}+b\right)^{-n}$ for pions and exponential $\beta \exp \left(-\alpha p_{T}\right)$ for kaons and antiprotons] and then the fitted parameters are used to calculate $\left\langle p_{T}\right\rangle$ in the restricted range $0<p_{T}<1.5 \mathrm{GeV} / c$. Apparently, the data are well accounted for by our calculation. Shown as dashed lines in Fig. 5 are the calculated results for $p p$ collisions at RHIC energy, $\sqrt{s}=200 \mathrm{GeV}$. They are similar to the results at $\sqrt{s}=1.8$ $\mathrm{TeV}$, except that pions have a lower saturated value of $\left\langle p_{T}\right\rangle$ at RHIC energy. Since pions are the dominant produced particles, the high multiplicity $\left\langle p_{T}\right\rangle$ for all charged hadrons at $\sqrt{s}=200 \mathrm{GeV}$ is smaller than at $\sqrt{s}=1.8 \mathrm{TeV}$.

In a model with multiple parton production, it is easy to understand why $\left\langle p_{T}\right\rangle$ increases with $n_{\mathrm{ch}}$. If we decompose the multiplicity distribution into different contributions from events with different number of minijets as shown in Fig. 6, we find that large multiplicity events are dominated by multiple minijet production while low multiplicity events are dominated by those of no jet production. The average transverse momentum in events with multiple minijets is certainly larger than those without, thus leading to the increase of $\left\langle p_{T}\right\rangle$ with $n_{\mathrm{ch}}$. In order to understand the different behavior of the correlations between $\left\langle p_{T}\right\rangle$ and $n_{\mathrm{ch}}$ for different particles, we recall that the jet fragmentation functions for heavy hadrons tend to be harder than for light hadrons, as measured in $e^{+} e^{-}$annihilation experiments 80. Therefore, heavy hadrons from jet fragmentation carry larger transverse momenta than light hadrons in 


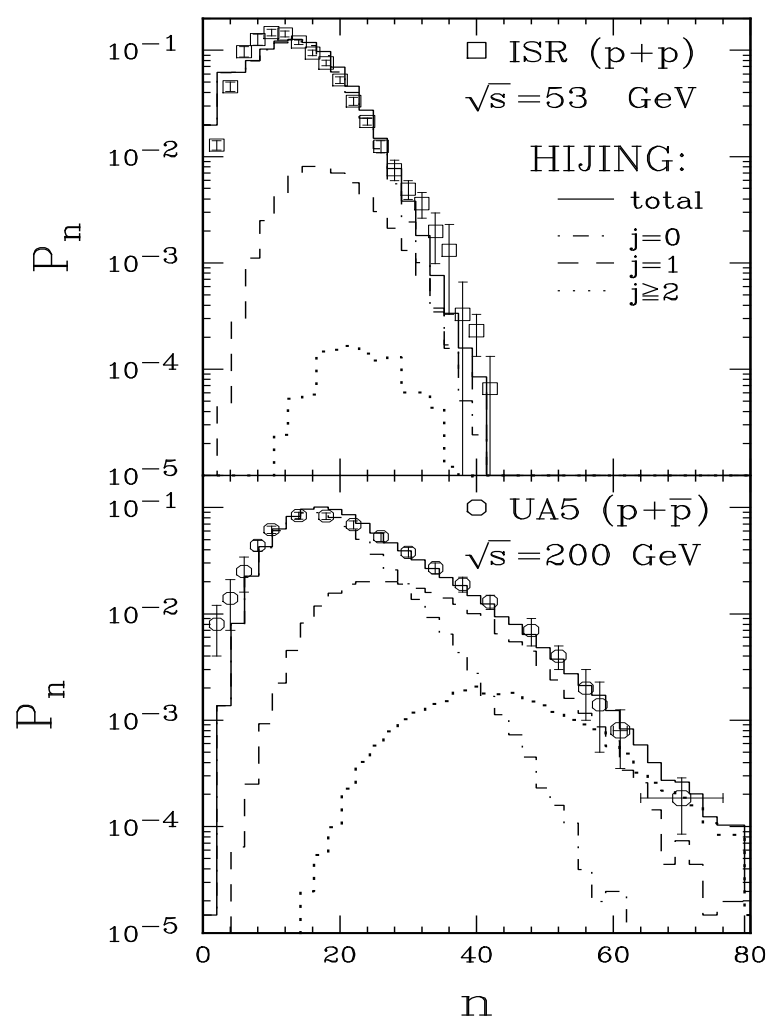

Figure 6: Charged multiplicity distributions in NSD $p p$ at $\sqrt{s}=53 \mathrm{GeV}$ and $p \bar{p}$ collisions at $\sqrt{s}=200 \mathrm{GeV}$. The data are from Refs. [69, 70]. The solid histograms are from the HIJING calculation with contributions from events with $j=0$ (dotdashed histograms), $j=1$ (dashed histograms), and $j \geq 2$ (dotted histograms) jet production.

$p p$ or $p \bar{p}$ collisions. This leads naturally to larger slopes of the $\left\langle p_{T}\right\rangle$ vs $n_{\mathrm{ch}}$ correlation for heavier particles. In other words, the fragmentation of minijets can mimic the transverse "flow" effect giving the resultant appearance of collective behavior.

Now one may ask: if totally different models [77, 81] can both describe the data, which one has the true underlying dynamics and how can they be distinguished from each other? To answer these questions, one has to turn to the properties of jet fragmentation.

\subsection{Resolving Minijets in Hadronic Interactions}

Jets in hadronic interactions, as defined earlier, are produced by large $p_{T}$ parton scatterings in pQCD. Experimentally, jets are identified as hadronic clusters whose transverse energy $E_{T}$ can be reconstructed from a calorimetrical study of the events [83]. However, this cluster-finding method becomes questionable for small and even 
intermediate $E_{T}$ values due to the background of fluctuations 84] from the underlying soft interactions. It has therefore been very difficult to resolve minijets with $p_{T} \gtrsim 2$ $\mathrm{GeV} / c$ from the underlying soft background.

Although minijets with $p_{T} \gtrsim 2 \mathrm{GeV} / c$ are difficult to resolve as distinct jets from the background, their effects even in minimum biased events, as we have demonstrated, can explain many aspects of hadronic collisions and the associated multiparticle production [33]. I have proposed [82] that the $p_{T}$ dependence of the two-particle correlation function can be utilized to study the minijet content in the minimum biased events of hadronic interactions. Because particles from jet fragmentation tend to cluster in phase space, two-particle correlations must be enhanced due to minijet production. Especially for two-particle correlations in the azimuthal angle $\phi$, contributions from back-to-back minijets should be strongly peaked in both forward $(\Delta \phi=0)$ and backward $(\Delta \phi=\pi)$ directions. If we calculate the same correlations, but for some selected particles whose transverse momenta are larger than a certain $p_{T}$ cut, the two peaks should be more prominent because these particles are more likely to come from minijets. On the other hand, particles from soft production or an expanding quark gluon plasma are isotropical in the transverse plane and would only have some nominal correlation in the backward direction due to momentum conservation. Therefore, the experimental measurement of two-particle correlation functions and their $p_{T}$ dependence, especially in $p \bar{p}$ collisions at the Fermilab Tevatron energy where multiplicity and mass dependence of $\left\langle p_{T}\right\rangle$ were first observed, is essential to end the present controversy over whether the phenomenon is due to minijets, or string interaction 81] or the formation of a quark gluon plasma [77.

It is well known that particles from high $p_{T}$ jets are very concentrated in both directions of the back-to-back jets. The widths of these high $p_{T}$ jet profiles are about 1 in both pseudorapidity $\eta$ and azimuthal angle $\phi$ 85. Minijets, though with smaller $p_{T}$, should have similar properties. Since particles with $p_{T}>p_{T \text { cut }}$ are more likely to come from jet fragmentation, we can expect that the two-particle correlation functions are more characteristic of jet profiles when $p_{T \text { cut }}$ is larger. This method of two-particle correlations is unique because it can determine contributions to particle production from minijets which are intangible under the traditional cluster-finding algorithm 83] due to their small $p_{T} \sim 2 \mathrm{GeV} / c$.

The normalized two-particle correlation functions in the azimuthal angle $\phi$ are defined as

$$
c\left(\phi_{1}, \phi_{2}\right)=\frac{\rho\left(\phi_{1}, \phi_{2}\right)}{\rho\left(\phi_{1}\right) \rho\left(\phi_{2}\right)}-1
$$

where $\rho(\phi)$ is the averaged particle density in $\phi$ and $\rho\left(\phi_{1}, \phi_{2}\right)$ is the two-particle density which is proportional to the probability of joint particle production at $\phi_{1}$ and 


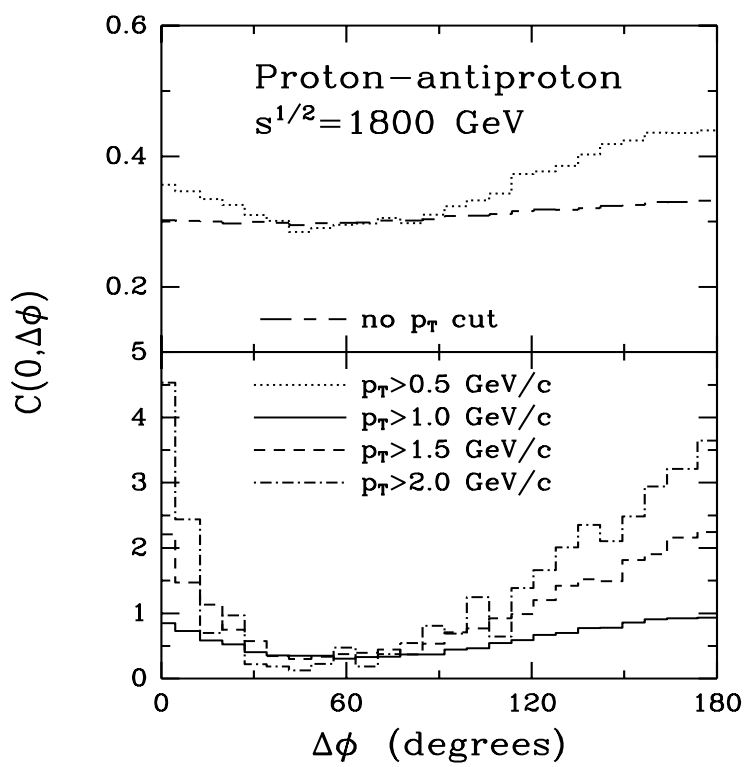

Figure 7: The correlation functions $c(0, \Delta \phi)$ vs $\Delta \phi$ between two charged particles in the full rapidity range in $p \bar{p}$ collisions at $\sqrt{s}=1.8 \mathrm{TeV}$. The dashed line is for all charged particles, dotted line for particles with $p_{T}>0.5 \mathrm{GeV} / c$, dash-dotted line for $p_{T}>1.0 \mathrm{GeV} / c$, and solid line for $p_{T}>1.5 \mathrm{GeV} / c$.

$\phi_{2}$.

Shown in Fig. 7 are our calculated results on two-particle correlation functions in $p \bar{p}$ collisions at $\sqrt{s}=1.8 \mathrm{TeV}$ for selected particles with different $p_{T \text { cut }}$. The calculation includes all charged particles in the full rapidity range. As we have expected, due to minijets, there is strong two-particle correlation at both $\Delta \phi=0$ and $\pi$, forming a valley at $\Delta \phi \sim \pi / 3$. For large $p_{T \text { cut }}$, the correlation functions are very similar to large $p_{T}$ jet profiles as functions of $\phi$ relative to the triggered jet axis 85.]. These features are, however, absent in low-energy hadronic collisions where minijet production is negligible [82]. Since there are still many particles from soft production which can contribute only to the backward correlation due to momentum conservation, the study of energy dependence of the relative heights of the two peaks at $\Delta \phi=0$ and $\pi$ could provide us information about the energy dependence of minijet production. The background at $\Delta \phi \sim \pi / 3$ also depends on the average number of minijets produced 82$]$.

Unlike high $p_{T}$ back-to-back jets which are both kinematically bounded to the central rapidity region, a pair of minijets can be easily produced with a large rapidity gap between them. When we trigger one minijet in a limited rapidity window, the other one which is produced in the same parton scattering often falls outside the fixed rapidity window. Therefore, if we calculate two-particle correlations for particles in a 


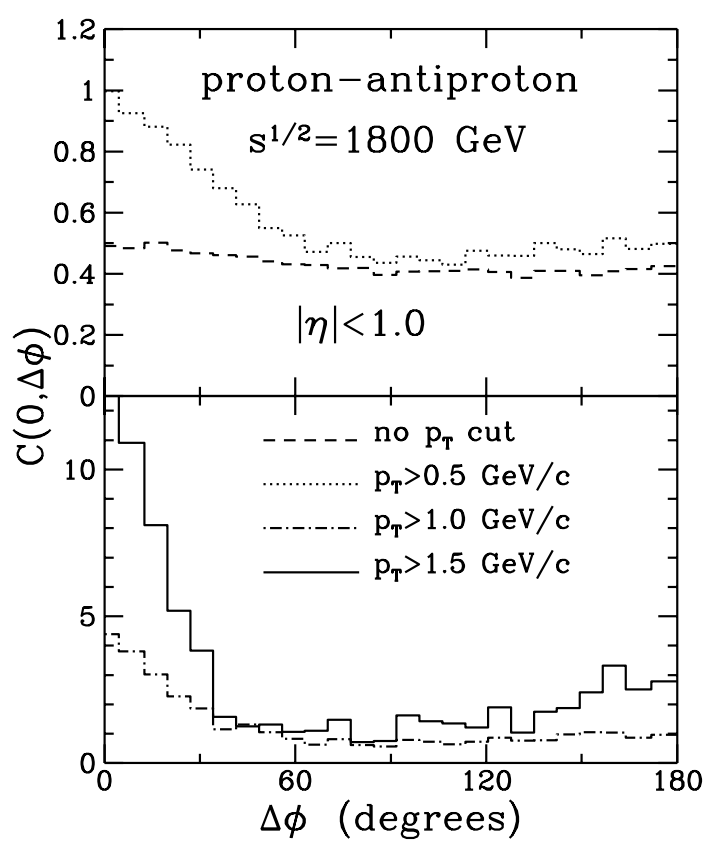

Figure 8: The same as Fig. 7, except for charged particles in a limited rapidity range of $|\eta|<1$.

limited rapidity range, the minijet contribution to the backward correlation $(\Delta \phi=\pi)$ will mostly drop out while the contribution to forward correlation $(\Delta \phi=0)$ still remains. Indeed, as shown in Fig. 8 , the forward correlation at $\Delta \phi=0$ for particles in $|\eta|<1$ is very strong, but the backward correlation at $\Delta \phi=\pi$ is drastically reduced as compared to the correlation pattern in the full rapidity range in Fig. 7. Furthermore, due to strong short range two-particle correlations in rapidity [74], the forward correlation at $\Delta \phi=0$ is also enhanced by restricting particles to $|\eta|<1$. At $\sqrt{s}=1.8 \mathrm{TeV}$, we find that the enhancement of backward correlation at $\Delta \phi=\pi$ due to minijets becomes important only when the rapidity window is $|\eta| \gtrsim 2$.

\section{Parton Production in Nucleus-Nucleus Collisions}

When we extrapolate the model to hadron-nucleus and nucleus-nucleus collisions, nuclear effects due to multiple parton scatterings and the interference have to be considered. Those nuclear effects can be generally divided into the two categories of initial and final state interactions. Initial state interactions and the interference lead to an apparent depletion of the effective parton density inside a nucleus, the so-called nuclear shadowing. They also lead to a modification of the momentum spectra of 
produced partons, the Cronin effect [86], at intermediate energies. However, as I will demonstrate below, the Cronin effect will disappear at large transverse momentum and at high energies, like other high-twist processes. Both initial and final state scatterings can cause a fast parton to lose its energy. Due to color interference, this energy loss is not directly proportional [27, 31, 32] to the parton's energy. Thus for extremely energetic initial partons, this energy loss will be negligible. However, for produced partons which travel in the transverse direction, the energy loss becomes important relative to their finite transverse momentum. This energy loss will essentially modify the parton fragmentation, as well as accelerate parton equilibration.

\subsection{Nuclear Shadowing of Parton Distribution Functions}

Let us start first with the effect of nuclear modification of the parton distributions, or nuclear shadowing due to initial state interactions. What we are mostly concerned about here is the depletion of the effective parton density which will reduce the initial produced parton density and the transverse energy density [19, 87, 88]. This Section is based mainly on the work by Eskola, Qiu and myself in Ref. [100].

"Nuclear shadowing" in the context of the deeply inelastic lepton-nucleus scattering refers to the measured depletion of the nuclear structure function $F_{2}^{A}$ at small

$x_{\mathrm{Bj}}$, as compared to $F_{2}$ of unbound nucleons [89]. The same kind of depletion at small $x$ is expected to happen also in the nuclear gluon distributions. During the recent years there have been many efforts to explain the measured nuclear shadowing of quarks and antiquarks [28, 90, 91, 92, 93, 94], but for gluons the situation is still inconclusive. In these models, shadowing at small $x$ can be attributed to parton fusions before the hard scattering which probes the parton distributions. In terms of parton fusions, shadowing is also predicted to happen in protons. In this case, "shadowing" refers to the saturation of the actual parton distributions caused by fusions of overcrowding gluons at very small $x$. This mechanism proceeds through perturbative QCD-evolution as formulated in [37, 95]. It has been shown by Collins and Kwieciński that the singular gluon distributions actually saturate due to gluon fusions [96]. I will first review QCD-evolution of parton distributions and then demonstrate how parton shadowing arises from the inclusion of a fusion term in the evolution equation.

Parton distribution functions inside a nucleon or nucleus are closely related to the Pomeron substructure and its coupling to quarks and gluons [37, 46]. If one assumes that the ladder diagram inside a Pomeron is ordered in $p_{T}$, then one can derive a set of evolution equations for the parton distribution functions with respect 
to the momentum scale of the hard scattering [42, 97]:

$$
\begin{aligned}
Q^{2} \frac{\partial q_{i}\left(x, Q^{2}\right)}{\partial Q^{2}} & =\frac{\alpha_{s}\left(Q^{2}\right)}{2 \pi} \int_{x}^{1} \frac{d y}{y}\left[P_{q \rightarrow q g}(y) q_{i}\left(\frac{x}{y}, Q^{2}\right)+P_{g \rightarrow q \bar{q}}(y) g\left(\frac{x}{y}, Q^{2}\right)\right] \\
Q^{2} \frac{\partial g\left(x, Q^{2}\right)}{\partial Q^{2}} & =\frac{\alpha_{s}\left(Q^{2}\right)}{2 \pi} \int_{x}^{1} \frac{d y}{y}\left[\sum_{i=1}^{2 N_{f}} P_{q \rightarrow g q}(y) q_{i}\left(\frac{x}{y}, Q^{2}\right)+P_{g \rightarrow g g}(y) g\left(\frac{x}{y}, Q^{2}\right)\right]
\end{aligned}
$$

where the splitting functions, $P_{a \rightarrow b c}(y)$, are the probability distribution functions for the respective radiative processes as illustrated in Fig. 9(a), $x$ is the fractional momentum of the specified partons, and $N_{f}$ is the number of quark flavors. In addition to these splitting processes, one should also take into account the virtual corrections as shown in Fig. 9(b). Their contributions to the evolution equations are 98

$$
\begin{array}{ll}
q_{i}\left(x, Q^{2}\right): & -\frac{\alpha_{s}\left(Q^{2}\right)}{2 \pi} q_{i}\left(x, Q^{2}\right) \int_{0}^{1} d y P_{q \rightarrow q g}(y), \\
g\left(x, Q^{2}\right): & -\frac{\alpha_{s}\left(Q^{2}\right)}{2 \pi} g\left(x, Q^{2}\right) \int_{0}^{1} d y \frac{1}{2}\left[P_{g \rightarrow g g}(y)+N_{f} P_{g \rightarrow q \bar{q}}(y)\right] .
\end{array}
$$

These virtual corrections are important to regularize the soft divergences in the splitting functions and guarantee flavor and momentum conservation. If one wants a probabilistic interpretation of the evolution and especially a numerical simulation, the above virtual corrections will give rise to the Sudakov form factors which are necessary to guarantee unitarity. As one can see, the above virtual corrections can be incorporated into the evolution equations by introducing a $\delta$-function and "+functions" (see [97 for their definitions) in the splitting functions

$$
\begin{aligned}
P_{g \rightarrow q \bar{q}}(y) & =\frac{1}{2}\left[y^{2}+(1-y)^{2}\right] \\
P_{q \rightarrow g q}(y) & =\frac{4}{3} \frac{1+(1-y)^{2}}{y} ; \\
P_{q \rightarrow q g}(y) & =\frac{4}{3}\left[\frac{1+y^{2}}{(1-y)_{+}}+\frac{3}{2} \delta(1-y)\right] ; \\
P_{g \rightarrow g g}(y) & =6\left[\frac{y}{(1-y)_{+}}+\frac{1-y}{y}+y(1-y)+\frac{1}{12}\left(11-\frac{2}{3} N_{f}\right) \delta(1-y)\right] .
\end{aligned}
$$

With the above splitting functions one can check that flavor and momentum are 


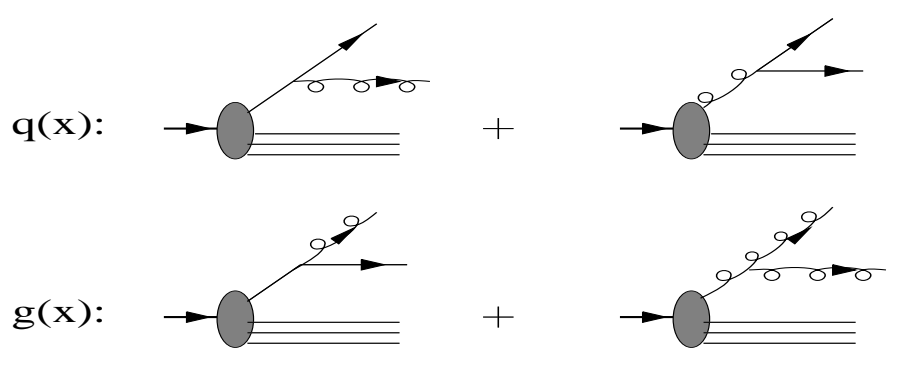

(a)
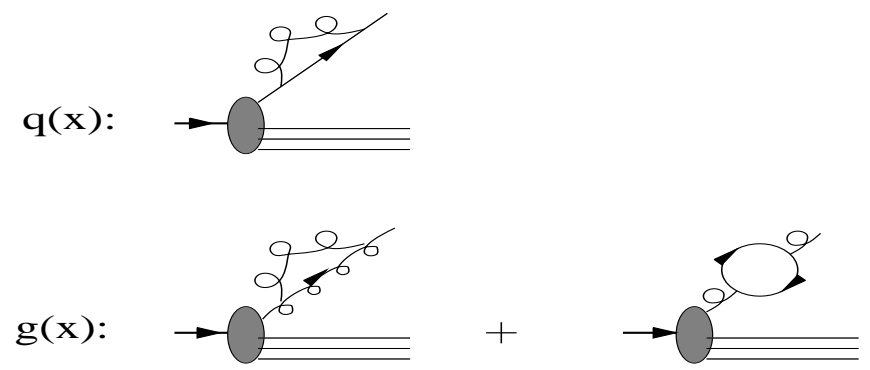

(b)

Figure 9: (a) Radiative and (b) virtual corrections to parton distributions.

conserved, i.e.,

$$
\begin{aligned}
\frac{\partial}{\partial Q^{2}} \int_{0}^{1} d x\left[q_{i}\left(x \cdot Q^{2}\right)-\bar{q}_{i}\left(x, Q^{2}\right)\right] & =0 \\
\frac{\partial}{\partial Q^{2}} \int_{0}^{1} d x x\left[g\left(x, Q^{2}\right)+\sum_{i=1}^{2 N_{f}} q_{i}\left(x, Q^{2}\right)\right] & =0 .
\end{aligned}
$$

In the parton model, a nucleus consists of many valence quarks. Those valence quarks, however, are dressed with clouds of sea quarks and gluons due to vacuum fluctuations (which corresponds to the loops inside a Pomeron ladder). The life time of the virtual partons is of the order of the hadron size, $R_{N} \sim 1 \mathrm{fm}$. If those valence quarks are probed by a hard scattering with resolution $Q \gg 1 / R_{N}$, the coherence of the parton clouds will be broken and numerous partons will be released. The parton density inside the clouds will then depend on the resolution of the scattering $Q$. The larger the $Q$ the denser the parton clouds, as predicted by the QCD evolution equations. These evolution equations are based on perturbative QCD which can only be applied down to some scale $Q_{0}$. Given the initial values of parton distributions at $Q_{0}$, the evolution in $Q>Q_{0}$ can be predicted by pQCD. However, the parton distributions below $Q_{0}$ cannot be obtained from pQCD and so far can only be determined from experiments. 
At small values of $x$, the leading order QCD evolution equations predict that the number of gluons becomes extremely large. It has been known [37, 95] that for sufficiently small values of $x$ and/or of $Q^{2}$, the total transverse area occupied by the gluons will be larger than the transverse area of a hadron, so that the interactions among gluons can no longer be neglected. Such gluon recombination results in a modification of the QCD evolution equations, Eqs. (39) and (40). In the limit of small- $x$ and neglecting quark distributions, the modified QCD evolution equation for the gluon distribution can be cast in the form 37, 95

$$
\partial_{y} \partial_{t} G(y, t)=c G(y, t)-\gamma \exp \left(-t-\mathrm{e}^{t}\right)[G(y, t)]^{2},
$$

where $y=\ln (1 / x), t=\ln \left[\ln \left(Q^{2} / \Lambda_{\mathrm{QCD}}^{2}\right)\right], G(y, t)=x g\left(x, Q^{2}\right)$ and $c=12 /\left(11-2 N_{f} / 3\right)$ with $N_{f}$ the number of quark flavors.

The seond term in Eq. (49) corresponds to the change of the distribution due to gluon recombination. The strength of the gluon recombination is controlled by the factor $\gamma$, originating from two possible sources. One can consider gluon fusion in terms of one Pomeron to two-Pomeron coupling. The two fusing gluon ladders (two Pomerons), which couple 4 gluons to 2 gluons, can arise either from independent constituents of the proton/nucleus or from the same one, as discussed in 95, 96, 99]. We will refer to the former case as "independent" and to the latter as "nonindependent" fusion. Since recombinations from both sources happen simultaneously, we divide the parameter $\gamma$ into two parts:

$$
\gamma=\gamma_{\mathrm{I}}+\gamma_{\mathrm{II}}
$$

where $\gamma_{\mathrm{I}}$ corresponds to the independent recombination and $\gamma_{\mathrm{II}}$ to the non-independent one.

To understand the form of the fusion term in Eq. (49), one can consider $x g\left(x, Q^{2}\right)$ as the gluon number $n_{g}$ per unit rapidity $(d y=d x / x)$. If we note that the gluon-gluon cross section $\hat{\sigma} \sim \alpha_{s} / \Lambda_{\mathrm{QCD}}^{2}$, the independent gluon fusion probability inside a nucleon with transverse area $\pi R_{N}^{2}$ is then,

$$
W=\frac{n_{g}^{2} \hat{\sigma}}{\pi R_{N}^{2}} \sim \frac{\alpha_{s}}{R_{N}^{2} \Lambda_{\mathrm{QCD}}^{2}}\left[x g\left(x, Q^{2}\right)\right]^{2} .
$$

This corresponds to the gluon fusion term in Eq. (49) (note that $e^{-t} \sim \alpha_{s}$ ). One can calculate this fusion term in a QCD recombination model [95]. In a proton, the 
strength of the independent fusion then takes the form

$$
\gamma_{\mathrm{I}}=\frac{2}{3} \frac{1}{\pi R_{N}^{2}} \cdot \frac{\pi^{3} c^{2}}{2 \Lambda_{\mathrm{QCD}}^{2}},
$$

where $R_{N} \sim 1 \mathrm{fm}$ is the radius of a proton.

The non-independent fusion happens inside the same valence quark which is assumed to have a transverse size $\sim 1 / Q_{i}$. The magnitude of the non-independent fusion of the gluon ladders can be estimated as 95

$$
\gamma_{\mathrm{II}} \approx \frac{16}{81} \frac{1}{\pi\left(2 / Q_{i}\right)^{2}} \cdot \frac{\pi^{3} c^{2}}{2 \Lambda_{\mathrm{QCD}}^{2}},
$$

where a simplification is made by fixing the initial $x$ of the valence quark to $x_{i} \sim 1$. We also approximate the scale of the initial valence quark by $Q_{i} \sim 2 \mathrm{GeV}$.

Let us then consider a large loosely bound nucleus. Naturally, both types of fusions are still there but only for the independent one an $A^{1 / 3}$-scaling arises. In this case

$$
\gamma_{\mathrm{I}}^{A}=\frac{9}{8} \frac{A}{\pi R_{A}^{2}} \cdot \frac{\pi^{3} c^{2}}{2 \Lambda_{\mathrm{QCD}}^{2}},
$$

where the nucleus is taken to be a sphere with a sharp surface at $R_{A}=1.12 A^{1 / 3} \mathrm{fm}$. The strength of the non-independent fusion remains the same as in the case of a free proton: $\gamma_{\mathrm{II}}^{A}=\gamma_{\mathrm{II}}$.

It is interesting to notice how the relative contributions of the two types of recombination will change when going from a proton to a nucleus of $A \sim 200: \gamma_{\mathrm{II}} / \gamma_{\mathrm{I}} \approx$ 7.6 and $\gamma_{\mathrm{II}}^{A} / \gamma_{\mathrm{I}}^{A} \approx 1.0$. Thus the non-independent fusion is clearly dominant in a free proton while in a large nucleus the contributions from both types are of the same order. As a result, parton recombination is strongly enhanced in a heavy nucleus.

In order to solve Eq. (49) exactly by integration, one would need the initial distribution either at fixed $y_{0}$ or $t_{0}$ and the derivatives along a boundary line $\left(y, t_{0}\right)$ or $\left(y_{0}, t\right)$, respectively. However, since the expression for the non-linear term in Eq. (49) is not valid for the regions where $x$ is large, or where both $x$ and $Q$ are very small, the natural boundary condition at $x=1$ ( or $y_{0}=0$ ) is not suitable here. In addition, since we do not have sufficient information on other boundary lines, we cannot solve Eq. (49) by direct integration. Instead, with the semiclassical approximation [37, we use the method of characteristics [96, 100], so that we can avoid the region of both small $x$ and small $Q^{2}$.

The semiclassical approximation corresponds to neglecting the second order 
derivative term, $\partial_{y} \partial_{t} \ln (G)$, which leaves us with the evolution equation as

$$
\partial_{y} z(y, t) \partial_{t} z(y, t)=c-\gamma \exp \left[-t-\mathrm{e}^{t}+z(y, t)\right],
$$

where $z(y, t)=\ln [G(y, t)]$. The reason why this approximation is called semiclassical is that it corresponds to using a saddle-point approximation to the integration in the integral form of the evolution equation. The above equation can then be cast and solved in the form of a set of characteristic equations as shown in detail in 96. What is needed here is the gluon distribution at the boundary $y_{0}\left[=-\ln \left(x_{0}\right)\right]$ at all $t$, which can be obtained by evolving the distribution in the region $x>x_{0}$ according to the original GLAP evolution equations, Eqs. (39) and (40).

For a proton, we assume that the recombination becomes effective at $x \sim x_{0} \sim$ 0.01 , which is consistent with [96, 99]. We can use the results from a global fitting to the parton distributions, like CTEQ [101], to constrain $x_{0}$. In fact, we will see that with $x_{0}=0.01$ the shadowed gluons deviate considerably from the CTEQ gluons only after $x<0.001$, so our choice for $x_{0}$ seems to be reasonable, and we do not expect the results to be very sensitive to small changes of $x_{0}$.

As explained above, the gluon recombination is strongly enhanced in heavy nuclei and it starts at somewhat larger values of $x$ than in protons. The corresponding boundary line $x_{0}^{A}$ for a nucleus is approximately determined by the relative magnitude of the evolution terms in Eq. (49): for $G_{A}\left(x_{0}^{A}\right) \sim G\left(x_{0}\right) \gamma_{A} / \gamma$, the relative contribution from the gluon fusion in a nucleus is about the same as in a nucleon. This gives $x_{0}^{A} \sim$ $0.05-0.1$. This range of $x_{0}^{A}$ is also supported by other studies 102.

Let the total gluonic fractional momentum in the non-shadowed parton distributions be $f_{0}=\int_{0}^{1} d x x g_{\mathrm{CTEQ}}\left(x, Q_{0}^{2}\right)$. In the case of a proton, shadowing changes the gluonic momentum typically by less than a per cent, which we can clearly neglect as a small overall change.

Perturbative shadowing at small $x$ reduces the gluonic momentum more in a nucleus than in a proton. If the momentum fraction of gluons is conserved, there must be a corresponding enhancement in the large $x$ region. However, there can also be momentum transfers from quarks and antiquarks to the gluons. Here we consider nuclei with $A \sim 200$, for which we expect an overall increase in the gluonic momentum fraction, $\epsilon_{A}$, to be only about $4 \%$ [91, 92, 102] as constrained by the experimental data on quark (anti-quark) shadowing in deep inelastic scatterings. These two sources of momentum flow will result in anti-shadowing (enhancement of parton densities) at large $x>x_{0}^{A}$. To account for the anti-shadowing we assume $g\left(x, Q_{0}^{2}\right)=a_{A} g_{\mathrm{CTEQ}}\left(x, Q_{0}^{2}\right)$ for $x>x_{0}^{A}$ with $g_{\mathrm{CTEQ}}\left(x, Q_{0}^{2}\right)$ the non-shadowed gluon 
distribution and $a_{A}>1$ to be determined by the momentum sum rule,

$$
\begin{aligned}
\left.\int_{0}^{x_{0}^{A}} d x x g\left(x, Q_{0}^{2}\right)\right|_{C} & +a_{A} \int_{x_{0}^{A}}^{1} d x x g_{\mathrm{CTEQ}}\left(x, Q_{0}^{2}\right) \\
& =f_{0}\left(1+\epsilon_{A}\right) .
\end{aligned}
$$

The right-hand-side of the equation is the gluonic momentum fraction of the nonshadowed parton distributions plus the momentum transfer $\epsilon_{A}$ from quarks and antiquarks during the recombination. One can solve the above equation iteratively for $a_{A}$ with the boundary condition $C: g_{A}\left(x_{0}^{A}, Q_{0}^{2}\right)=a_{A} g_{\mathrm{CTEQ}}\left(x_{0}^{A}, Q_{0}^{2}\right)$. Typically, $a_{A} \sim 1.1$ for $A \sim 200$.

In Fig. 10(a), nucleon and effective nuclear gluon distributions for a nucleus of $A=200$ are compared with the input CTEQ gluon distribution at $Q_{0}=2 \mathrm{GeV}$. Notice the $\sim 20 \%$ uncertainty in the nuclear case resulting from varying $x_{0}^{A}$ from 0.05 to 0.1 . To demonstrate the formation of strong perturbative nuclear shadowing, corresponding to the relative depletion of gluon distributions in a nucleus, we plot the ratio $G_{A}\left(x, Q_{0}^{2}\right) / G\left(x, Q_{0}^{2}\right)$ in Fig. 10(b). Notice also that as $x$ decreases, the gluon distribution in a proton increases much faster, or approaches saturation at a much smaller $x$ than that in a nucleus. Therefore, as shown in Fig. 10(b) the ratio saturates only when the gluons in a proton do so. Thus, saturation of the perturbative nuclear shadowing reflects actually the behavior of the gluons in a proton. We see that, due to the enhanced gluon recombination in a heavy nucleus, a 50\% nuclear shadowing in small- $x$ region is generated perturbatively through the modified QCD evolution, accompanied by a $\sim 10 \%$ antishadowing from momentum conservation.

We have not included the non-perturbative, e.g., Glauber-derived [28] contribution to the shadowing. Inclusion of such a contribution is equivalent to changing the initial values of gluon distribution at scale $Q_{0}$ and will slightly reduce the perturbative contribution to the shadowing through QCD evolution. We also have not considered the shadowing of quark distributions in this study of perturbative shadowing. However, experimental data [89] on quark shadowing are roughly consistent with the perturbative gluon shadowing we have just estimated. The shadowing, at least for quarks, has been shown to depend weakly on the scale $Q$ [102, also consistent with experimental data.

For a practical implementation of the nuclear shadowing in HIJING, we consider that quarks and gluons are shadowed by the same amount inside a nucleus and use the following parametrized form:

$$
R_{A}(x) \equiv \frac{f_{a / A}(x)}{A f_{a / N}(x)}
$$




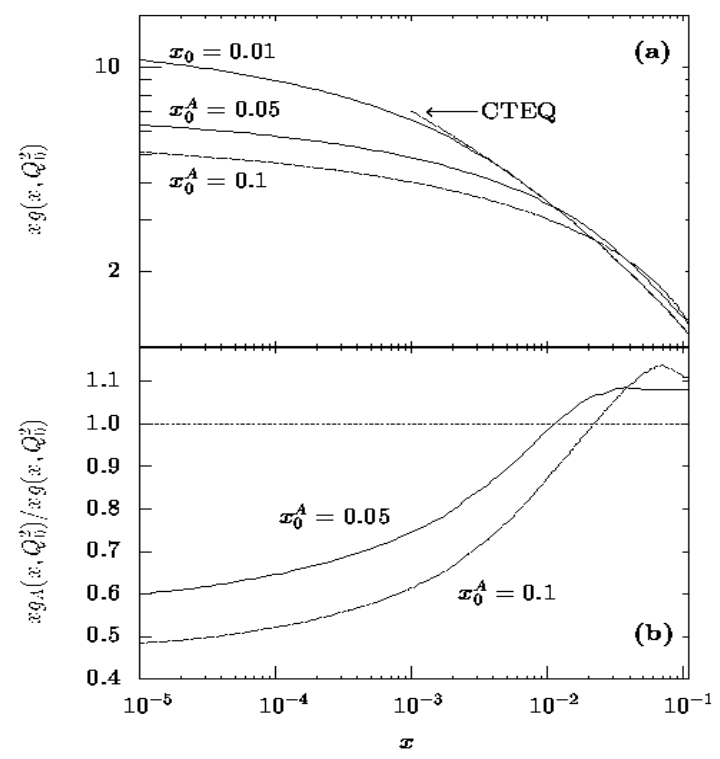

Figure 10: (a) The gluon distributions $x g\left(x, Q_{0}^{2}\right)$ at $Q_{0}=2 \mathrm{GeV}$ vs. $x$. The result for proton is labeled by $x_{0}$, and the results for $A \sim 200$ by $x_{0}^{A}$, respectively. The CTEQ gluon distribution [101] is labeled by "CTEQ". (b) The ratio $x g_{A}\left(x, Q_{0}^{2}\right) / x g\left(x, Q_{0}^{2}\right)$ of the shadowed gluon distributions vs. $x$, demonstrating a strong perturbative nuclear shadowing in heavy nuclei.

$$
\begin{aligned}
= & 1+1.19 \ln ^{1 / 6} A\left[x^{3}-1.5\left(x_{0}+x_{L}\right) x^{2}+3 x_{0} x_{L} x\right] \\
& -\left[\alpha_{A}-\frac{1.08\left(A^{1 / 3}-1\right)}{\ln (A+1)} \sqrt{x}\right] e^{-x^{2} / x_{0}^{2}}, \\
\alpha_{A}= & 0.1\left(A^{1 / 3}-1\right),
\end{aligned}
$$

where $x_{0}=0.1$ and $x_{L}=0.7$. The term proportional to $\alpha_{A}$ in Eq. (57) determines the shadowing for $x<x_{0}$ with the most important nuclear dependence, while the rest gives the overall nuclear effect on the structure function in $x>x_{0}$ with a very weak $A$ dependence. As shown in Fig. 11, this parametrization reproduces the measured overall nuclear effect on the quark structure function in the small and medium $x$ regions. However, I should emphasize that this parametrization does not satisfy the momentum sum rule and does not include the weak scale dependence which was found by Eskola in a detailed study in Ref. 102.

Eq. (57) represents only the average nuclear dependence of the structure function. However, in $p A$ or $A A$ collisions, we have to calculate the effective jet cross sections at the nucleon-nucleon level for each impact parameter. Physically, it is natural to expect that the nuclear effects on the structure functions could depend on the local nuclear thickness at each impact parameter [87]. Eq. (58) is consistent with the 


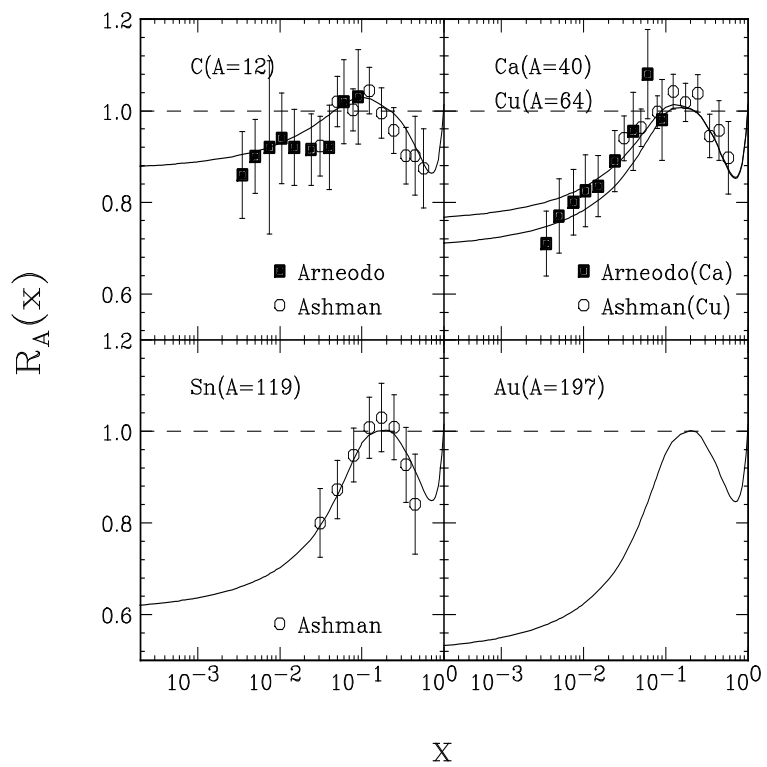

Figure 11: The ratio of quark structure functions $R_{A}(x) \equiv F_{2}^{A}(x) / A F_{2}^{N}(x)$ as a function of $x$ in small and medium $x$ regions for different nuclear mass numbers $A$. The data are from Ref. [89] and curves are the parametrization in Eqs. (57) and (58).

assumption that the shadowing parameter $\alpha_{A}(r)$ is proportional to the longitudinal thickness of the nucleus at impact parameter $r$. We therefore parameterize the impact parameter dependence of $\alpha_{A}$ in Eq. (57) as

$$
\alpha_{A}(r)=0.1\left(A^{1 / 3}-1\right) \frac{4}{3} \sqrt{1-r^{2} / R_{A}^{2}},
$$

where $r$ is the transverse distance of the interacting nucleon from its nucleus center and $R_{A}$ is the radius of the nucleus. For a sharp-sphere nucleus with thickness function $T_{A}(r)=\frac{3 A}{2 \pi R_{A}^{2}} \sqrt{1-r^{2} / R_{A}^{2}}$, the averaged $\alpha_{A}(r)$ is $\int_{0}^{R_{A}^{2}} \pi d r^{2} T_{A}(r) \alpha_{A}(r) / A=\alpha_{A}$, with $\alpha_{A}$ from Eq. (58). Because the rest of Eq. (57) has a weaker $A$ dependence, we only consider the impact parameter dependence of $\alpha_{A}$.

To simplify the calculation during the Monte Carlo simulations, we can decompose $R_{A}(x, r)$ into two parts,

$$
R_{A}(x, r) \equiv R_{A}^{0}(x)-\alpha_{A}(r) R_{A}^{s}(x),
$$

where $\alpha_{A}(r) R_{A}^{s}(x)$ is the term proportional to $\alpha_{A}(r)$ in Eq. (57) with $\alpha_{A}(r)$ given in Eq. (59) and $R_{A}^{0}(x)$ is the rest of $R_{A}(x, r)$. Both $R_{A}^{0}(x)$ and $R_{A}^{s}(x)$ are now independent of $r$. The effective jet production cross section of a binary nucleon- 
nucleon interaction in $A+B$ nuclear collisions can therefore be decomposed as 87

$$
\sigma_{\text {jet }}^{e f f}\left(r_{A}, r_{B}\right)=\sigma_{\text {jet }}^{0}-\alpha_{A}\left(r_{A}\right) \sigma_{\text {jet }}^{A}-\alpha_{B}\left(r_{B}\right) \sigma_{\text {jet }}^{B}+\alpha_{A}\left(r_{A}\right) \alpha_{B}\left(r_{B}\right) \sigma_{\text {jet }}^{A B}
$$

where $\sigma_{\text {jet }}^{0}, \sigma_{\text {jet }}^{A}, \sigma_{\text {jet }}^{B}$ and $\sigma_{\text {jet }}^{A B}$ can be calculated through Eq. (19) by multiplying $f_{a}\left(x_{1}, p_{T}^{2}\right) f_{b}\left(x_{2}, p_{T}^{2}\right)$ in the integrand with $R_{A}^{0}\left(x_{1}\right) R_{B}^{0}\left(x_{2}\right), R_{A}^{s}\left(x_{1}\right) R_{B}^{0}\left(x_{2}\right)$, $R_{A}^{0}\left(x_{1}\right) R_{B}^{s}\left(x_{2}\right)$ and $R_{A}^{s}\left(x_{1}\right) R_{B}^{s}\left(x_{2}\right)$, respectively.

In central $A u+A u$ collisions at $\sqrt{s}=200 \mathrm{AGeV}$, the average parton fractional momentum is $x=2 p_{T} / \sqrt{s} \simeq 0.02$ for mini-jets with $p_{T} \geq p_{0}=2 \mathrm{GeV}$. The impactparameter dependent parton shadowing reduces the averaged inclusive mini-jet cross section by $50 \%$ from its value in $p p$. This estimate will be somewhat modified if one includes the scale dependence of the shadowing effect. However, at RHIC energies we can see from Fig. 11 that mini-jet production with $x \simeq 0.02$ is still not in the deepshadowed region of $x$. For sufficiently high energies, most of the mini-jets come from $x \lesssim 0.01$ region so that the effective mini-jet cross section may be reduced by a factor 3 in central $A u+A u$ collisions. Note that this large reduction of mini-jet multiplicity by gluon shadowing may increase the limit for independent multi-jet production up to $\sqrt{s}=50 \mathrm{ATeV}$ for $A u+A u$ collisions.

\subsection{Disappearance of Cronin Effect at High Energies}

In hadron-nucleus and nucleus-nucleus collisions, multiple scatterings happen both at hadronic and partonic levels. Due to the interference between multiple scattering amplitudes, a hadron can only "see" the surface of a nucleus, leading to a total cross section $\sim R_{A}^{2}$. Parton fusions which cause nuclear shadowing of the parton distribution functions can also be viewed as multiple parton scattering in the laboratory frame. The parton depletion arises from the destructive interference among different scattering amplitudes [28]. These multiple scatterings and their interference will also affect the momentum spectra of the produced partons. In general, as observed first by Cronin et al. [86], particle production at large $p_{T}$ will be enhanced. However, recent experiments 103 show that the enhancement decreases both with $p_{T}$ and energy $\sqrt{s}$.

I would like to emphasize that the initial multiple scatterings discussed here are very different from the late final parton rescatterings which are responsible for parton thermalization. The initial partons in general have very small transverse momenta but large center-of-mass (c.m.) energies. As demonstrated in Ref. [27, multiple scatterings among these initial partons cannot be treated as semiclassical cascades which might be valid for final parton rescatterings. The eikonal limit is more relevant for the initial partons scatterings which can be treated by a Glauber multiple scattering analysis. 
Following the same procedure that leads to the hadron-hadron scattering cross section due to exchange of multiple Pomerons, Eq. (14), one can also obtain the inelastic cross section for $p A$ collisions,

$$
\sigma_{p A}(s)=\int d^{2} b\left[1-e^{-\sigma_{N N}(s) T_{A}(b, s)}\right],
$$

where $\sigma_{N N}$ is the total nucleon-nucleon cross section and

$$
T_{A}(b)=\int_{-\infty}^{\infty} d z \rho(b, z)
$$

is the thickness function of the nucleus at impact parameter $b$. The nuclear density, $\rho(b, z)$, is normalized to $\int d^{2} b T_{A}(b)=A$.

Let us assume that the total nucleon-nucleon cross section can be described by parton scatterings with given cross sections. Define the differential cross section for a parton-nucleon scattering, $i+N \rightarrow j+X$, as $h^{i j}\left(p_{i}, p_{j}\right)$, with the total cross section

$$
\sigma_{i}\left(p_{i}\right)=\frac{1}{2} \sum_{j} \frac{d^{3} p_{j}}{E_{j}} h^{i j}\left(p_{i}, p_{j}\right) .
$$

Let us also define

$$
T_{A}^{ \pm}(b, z)= \pm \int_{z}^{ \pm \infty} d z^{\prime} \rho\left(b, z^{\prime}\right)
$$

Note that

$$
T_{A}(b)=T_{A}^{-}(b, z)+T_{A}^{+}(b, z) .
$$

Now according to a Glauber multiple scattering interpretation of the $p A$ cross section Eq. (62), the probabilities for parton $i$ not to interact with the nucleons up to $z$ and for $j$ not to interact after $z$, are respectively,

$$
e^{-\sigma_{i}\left(p_{i}\right) T_{A}^{-}(b, z)}, \quad e^{-\sigma_{j}\left(p_{j}\right) T_{A}^{+}(b, z)}
$$

Then, the probability for one parton-nucleon scattering, $i+A \rightarrow j+X$, is

$$
\begin{aligned}
\frac{d H_{(1)}^{i j}}{d^{2} b} & =\int_{-\infty}^{\infty} d z e^{-\sigma_{i} T_{A}^{-}(b, z)} \rho(b, z) h^{i j} e^{-\sigma_{j} T_{A}^{+}(b, z)} \\
& =\frac{h^{i j}}{\sigma_{j}-\sigma_{i}}\left[e^{-\sigma_{i} T_{A}(b)}-e^{-\sigma_{j} T_{A}(b)}\right] \\
& =h^{i j} T_{A}(b)\left[1-\frac{\sigma_{i}+\sigma_{j}}{2} T_{A}(b)\right]+\cdots
\end{aligned}
$$


where the definition of the thickness functions $T_{A}(b)$ and Eq. (66) have been used, and an expansion in terms of $\sigma_{i, j} T_{A}(b)$ has been made in the last step.

We can use the above result to calculate the probability for a parton $k$ to have only one scattering after $z$, by replacing $T_{A}(b)$ with $T_{A}^{+}(b, z)$. Then the contribution to $i+A \rightarrow j+X$ from two parton-nucleon scatterings is

$$
\begin{aligned}
\frac{d H_{(2)}^{i j}}{d^{2} b} & =\sum_{k} \int_{-\infty}^{\infty} d z e^{-\sigma_{i} T_{A}^{-}(b, z)} \rho(b, z) \frac{h^{i k} h^{k j}}{\sigma_{j}-\sigma_{k}}\left[e^{-\sigma_{k} T_{A}^{+}(b, z)}-e^{-\sigma_{j} T_{A}^{+}(b, z)}\right] \frac{d^{3} p_{k}}{E_{k}} \\
& =\sum_{k} \frac{d^{3} p_{k}}{E_{k}} \frac{h^{i k} h^{k j}}{\sigma_{j}-\sigma_{k}}\left[\frac{e^{\left.-\sigma_{i} T_{A}^{(} b\right)}-e^{-\sigma_{k} T_{A}(b)}}{\sigma_{k}-\sigma_{i}}-\frac{e^{-\sigma_{i} T_{A}(b)}-e^{-\sigma_{j} T_{A}(b)}}{\sigma_{j}-\sigma_{i}}\right] \\
& =\frac{1}{2} T_{A}^{2}(b) \sum_{k} \frac{d^{3} p_{k}}{E_{k}} h^{i k} h^{k j}+\cdots .
\end{aligned}
$$

Therefore, up to second order in $\sigma T_{A}(b)$, we have the cross section for $i+A \rightarrow$ $j+X$

$$
\frac{d H^{i j}}{d^{2} b} \approx h^{i j} T_{A}(b)+\frac{1}{2} T_{A}^{2}(b)\left[\sum_{k} \frac{d^{3} p_{k}}{E_{k}} h^{i k} h^{k j}-\left(\sigma_{i}+\sigma_{j}\right) h^{i j}\right] .
$$

In the second term in the above equation, we can already see that the interference between double and single scattering comes into play. If we assume the parton distribution inside the projectile nucleon to be $f_{i / N}\left(p_{i}\right)$, then the differential cross section for $N+N \rightarrow j+X$ is given by

$$
\sigma_{N N}^{j} \equiv \sum_{i} \int \frac{d^{3} p_{i}}{E_{i}} f_{i / N}\left(p_{i}\right) h^{i j}
$$

Assuming a hard sphere for the nuclear density $\rho(r)$ with a radius $R_{A}=R_{0} A^{1 / 3}$, we then obtain the ratio between the differential cross sections of $N+A \rightarrow j+X$ and $N+N \rightarrow j+X$

$$
R_{A} \equiv \frac{\sigma_{N A}^{j}}{A \sigma_{N N}^{j}}=1+\frac{9 A^{1 / 3}}{16 \pi R_{0}^{2}} \frac{1}{\sigma_{N N}^{j}} \sum_{i} \int \frac{d^{3} p_{i}}{E_{i}} f_{i / N}\left(p_{i}\right)\left[\sum_{k} \frac{d^{3} p_{k}}{E_{k}} h^{i k} h^{k j}-\left(\sigma_{i}+\sigma_{j}\right) h^{i j}\right] .
$$

Compared to the additive model of hard scatterings, parton production in $N A$ collisions is enhanced due to multiple scattering. The enhancement is proportional to $A^{1 / 3}$, which is the average number of nucleons inside a nucleus along the beam direction. This formula was used successfully [104 to explain the enhancement of large $p_{T}$ particle production in $p A$ collisions.

To demonstrate how this enhancement depends on the transverse momentum 
and colliding energy, let us assume a simple power-law form for the parton-nucleon differential cross section,

$$
h^{i j} \equiv d \sigma / d y d p_{T}^{2}=C / p_{T}^{n}, \quad\left(|y|<\Delta Y / 2, p_{T}>p_{0}\right)
$$

Assuming that all partons are identical, the total cross section is then,

$$
\sigma=\frac{1}{2} \int d y d^{2} p_{T} \frac{C}{p_{T}^{n}}=\frac{\pi \Delta Y C}{(n-2) p_{0}^{n-2}} .
$$

For large $p_{T} \gg p_{0}$, one can evaluate the integral,

$$
\begin{aligned}
\int \frac{d^{3} p_{k}}{E_{k}} h^{i k} h^{k j} & =\int d y d^{2} q_{T} \frac{C}{q_{T}^{n}} \frac{C}{\left(\mathbf{p}_{T}-\mathbf{q}_{T}\right)^{n}} \\
& =\frac{C}{p_{T}^{n}} \sigma\left[2+\frac{n(n-2)}{2}\left(p_{0} / p_{T}\right)^{2}+\mathcal{O}\left(\left(p_{0} / p_{T}\right)^{4}\right)\right]
\end{aligned}
$$

Substituting the above cross sections into Eq. (72), we have,

$$
R_{A}=1+\frac{9 A^{1 / 3}}{16 \pi R_{0}^{2}} \sigma \frac{n(n-2)}{2}\left(p_{0} / p_{T}\right)^{2}+\mathcal{O}\left(\left(p_{0} / p_{T}\right)^{4}\right)
$$

There are a few interesting features in the above estimate. The nuclear enhancement of jet cross sections decreases with $p_{T}$, a general feature of high-twist processes. It also depends on the power $n$ of the differential parton cross section. This feature is a direct consequence of the interference effect, i.e., the cancellation between terms in the single and double scattering amplitudes. From both pQCD calculations and experimental data, we know that the power $n$ decreases with the colliding energy. Thus, the nuclear enhancement of large $p_{T}$ parton production will decrease, and eventually it will disappear at high energies. This trend has already been observed in experiments [103] in the energy range $\sqrt{s}=20-40 \mathrm{GeV}$. A simple way to understand this energy dependence is the following. At low energies, the differential cross section for a single hard scattering decreases very fast with $p_{T}$ (large power). It is then easier for the incident parton to acquire a large $p_{T}$ through two successive scatterings, each with small transverse momentum transfer, than through a single large $p_{T}$ scattering. This is why multiple scatterings in $p A$ collisions cause the enhancement of large $p_{T}$ parton production. As energy increases, the differential cross section for a single scattering decreases less rapidly with $p_{T}$ as compared to low energies. It is no longer more economical to produce a large $p_{T}$ parton through double scatterings than through a single scattering. At extremely high energies, the Cronin 
effect will disappear. Therefore, we will not consider multiple initial scatterings in the following since we only consider parton production in heavy-ion collisions at future collider energies (RHIC and LHC).

\subsection{Monte Carlo Simulations}

Since we have argued that multiple initial parton scatterings will become unimportant at high energies, we can assume a binary approximation for hard scatterings. In that case, multiple hard processes involve only independent pairs of partons. Only very rarely does a given parton suffer two high $p_{T}$ scatterings in one event. As the energy increases the number of partons that can participate in moderate $p_{T}>p_{0}$ processes increases rapidly and the nuclear shadowing phenomenon will also become important at small $x$. However, for the bulk of parton and transverse energy production, the basic independent binary nature of the multiple mini-jet production rate is expected to remain a good approximation as long as Eq. (26) holds.

For soft interactions, a nucleus-nucleus collision is also decomposed into binary collisions involving in general excited or wounded nucleons. Wounded nucleons are assumed to be $q-q q$ string-like configurations that decay on a slow time scale compared to the overlapping time of the nuclei. In the FRITIOF [13] scheme wounded nucleon interactions follow the same excitation law as the original hadrons. In the DPM [14] scheme subsequent collisions essentially differ from the first since they are assumed to involve sea partons instead of valence ones. In HIJING [19] we adopted a hybrid scheme, iterating string-string collisions as in FRITIOF but utilizing DPM-like distributions as in Eqs. (36) and (37). Another difference in the way soft interactions are treated in HIJING is that string-string interactions are also allowed to de-excite as well as to excite the strings further within the kinematic limits. In contrast, in the FRITIOF model multiple interactions are assumed to lead only to excitations of strings with greater mass. Many variations of the algorithm for multiple soft interaction are of course possible as emphasized before. The one implemented in HIJING is simply a minimal model which reproduces essential features of moderate energy $p A$ and $A A$ data.

The number of binary collisions at a given nuclear impact parameter is determined by Glauber geometry. We employ three-parameter Woods-Saxon nuclear densities determined by electron scattering data 105 to compute that geometry as done in ATTILA [15].

For each binary collision, we use the eikonal formalism as given in the previous section to determine the collision probability, elastic or inelastic, and the number of jets it produces. After a hard scattering, the energy of the scattered partons is subtracted from the nucleon and only the remaining energy is used to process the 
soft string excitation. The excited string system minus the scattered hard partons suffers further collisions according to the geometric probabilities.

For each hard scattering, one then has to take into account the corrections due to initial and final state radiations. In an axial gauge and in the leading logarithmic approximation, the interference terms of the radiation drop out. The amplitude for successive radiations has then a simple ladder structure and the probability for multiple emissions becomes the product of each emission [97]. The virtualities of the radiating partons are ordered along the tree, decreasing until a final value $\mu_{0}^{2}$ is reached below which pQCD is no longer valid. This provides the framework for a Monte Carlo simulation of parton showering and its space-time interpretation [106, 107.

At a given vertex of the branching tree, the probability for the off-shell parton $a$ of virtuality $q^{2}<q_{\max }^{2}$ to branch into partons $b$ and $c$ with fractions $z$ and $1-z$ of the light-cone momentum is given by 106, 107

$$
d \mathcal{P}_{a \rightarrow b c}\left(q^{2}, z\right)=\frac{d q^{2}}{q^{2}} d z P_{a \rightarrow b c}(z) \frac{\alpha_{s}\left[z(1-z) q^{2}\right]}{2 \pi} \frac{\mathcal{S}_{a}\left(q_{\max }^{2}\right)}{\mathcal{S}_{a}\left(q^{2}\right)},
$$

where $P_{a \rightarrow b c}(z)$ is the Altarelli-Parisi splitting function 42 for the process $a \rightarrow b c$. By requiring the relative transverse momentum $q_{T}$ of $b$ and $c$ to be real,

$$
q_{T}^{2}=z(1-z)\left(q^{2}-\frac{q_{b}^{2}}{z}-\frac{q_{c}^{2}}{1-z}\right) \geq 0,
$$

and a minimum virtuality $q_{b}^{2}, q_{c}^{2} \geq \mu_{0}^{2}$, the kinematically allowed region of phase space is then,

$$
\begin{aligned}
& 4 \mu_{0}^{2}<q^{2}<q_{\max }^{2} ; \\
& \epsilon(q)<z \quad<1-\epsilon(q), \quad \epsilon(q)=\frac{1}{2}\left(1-\sqrt{1-4 \mu_{0}^{2} / q^{2}}\right) .
\end{aligned}
$$

Note that the '+function' and $\delta$-function due to virtual corrections in the splitting functions in Eqs. (43)-(46) are not in effect in the allowed phase space. Their role has been replaced by the Sudakov form factor $\mathcal{S}_{a}\left(q^{2}\right)$ which is defined as [106, 107]

$$
\mathcal{S}_{a}\left(q^{2}\right)=\exp \left\{-\int_{4 \mu_{0}^{2}}^{q^{2}} \frac{d k^{2}}{k^{2}} \int_{\epsilon(k)}^{1-\epsilon(k)} d z \sum_{b, c} P_{a \rightarrow b c}(z) \frac{\alpha_{s}\left[z(1-z) k^{2}\right]}{2 \pi}\right\}
$$

so that $\Pi_{a}\left(q_{\max }^{2}, q^{2}\right)=\mathcal{S}_{a}\left(q_{\max }^{2}\right) / \mathcal{S}_{a}\left(q^{2}\right)$ is the probability for parton $a$ not to have any branching between $q_{\max }^{2}$ and $q^{2}$. Therefore, the Sudakov form factor in the Monte Carlo simulation is essential to include virtual corrections and ensure unitarity. Since 
the probability of parton emission between $q^{2}$ and $q^{2}-d q^{2}$ is

$$
\frac{d q^{2}}{q^{2}} \int_{\epsilon(k)}^{1-\epsilon(k)} d z \sum_{b, c} P_{a \rightarrow b c}(z) \frac{\alpha_{s}\left[z(1-z) q^{2}\right]}{2 \pi},
$$

the probability of no parton emission, by unitarity, will be

$$
1-\frac{d q^{2}}{q^{2}} \int_{\epsilon(k)}^{1-\epsilon(k)} d z \sum_{b, c} P_{a \rightarrow b c}(z) \frac{\alpha_{s}\left[z(1-z) q^{2}\right]}{2 \pi}
$$

between $q^{2}$ and $q^{2}-d q^{2}$. The probability of no parton emission between $q_{\max }^{2}$ and $q^{2}-d q^{2}$ then will be the product of the two probabilities,

$$
\begin{aligned}
\Pi_{a}\left(q_{\max }^{2}, q^{2}-d q^{2}\right) & =\Pi_{a}\left(q_{\max }^{2}, q^{2}\right)-d \Pi_{a}\left(q_{\max }^{2}, q^{2}\right) \\
& =\Pi_{a}\left(q_{\max }^{2}, q^{2}\right)\left\{1-\frac{d q^{2}}{q^{2}} \int_{\epsilon(k)}^{1-\epsilon(k)} d z \sum_{b, c} P_{a \rightarrow b c}(z) \frac{\alpha_{s}\left[z(1-z) q^{2}\right]}{2 \pi}(\$ 3)\right.
\end{aligned}
$$

This is just another form of Eq. (77) integrated over $z$. One can see that the Sudakov form factor [106] is the solution of the above equation in which unitarity plays an important role.

In principle, one can perform the simulation of initial state radiation processes in a similar way. The partons inside a nucleon can initiate a space-like branching increasing their virtuality from some initial value $Q_{0}^{2}$. A hard scattering can be considered as a probe which can only resolve partons with virtuality up to the scale of the hard scattering. Otherwise without the scattering, the off-shell partons are only virtual fluctuations inside the hadron and they will reassemble back to the initial partons. In PYTHIA, which uses backward evolution, a hard scattering is selected first with the known QCD-evolved structure function at that scale, and then the initial branching processes are reconstructed down to the initial scale $Q_{0}^{2}$. The evolution equations are essentially the same as in final state radiation except that one has to convolute with the parton structure functions [17. HIJING explicitly uses subroutines from PYTHIA to simulate each hard parton scattering and the associated initial and final state radiations. The initial virtuality for the initial state evolution is set to be $Q_{0}=2 \mathrm{GeV} / c$, and the minimum virtuality for the final state radiation is $\mu_{0}=0.5$ $\mathrm{GeV} / c$. The maximum virtuality for the associated radiations in a hard scattering with transverse momentum transfer $p_{T}$ is chosen to be $q_{\max }=2 p_{T}$. Angular ordering can also be enforced in PYTHIA to take into account the soft gluon interference [107] in the final state radiation.

After all binary collisions are processed, the scattered gluons from each nucleon 
are arranged according to their rapidities and connected to the valence quarks and diquarks of that nucleon in the collision. The rare hard scatterings of $q-\bar{q}$ pairs with opposite flavors are treated as a special case and processed as independent strings.

As studied in Refs. 108, 109, 110, large $p_{T}$ partons must propagate transversely through the whole excited matter. They will suffer both elastic and radiative energy loss. The energy loss $d E / d z$ is very sensitive to the Debye screening mass in the medium. Thus, the study of the energy loss of the produced hard jets or jet quenching can provide us with a unique probe of the dense matter. To test the sensitivity of final observables to jet quenching, we used a simple gluon splitting scheme (an effective induced radiation) in HIJING, given $d E / d z$ and the mean free path of the interaction $\lambda_{f}$. The interactions are mostly soft between both soft and hard partons in the medium. These interactions and induced radiation, in some way, mimic pre-equilibrium cascading and semi-thermalization of the produced partons.

Induced radiative energy loss is modeled in HIJING by determining first the points of final state interaction of hard partons in the transverse direction and performing a collinear gluon splitting at every point. We assume that interactions only occur with the locally comoving matter in the transverse direction. Interactions with the nuclear fragments are negligible, because the two nuclear discs pass each other on a very short time scale $2 R_{A} / \gamma \ll 1 \mathrm{fm}$. The interaction points are determined by a probability with a constant mean free path $\lambda_{f}$,

$$
d P=\frac{d \ell}{\lambda_{f}} e^{-\ell / \lambda_{f}},
$$

where $\ell$ is the distance the jet has traveled in the transverse direction after its last interaction.

Since the pre-hadronization state in HIJING is represented by connected groups (strings) of valence partons and gluons (kinks), interactions can be easily simulated by transferring a part of the parton energy, $\Delta E(\ell)=\ell d E / d z$, from one string configuration to another. Collinear gluon splitting results in a net jet quenching at the stage of hadronization, because the original hard parton energy is shared among several independent strings. This simple mechanism of course conserves energy and momentum and is numerically simple. A more dynamical parton cascade approach involving the space-time development of parton showering and collisions between the produced partons has been made in PCM [20].

To make sure that the model incorporates the right physics down to low energies we have compared the numerical results in $p A$ and $A A$ collisions with data at SPS energies. Shown in Fig. 12 are the calculated rapidity distributions of negative charged particles in $p p$ (dot-dashed histogram), $p A r$ (dashed histogram) and $p X e$ 


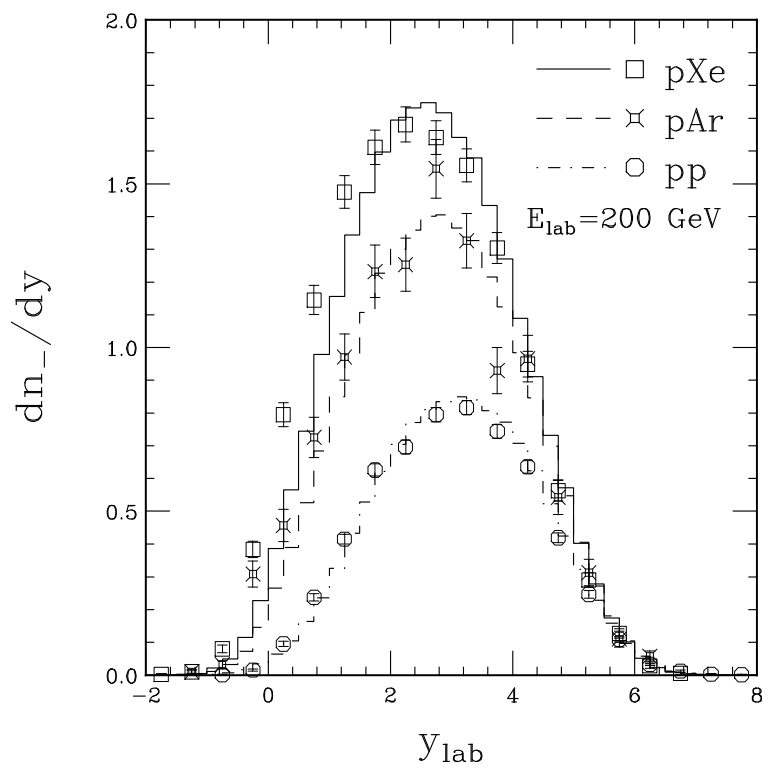

Figure 12: Rapidity distributions for negative particles in $p p$ (circle, dot-dashed histogram), $p A r$ (crossed-square, dashed histogram)and $p X e$ (square, solid histogram) collisions at $E_{\text {lab }}=200 \mathrm{GeV}$. The points are data from Ref. [111] and histograms are from HIJING calculation.

(solid histogram) collisions at $E_{\text {lab }}=200 \mathrm{GeV}$. The data are from Ref. [111. Because jet production is negligible at this energy, particle production occurs mainly through soft excitations of projectile and target nucleons. The HIJING low $p_{T}$ algorithm reproduces an increase of particle production in the central region with the number of participating target nucleons. The peak of the rapidity distribution is shifted back towards the target region and its height is proportional to the target atomic number. In the target region, HIJING under-predicts particle production due to the neglect of final state cascading.

Shown in Figs. 13 and 14 are the calculated rapidity distributions of negative particles in central $O+A u$ collisions at $E_{\text {lab }}=60$ and $200 \mathrm{AGeV}$, and the transverse momentum distributions of negative particles in $p+p$ and central $O+A u$ collisions at $E_{\text {lab }}=200 \mathrm{AGeV}$. The overall features of the data 112 are well accounted for except for the enhancement at low $p_{T}<0.2 \mathrm{GeV} / c$ in $O+A u$. That enhancement is currently believed to originate also from final state interactions [113, 114]. The data for the $O+A u$ collisions are taken with a central trigger. In HIJING simulations, central events are selected which can give the corresponding averaged multiplicity.

The calculated results for central $A u+A u$ collisions at $\sqrt{s}=200 \mathrm{AGeV}$ are shown in Fig. 15. The left panel shows the pseudorapidity distributions of charged particles. Note that without minijets (dotted), the $2 A$ soft beam jets in HIJING 


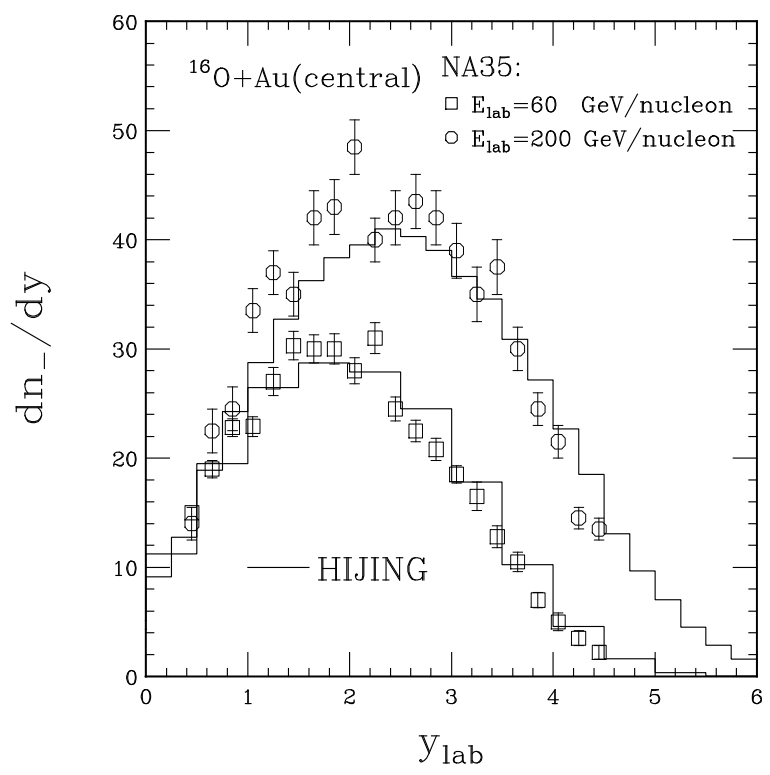

Figure 13: Rapidity distributions for negative particles in central $O+A u$ collisions at $E_{\text {lab }}=60$ and $200 \mathrm{AGeV}$. The data are from Ref. [112] and histograms are from HIJING calculation.

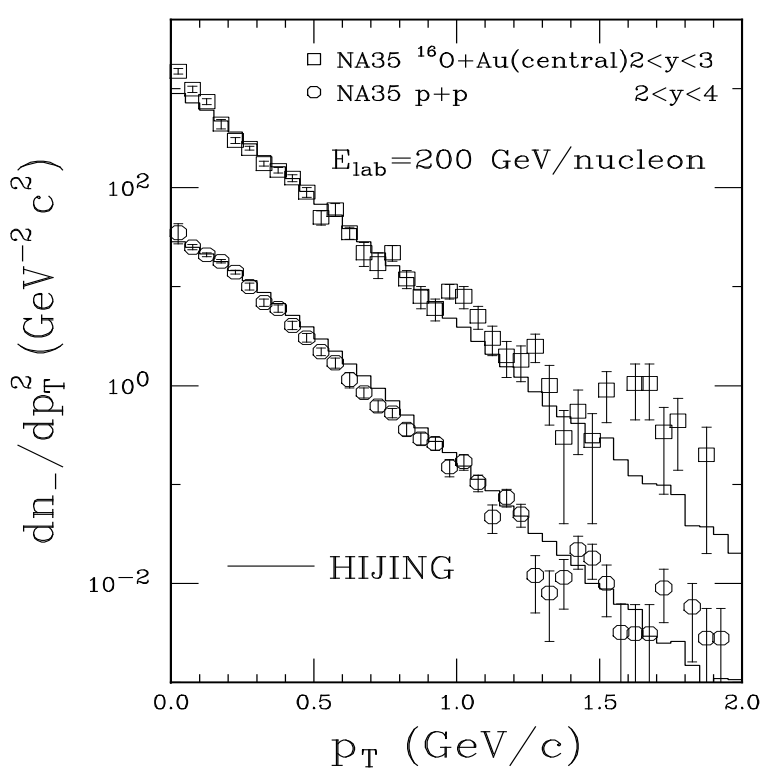

Figure 14: $p_{T}$ distributions for negative particles in $p p$ and central $O+A u$ collisions at $E_{\text {lab }}=200 \mathrm{AGeV}$. The data are from Ref. [112 and histograms are from HIJING calculation. 


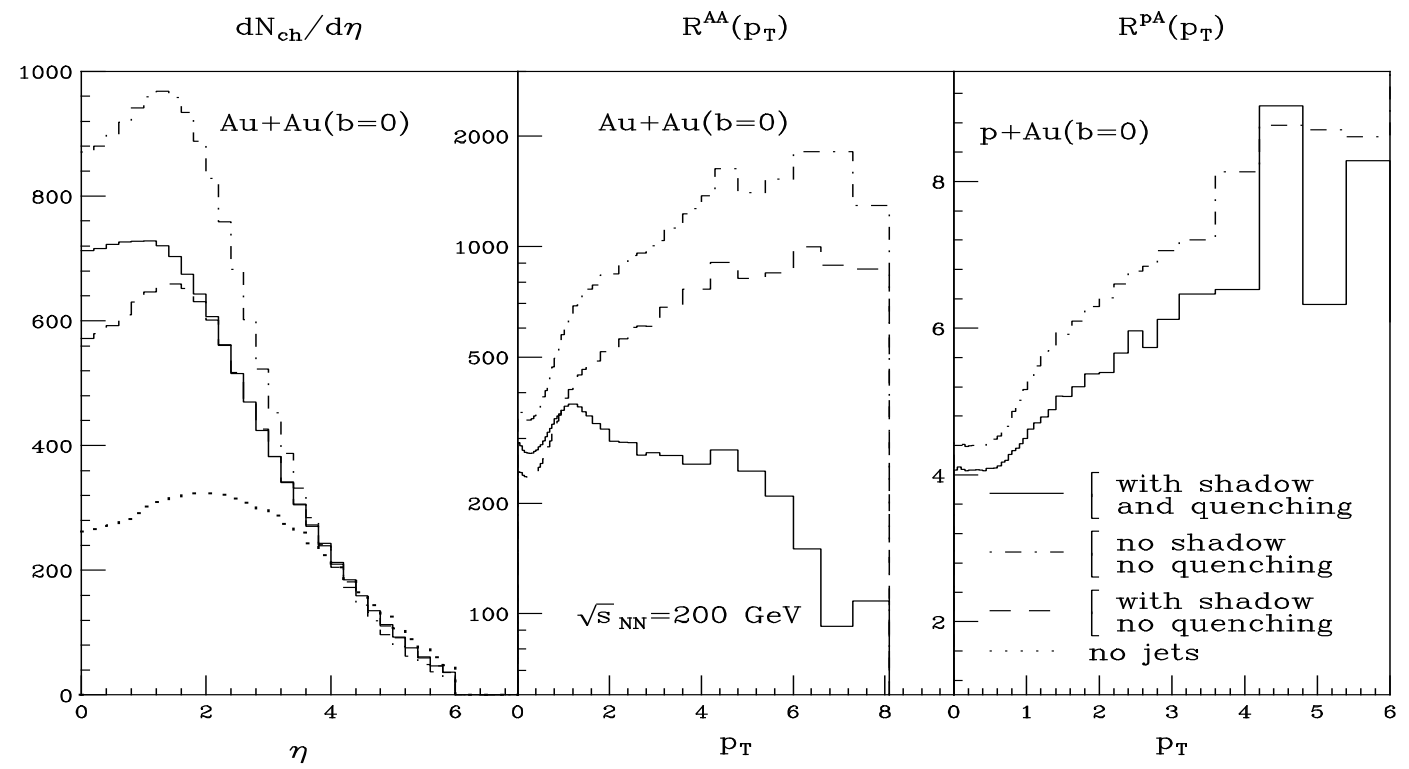

Figure 15: HIJING results of the dependence of the inclusive charged hadron spectra in central $A u+A u$ and $p+A u$ collisions on minijet production (dash-dotted), gluon shadowing (dashed), and jet quenching (solid) assuming that gluon shadowing is identical to that of quarks and $d E / d z=2 \mathrm{GeV} / \mathrm{fm}$ with $\lambda_{f}=1 \mathrm{fm} . R^{A B}\left(p_{T}\right)$ is the ratio of the inclusive $p_{T}$ spectrum of charged hadrons in $A+B$ collisions to that of $p+p$.

lead to $d N_{A A} / d \eta \approx A d N_{p p} / d \eta$. Each beam jet contributes about 0.75 to the central rapidity density almost independent of energy [74. Without gluon shadowing(dashdotted), minijets are found to approximately triple the rapidity density due to beam jets. However, if gluon shadowing is of the same magnitude as that for quarks and antiquarks, then the minijet contribution to the rapidity density is reduced by approximately a half (dashed). The solid histogram shows that the effect of jet quenching on the rapidity density is small for $d E / d z=2 \mathrm{GeV} / \mathrm{fm}$ and $\lambda_{f}=1 \mathrm{fm}$.

Plotted in the middle panel of Fig. 15, is the ratio,

$$
R^{A B}\left(p_{T}\right)=\frac{d^{2} N_{A B} / d p_{T}^{2} / d \eta}{d^{2} N_{p p} / d p_{T}^{2} / d \eta}
$$

of the inclusive $p_{T}$ spectrum of charged particles in central $A u+A u$ collisions to that of $p+p$. For $p_{T}>2 \mathrm{GeV} / c$, both shadowing and quenching are seen to reduce dramatically the inclusive hadron production. In the absence of shadowing and jet quenching (dash-dotted) the ratio approaches the number of binary $p p$ collisions in the reaction. Shadowing alone (dashed) suppresses moderate $p_{T}$ hadrons by a factor of about 2. Inclusion of jet quenching with $d E / d z=2 \mathrm{GeV} / \mathrm{fm}$ reduces that yield by 
another factor of about 3-5. It is remarkable and encouraging that the single inclusive hadron spectrum appears so sensitive to nuclear effects on jet production. Clearly higher order correlations, e.g., high $p_{T}$ back-to-back hadron pairs, will be even more sensitive to these effects.

From the above, however, we see that $A+A$ data alone would not be sufficient to disentangle the effects of shadowing and jet quenching. To separate the two, $p+A$ collisions must be studied at the same energy! In those reactions, the density of comoving hadrons is so low that final state interactions leading to jet quenching should be negligible. Therefore, any observed suppression of moderate $p_{T}$ hadrons could be attributed to gluon shadowing alone. The right panel in Fig. 15 shows the ratio as defined in Eq. (85) for central $p+A u$ collisions. Without shadowing (dash-dotted), the high $p_{T}$ limit again reaches the number of binary collisions, which is about 8 in this case. The low $p_{T}$ limit is, on the other hand, controlled by the number of pairs of beam jets, which is approximately 4 in central collisions. We see from this figure that the ratio $R^{p A}\left(p_{T}\right)$ at moderate $p_{T}$ is indeed sensitive to gluon shadowing. Therefore, a systematic measurement of the inclusive hadron spectra in $p+p$ and $p+A$ at the same energy is essential in order to determine the magnitude of gluon shadowing. Since the Cronin effect on jet production in $p+A$ collisions at $\sqrt{s} \gtrsim 100 \mathrm{AGeV}$ is expected to be small, the spectra of moderate $p_{T}$ hadrons will provide information on the gluon structure function complementary to that deduced by the conventional direct photon technique that is limited to higher $x$ gluons due to the high $\pi^{0} \rightarrow \gamma \gamma$ background. Once the magnitude of gluon shadowing is determined, its contribution to the observed suppression of moderate $p_{T}$ hadrons in $A+A$ data can be calculated, thereby making it possible to isolate the the contribution due to jet quenching.

\subsection{Space-Time Structure of Parton Production}

\subsubsection{Space-time history}

So far I have discussed the general features of a pQCD-inspired model for parton and particle production in hadronic and nuclear collisions. However, in order to estimate the initial number and energy density of the produced partons, one must know their formation time. The total formation time must include the interaction time of the semihard processes and the formation time for initial and final state radiations. As demonstrated in Refs. [26, 27], formation time is a consequence of interference in multiple scattering processes, which can also be obtained from the uncertainty principle. Destructive interference suppresses those secondary scatterings which happen within the formation time of the previous interactions. Inclusion of the formation time effects is necessary to treat the secondary scatterings properly. Otherwise, one might 
overestimate the interaction rates and parton production. One source of overestimate may arise if one neglects the interaction time of the scatterings. During the interaction time, which depends on whether the scattering is hard, semihard or soft, the participating and therefore the produced partons cannot scatter again immediately with the beam partons. Another overestimate has to do with the initially radiated partons before the hard or semihard scatterings. In calculating jet cross sections and the number of hard scatterings, parton structure functions of a nucleus $f_{a / A}\left(x, Q^{2}\right)$ evaluated at the scale $Q^{2}=p_{T}^{2}$ are used. These parton distribution functions then have already included the effect of QCD evolution, producing more partons at small $x$ and larger $Q^{2}$. Therefore, in Monte Carlo simulations in which initial radiations are treated by backward evolution, the radiated partons should not participate in any interactions before the end of the corresponding hard scattering. This is also in accord with the factorization theorem [115] of pQCD.

Formation time is usually related to a radiative process. According to the uncertainty principle, an off-shell parton can be considered as a virtual fluctuation and it can only live for a finite time, $\Delta t$, determined by its virtuality $q^{2}$,

$$
\Delta t \approx q_{0} / q^{2},
$$

where $q_{0}$ is the energy of the parton. This $\Delta t$ is exactly the formation time of the radiation which will follow. Thus, after $\Delta t$, the off-shell parton will then branch or "decay" into other partons which can further initiate branchings until a minimum virtuality $\mu_{0}$ is reached. At $q^{2} \leq \mu_{0}^{2}$, pQCD is not considered to be valid anymore and the process of nonperturbative hadronization takes over. Following this tree of branching (which also includes initial space-like radiation) and assuming a classical straight line trajectory for partons, one can then follow the space and time evolution of the initial parton production 116.

The life time of a virtual parton in Eq. (86) is only a rough estimate according to the uncertainty principle. One could also estimate it via a pQCD analysis of the radiative amplitudes induced by multiple scatterings [27, 31]. Thus, $q_{0} / q^{2}$ should be a good estimate of $\Delta t$ in magnitude. However, this formation time or the life time of a virtual parton cannot be identified with the decay rate of a virtual parton as has been done in PCM [20] because the later has an additional dependence on $\alpha_{s}$. The decay rate is only related to the radiation intensity, not the formation time of the radiated partons.

Eq. (86) can also be used to estimate the interaction time for each hard scattering with $q$ being the sum of the initial or final four-momenta of the colliding partons. If the fractional momenta of the partons are $x_{1}$ and $x_{2}$, then the interaction time can 
be estimated as

$$
\Delta t_{i} \approx \frac{x_{1}+x_{2}}{2 x_{1} x_{2} \sqrt{s}}
$$

In this case, the asymmetric scatterings $\left(x_{1} \gg x_{2}\right.$ or $\left.x_{1} \ll x_{2}\right)$ have longer interaction time than the symmetric ones $\left(x_{1} \sim x_{2}\right)$ for fixed parton-parton center-of-mass energy $x_{1} x_{2} \sqrt{s}$. We will also assume that the interaction time is the same for all channels.

In the rest frame of each nucleus, three-parameter Woods-Saxon nuclear densities are used to construct the nucleon distribution inside the nucleus. The system is then boosted to the center-of-mass frame of the two colliding nuclei. Due to the fact that gluons, sea quarks and antiquarks are only quantum fluctuations before they really suffer scatterings, their longitudinal distribution around the center of the nucleon is still governed by the uncertainty principle in any boosted frame [117]. One usually refers to this distribution as the "contracted distribution", in which a parton with $x_{i}$ fractional momentum has a finite spatial spread,

$$
\Delta z_{i} \approx 2 / x_{i} \sqrt{s}
$$

Transversely, partons are distributed around their parent nucleons according to the Fourier transform of a dipole form factor [35. If we define $t=0$ as the moment when the two nuclei have complete overlap, the overlap of two colliding nucleons can happen anywhere inside the diamond-shaped region (with dotted lines) in Fig. 16. The diagonal length of the diamond is $2 R_{A} \gamma$ with $\gamma$ the Lorentz boost factor. The interaction point of two partons in a $t-z$ plane can be anywhere within the shaded area in Fig. 16. The solid lines are the trajectories of the two parent nucleons which spread around the nuclei according to a longitudinally contracted Woods-Saxon distribution.

To study the space-time history of parton production, let us look at the results of HIJING simulations for $A u+A u$ at the highest RHIC energy, $\sqrt{s}=200 \mathrm{AGeV}$. Shown in Fig. 17 is the total number of produced partons, on-shell as well as offshell, as a function of time. We see that long before the two nuclei overlap and hard scatterings take place, partons have already been produced via initial state radiation. Note that, if the coherence is not broken by the hard scattering, partons which would have been emitted from the initial state radiation will not emerge as produced partons. In Fig. 17 the initiators of the space-like branching are also included as produced partons. Therefore, a parton is defined to be a produced one before the hard scattering if it has initial state radiation. If a parton does not have initial state radiations, it will only become a produced parton after the hard scattering. From Fig. 17, we can see that about $2 / 3$ of the total number of partons are produced between $t=-0.5$ and $0.5 \mathrm{fm} / c$ while about 200 semihard scatterings happen between $t=-0.1$ and 0.1 $\mathrm{fm} / \mathrm{c}$ as indicated by dashed lines. We find also that about $2 / 3$ of the total number 


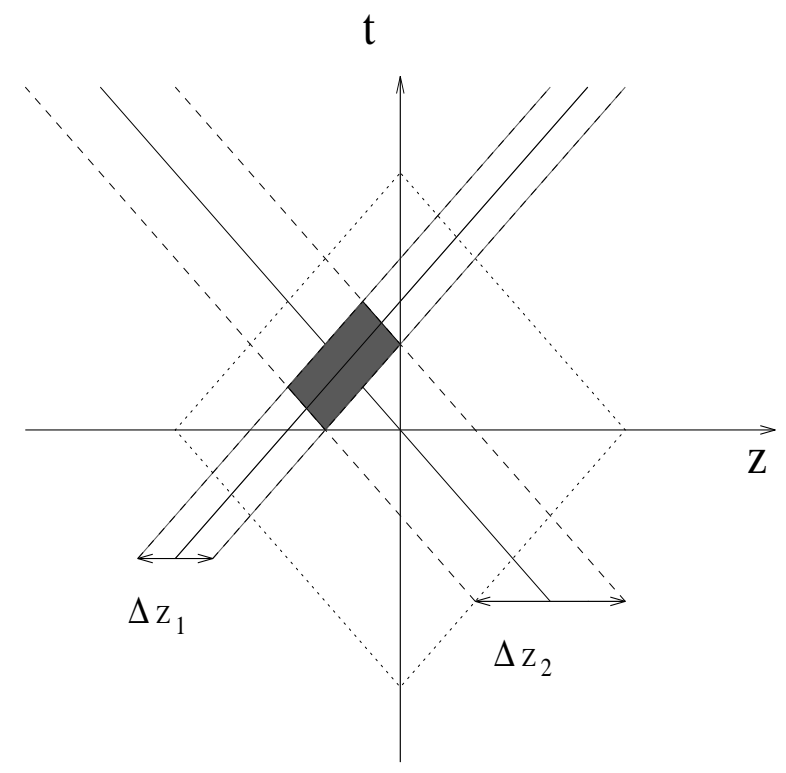

Figure 16: The overlap region in space and time of two incoming partons each with spatial spread of $\Delta z_{1}$ and $\Delta z_{2}$, respectively. The interaction point is chosen randomly inside the shaded region. Solid lines show the trajectories of the parent nucleons and dotted lines represent the overlap region of the two nuclei.

of partons are produced in initial and final state radiations. The fraction of partons from branching should increase with the colliding energy and with smaller choices for $\mu_{0}$.

To see how hard or semihard scatterings and initial and final state bremsstrahlung contribute to parton production, the rapidity distribution of produced partons at different time is shown in Fig. 18. Before $t=0$, most of the partons come from initial state radiation. Since the radiations are almost collinear, these partons move along the beam direction and therefore have large rapidities. The semihard scatterings then produce partons uniformly over a rapidity plateau and fill up the middle rapidity region. Final state radiations, which happen after the semihard scatterings, will also produce partons uniformly in the central rapidity region. It is quite clear from Fig. 18 that the dip of $d N / d y$ in middle rapidity is actually caused by the parton production from initial state bremsstrahlung at large rapidity. These partons with large longitudinal momenta will move away from the interaction region after the semihard scatterings. They do not rescatter with the beam partons in the leading twist approximation.

Fig. 19 contains snapshots of $d N / d z$ at different times to illustrate how the parton production evolves in space and time. At $t=-0.7 \mathrm{fm} / c$, as the two nuclei approach toward each other before they actually overlap, initial state radiations have 


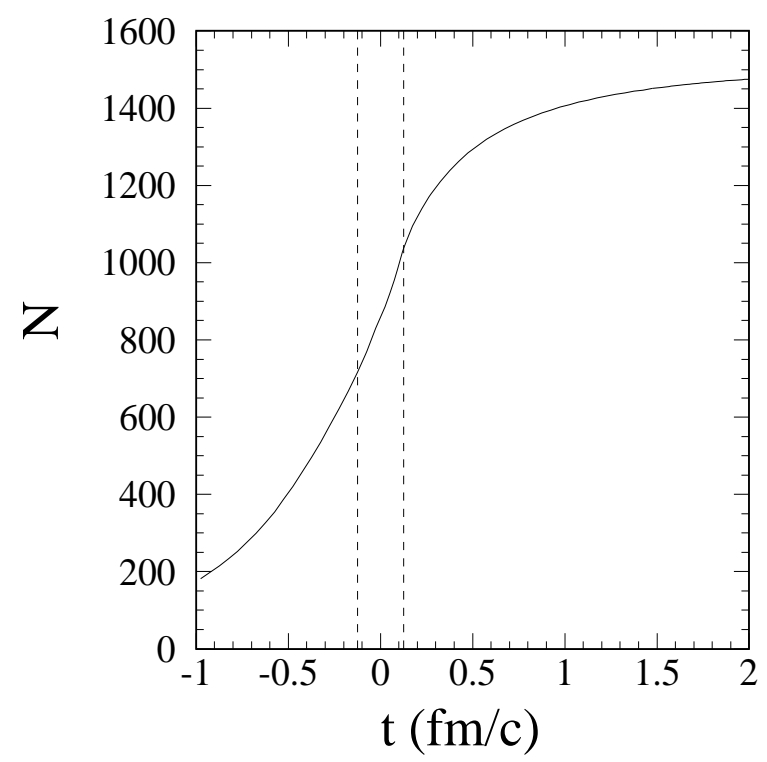

Figure 17: The total number of produced partons $N$ as a function of time $t$, with $t=0$ defined as when the two nuclei have complete overlap.

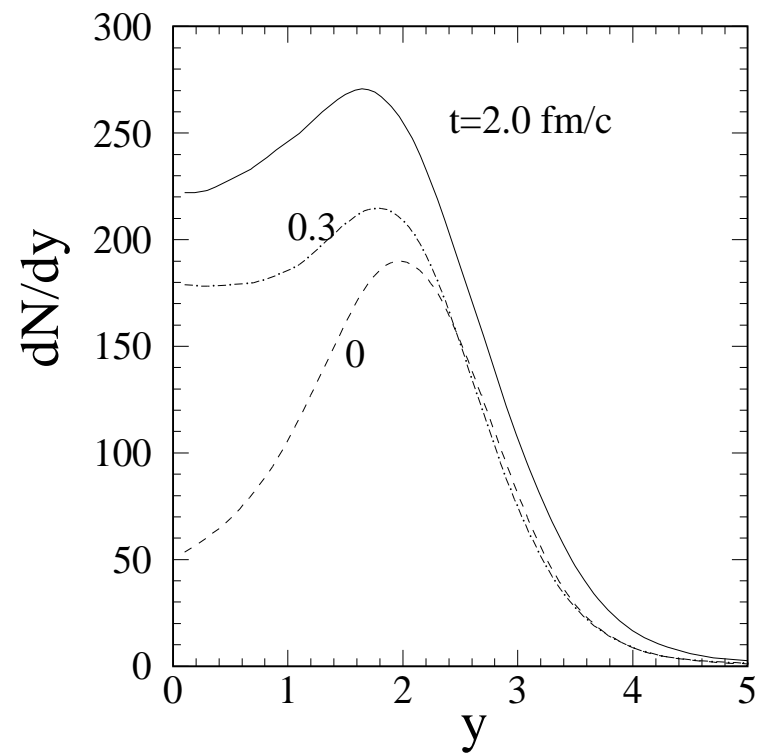

Figure 18: The rapidity distribution $d N / d y$ of produced partons at different times $t$. 


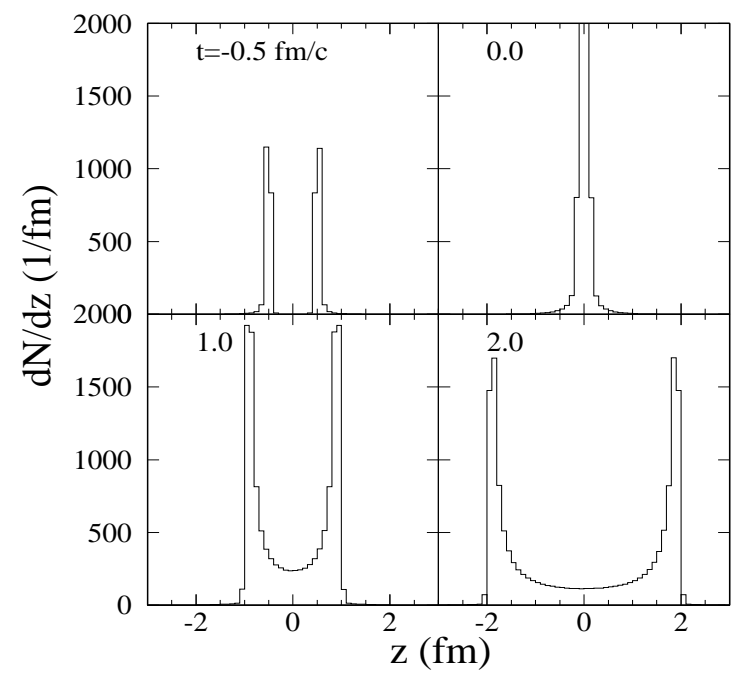

Figure 19: Parton distribution along the $z$-axis at different times.

already begun. These partons have large rapidities and are Lorentz contracted with an average spread in $z$,

$$
\Delta z \approx 1 / p_{0}+2 R_{A} \frac{2 m_{N}}{\sqrt{s}} \approx 0.25 \mathrm{fm}, \sqrt{s}=200 \mathrm{GeV},
$$

where $p_{0}=2 \mathrm{GeV} / c$ is the $p_{T}$ cutoff for semihard scattering, $R_{A}$ is the nuclear radius of $A u$ and $m_{N}$ is the nucleon mass. After the hard scatterings and during the interaction time, partons are produced uniformly in the central rapidity region. Afterwards, partons follow straight lines by free-streaming and are distributed evenly in $z$ between the two receding pancakes of beam partons (partons from initial state radiations). The hyperbolic-shaped equal-time parton distribution in $z$ corresponds approximately to a boost invariant parton density in the central region in this freestreaming picture.

Another illustrative way to study the evolution of local parton density is to make a contour plot of parton density $n$ in $z$ and $t$ as shown in Fig. 20. Here, $n$ is defined as

$$
n=\frac{1}{\pi R_{A}^{2}} \frac{d N}{d z},
$$

and a sharp sphere distribution is assumed for the nuclear density. One can clearly see that partons inside the two approaching nuclei have a spatial spread of $\Delta z=0.25$ $\mathrm{fm}$ in $z$. This spread continues for partons from the initial state radiation as they escape from the interaction region along the beam direction with large rapidities. The 


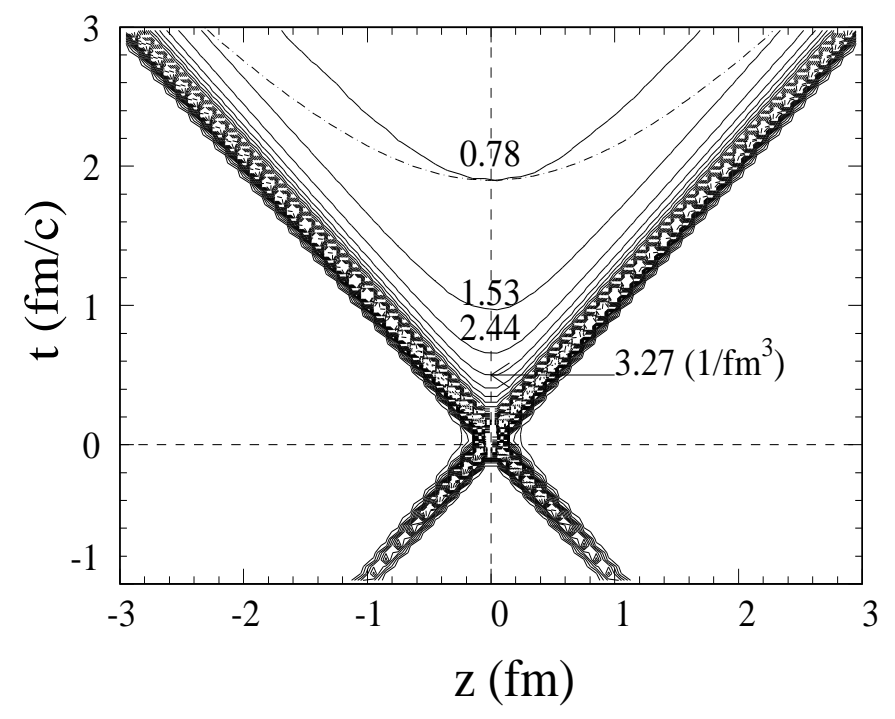

Figure 20: Contour plot in $z-t$ plane of the parton density $n$ of Eq. (90), as indicated by the numbers. The wavy structure along the light-cone is only an artifact of the plotting program.

interaction region where semihard scatterings happen lasts for about $0.5 \mathrm{fm} / c$, from $t=-0.25$ to $0.25 \mathrm{fm} / c$. Because of the contracted distribution, some of the partons lie outside the light-cone which is defined with respect to the overlapping point of the two nuclei.

If one assumes boost invariance [118], the parton density can be estimated as

$$
n=\frac{1}{\pi R_{A}^{2} \tau} \frac{d N}{d y},
$$

where $\tau$ is the proper time and $n$ should be a function of $\tau$ only. By comparing the contour of constant density with the hyperbola of constant $\tau$ (dot-dashed line) in Fig. 20, we see that this is approximately true. We also see that the density, as indicated by the numbers, decreases like $1 / \tau$ due to free-streaming.

\subsubsection{Local isotropy and chemical composition}

Since, as we have demonstrated, there are numerous partons produced within a rather short time, the initial parton density is very high at $\tau \geq 0.25 \mathrm{fm} / c$, immediately after the interaction region (see Fig. 20). Within such a dense system, secondary parton scatterings and production are inevitable at a time scale much larger than the average formation time. The equilibration time for the system can be estimated by solving a 
set of rate equations [119] as I will show later. In this approach, one must make sure that there is approximately local isotropy in momentum space. This could be achieved through secondary parton scatterings as has been investigated in Refs. [20, 120]. One can also use free-streaming to estimate the upper bound of the thermalization time [119, 121], by studying the momentum distribution of partons in a cell of the size of the mean free path $\lambda_{f}$, assuming that parton scatterings are frequent enough to achieve thermalization within this upper bound.

Let's concentrate on the central slice at $z=0$ with $|z|<0.5 \mathrm{fm}$. At the very early stage during the interaction region, produced partons with different rapidities are confined to a highly compressed slab with $\Delta z \approx 0.5 \mathrm{fm}$. As the system expands, partons with large rapidities will escape from the central slice while partons with small rapidities remain. Shown in Fig. 21 is the evolution of momentum distributions in $p_{x}$ (solid lines) and $p_{z}$ (dashed lines) at different times (indicated by the number on each line). The slope of the $p_{z}$ distribution decreases because partons with large longitudinal momenta gradually escape from the central region. At $t=0.7 \mathrm{fm} / c$, the slopes of $p_{x}$ and $p_{z}$ distributions become the same. We should note that the isotropy in momentum distribution via free-streaming at this moment is only a transient phenomenon which will disappear quickly afterwards. What really determines the thermalization time is the relaxation time due to parton scatterings [122, 123]. If the relaxation time is short, thermalization can be achieved even before the transient momentum isotropy. On the other hand, for a longer relaxation time, the momentum distribution will become anisotropic again and will eventually approach thermal equilibration afterwards. To calculate the relaxation time, one has to consider both elastic [122] and inelastic [123 parton scatterings and include resummation of hot thermal loops. Unfortunately, a complete calculation including higher order processes in a nonequilibrium system is not available. In the following we will simply assume that the time for local momentum isotropy can be replaced by the time for the central slab of about $1 \mathrm{fm}$ to achieve isotropy in momentum space via free-streaming,

$$
\tau_{\text {iso }} \approx 0.7 \mathrm{fm} / c
$$

The readers should be reminded that such assumption will give rise to corrections from the neglected viscosities to the results concerning parton equilibration.

Unlike quarks and gluons in an ideal gas with chemical equilibrium, partons produced in the initial stage of heavy-ion collisions, which are determined by the parton structure functions, the hard scattering cross sections and the radiation processes in pQCD, are far from chemical equilibrium. Due to the difference in the numbers of degrees of freedom in color space, cross sections involving gluons are always larger 


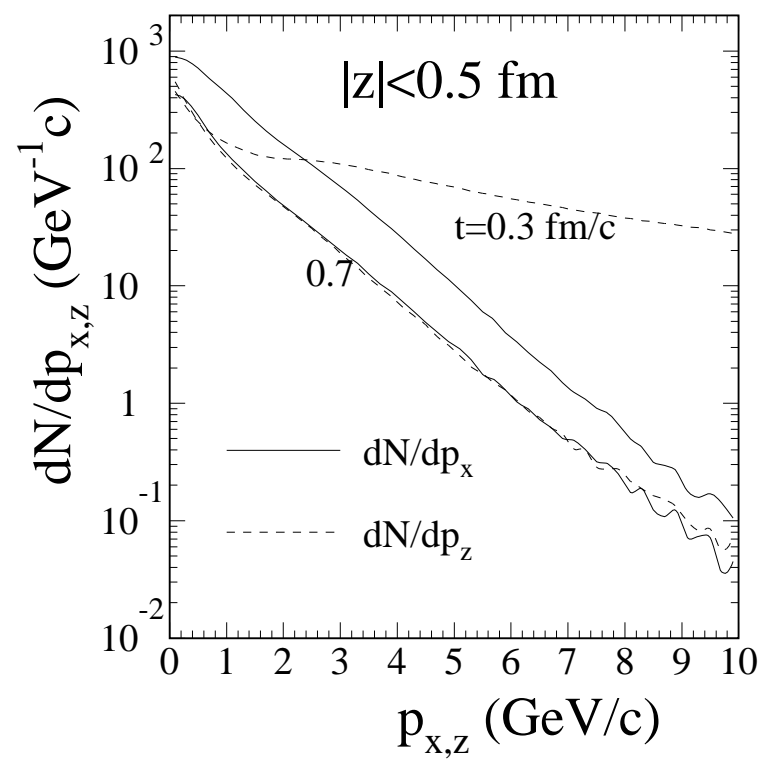

Figure 21: $p_{x}$ and $p_{z}$ distributions at different times for partons in the central slice $|z|<0.5 \mathrm{fm}$.

than those of quarks. Similarly, both initial and final state radiations produce more gluons than quarks and anti-quarks. Therefore in $\mathrm{pQCD}$, the ratio of produced quarks to gluons is much smaller than the ratio in an ideal gas, which is $9 / 4$ for three quark flavors.

Shown in Fig. 22 are the fractions of produced quarks and anti-quarks as functions of time $t$ in $p p$ and $A A$ collisions at $\sqrt{s}=200 \mathrm{AGeV}$. For $A u+A u$ collisions, hard scatterings with $p_{T}>2 \mathrm{GeV} / c$ produce about $13 \%$ quarks and anti-quarks. If initial and final state radiations are not included the ratio jumps to $18 \%$, because radiations produce more gluons than quarks and antiquarks. In $p p$ collisions, about $28 \%$ of the partons produced via hard scatterings without radiations are quarks and anti-quarks.

The difference between $A u+A u$ and $p p$ collisions is due to the different $A$ dependence of the valence and sea quark production. Since the partons are produced via binary collisions, the number of produced gluons and sea quarks and antiquarks scales like $A^{4 / 3}$. On the other hand, baryon number conservation requires valence quark production to scale like $A$. Given the fraction of total produced quarks $q_{p p}=$ 0.28 and valence quarks $v_{p p}=0.14$ in $p p$ collisions, one can find for $A u+A u$ collisions,

$$
q_{A A}=\frac{q_{p p}-\left(1-A^{-1 / 3}\right) v_{p p}}{1-\left(1-A^{-1 / 3}\right) v_{p p}},
$$




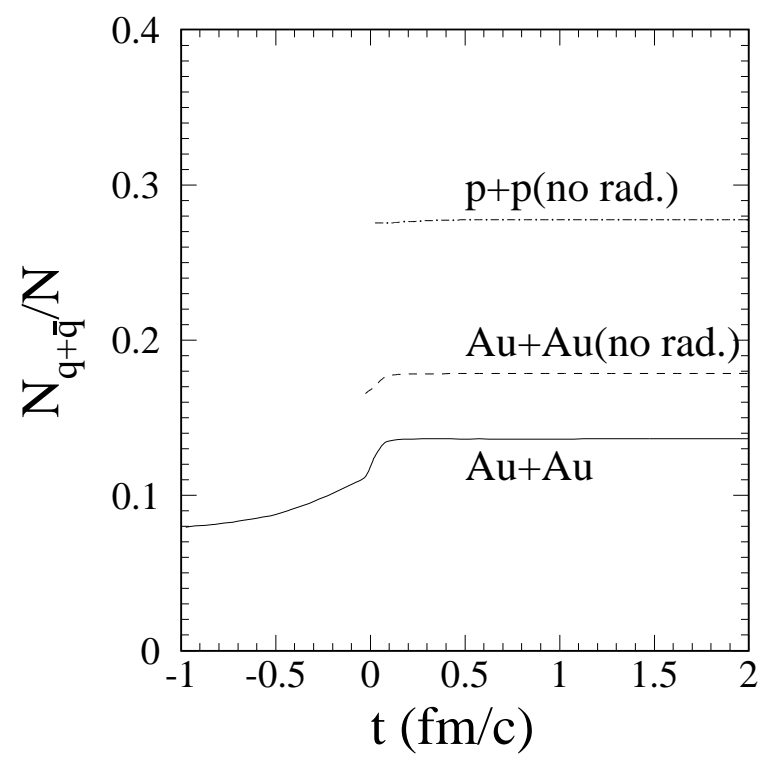

Figure 22: The fractional number of produced quarks and anti-quarks as a function of time for $A u+A u$ (solid)collisions, $A u+A u$ without radiations (dashed), and $p+p$ without radiation (dot-dashed).

which gives $q_{A A}=0.18$ for $A=197$, as we obtained from Fig. 22 of the numerical calculation. To demonstrate the effective valence quark production, we plot in Fig. 23 the rapidity dependence of the fractional quark number. In $p p$ collisions, the valence quark production peaks at large rapidities. In nucleus-nucleus collisions, the valence quark production has a different scaling in $A$ than gluons and sea quarks. This is why the fraction of quark and antiquark production is suppressed more at large rapidity than in the central rapidity region. The relative quark and antiquark production in the central rapidity region is further suppressed by final state radiation.

The relative quark production found in PCM [124] is about five times larger than what I show here. This is due to an overestimate of intrinsic quark production through flavor excitations in PCM. Such flavor excitations should be suppressed by interference among pQCD amplitudes at the same order [125]. For the same reason, other heavy quark production is also overestimated in PCM [126].

The small number of produced quarks relative to gluons within pQCD has important consequences for chemical equilibration of the partonic system. Because of the small initial relative quark density and small quark production cross section as compared to gluon production, it takes a very long time for the system to achieve chemical equilibrium, if it ever does. If this time is longer than the phase transition time, a fully equilibrated QGP may never be formed [119]. 


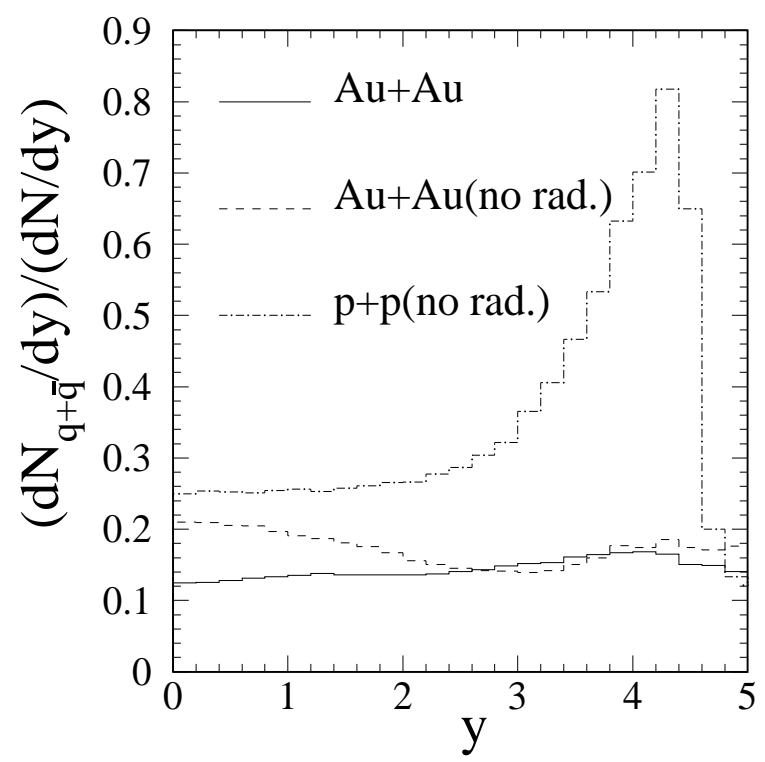

Figure 23: The final fractional number of produced quarks and anti-quarks as functions of rapidity for $A u+A u$ (solid)collisions, $A u+A u$ without radiations (dashed), and $p+p$ without radiation (dot-dashed).

\subsubsection{Multiple initial parton scatterings?}

I have already demonstrated earlier in Sec. 3.2 that multiple parton scatterings become less important at high energies and eventually will disappear. This phenomenon can also be understood in a space-time picture. But before doing that, I should emphasize again that the multiple parton scatterings in this context always refer to those during the overlapping time of two colliding nuclei. They are different from the final parton rescatterings which are responsible for parton thermalization.

We have explicitly taken into account the interaction time for the semi-hard scatterings, which is roughly $\tau_{i} \sim 1 / p_{T}$. Inside a highly Lorentz contracted nucleus, the spatial spread for partons which could participate in a hard scattering with transverse momentum transfer $p_{T}$ is also about $\Delta z \sim 1 / p_{T}$. This would leave the produced partons no time to have another hard scattering of $p_{T}$ with the incoming beam partons. For finitely contracted nuclei at relatively low energies, this kind of double high $p_{T}$ scattering is still possible. However, these higher twist processes should be suppressed by a factor of $1 / p_{T}^{2}$. It is also possible for a parton to go through a hard and a soft scattering subsequently since the soft partons always have a spatial spread of $1 \mathrm{fm}$. This kind of hard-soft multiple interactions constitutes the leading contribution to higher twist corrections to hard processes in nuclear collisions [127]. However, double semihard scatterings at high energies with $p_{T} \sim p_{0}$ will be suppressed due to 
finite interaction time.

The large $p_{T}$ enhancement of both Drell-Yan dilepton production and single hadrons in $p A$ collisions at $\sqrt{s} \leq 50 \mathrm{GeV}$ is considered as a result of multiple parton interactions [128]. At these energies, the contracted length of a heavy nucleus is still relatively larger than the interaction and formation time. The factorized form of the parton model is then modified due to the finite beam energy. However, a collection of experimental data [86, 103] even in this intermediate energy range has already shown the effects of the finite interaction time. As the energy increases, the interaction time becomes more important as compared to the size of an increasingly contracted nucleus. The partons then have less time for secondary scatterings. This then leads to the observed decrease of the large $p_{T}$ enhancement in $p A$ interactions as

the beam energy increases. At ultrarelativistic energies, one should therefore expect the suppression of multiple semihard scatterings. As I have argued earlier, partons from the associated initial state radiation will not scatter again with the incoming beam partons due to factorization.

\section{Parton Equilibration}

The initial semihard interactions between two beams of partons happen in a short period of time. After the leading partons leave the interaction region, a partonic system will be formed in the central region. In this section, I will discuss how this partonic system equilibrates and describe the physics involved, based on a microscopical description. The thermalization and equilibration in this description are driven by multiple scatterings and parton production (or annihilation) by induced radiation (or absorption). Though an exact quantum field treatment of multiple parton interaction is not possible with present technology, a semiclassical approximation has been undertaken [20]. The most important and difficult effort in a semiclassical approximation is to simulate the quantum effects, like interference, coherence, and especially, nonperturbative phenomena in QCD. Under extreme conditions at high temperature and densities, these quantum effects become very important and may dictate the evolution of the partonic system. In this section, I will also discuss the interference effects associated with induced radiations, since radiative processes produce extra partons, leading to fast parton equilibration. 


\subsection{Initial Conditions: a Hot and Undersaturated Gluonic Gas}

As we have discussed earlier, the kinematic separation of partons with different rapidities in a central slab of about $1 \mathrm{fm}$ give rise to a transient local momentum isotropy at the time of the order of $\tau_{\text {iso }}=0.7 \mathrm{fm} / c$. We will further assume this is the actual kinetic equilibration (or thermalization) time for the partonic system. Though this assumption is not well founded, this is the best we can do before a complete calculation of the relaxation time due to parton rescatterings and radiations is available. After this time the momentum distribution of partons is assumed to be locally isotropic and approximately exponential. What we are interested next is how this system approaches chemical equilibrium.

Let us first estimate the initial conditions for the equilibration from the previous chapters. Since we are here primarily interested in the chemical equilibration of the parton gas, we shall assume that the parton distributions can be approximated by thermal phase space distributions with non-equilibrium fugacities $\lambda_{i}$ :

$$
f\left(k ; T, \lambda_{i}\right)=\lambda_{i}\left(e^{\beta u \cdot k} \pm \lambda_{i}\right)^{-1}
$$

where $\beta$ is the inverse temperature and $u^{\mu}$ is the four-velocity of the local comoving reference frame. When the parton fugacities $\lambda_{i}$ are much less than unity as may happen during the early evolution of the parton system, we can neglect the quantum statistics in Eq. (94) and write the momentum distributions in the factorized form,

$$
f\left(k ; T, \lambda_{i}\right)=\lambda_{i}\left(e^{\beta u \cdot k} \pm 1\right)^{-1}
$$

Using this form for the distributions, one has the parton and energy densities,

$$
n=\left(\lambda_{g} a_{1}+\lambda_{q} b_{1}\right) T^{3}, \quad \varepsilon=\left(\lambda_{g} a_{2}+\lambda_{q} b_{2}\right) T^{4}
$$

where $a_{1}=16 \zeta(3) / \pi^{2} \approx 1.95, a_{2}=8 \pi^{2} / 15 \approx 5.26$, for a Bose distribution, $b_{1}=$ $9 \zeta(3) N_{f} / \pi^{2} \approx 2.20$ and $b_{2}=7 \pi^{2} N_{f} / 20 \approx 6.9$ for a Dirac distribution for a baryon symmetric system, $\lambda_{q}=\lambda_{\bar{q}}$. Since we have demonstrated that boost invariance is a good approximation for the initially produced partons, we can then estimate the initial parton fugacities, $\lambda_{g, q}^{0}$ and temperature $T_{0}$ from [cf. Eq. (91)]

$$
n_{0}=\frac{1}{\pi R_{A}^{2} \tau_{\text {iso }}} \frac{d N}{d y}, \quad \varepsilon_{0}=n_{0} \frac{4}{\pi}\left\langle k_{T}\right\rangle,
$$

where $\left\langle k_{T}\right\rangle$ is the average transverse momentum. The quark fugacity is taken as 


\begin{tabular}{lcc}
\hline \hline & RHIC & LHC \\
\hline$\tau_{\text {iso }}(\mathrm{fm} / c)$ & 0.7 & 0.5 \\
$\varepsilon_{0}\left(\mathrm{GeV} / \mathrm{fm}^{3}\right)$ & 3.2 & 40 \\
$n_{0}\left(\mathrm{fm}^{-3}\right)$ & 2.15 & 18 \\
$\left\langle k_{T}\right\rangle(\mathrm{GeV})$ & 1.17 & 1.76 \\
$T_{0}(\mathrm{GeV})$ & 0.57 & 0.83 \\
$\lambda_{g}^{0}$ & 0.05 & 0.124 \\
$\lambda_{q}^{0}$ & 0.008 & 0.02 \\
\hline \hline
\end{tabular}

Table 1: Values of the relevant parameters characterizing the parton plasma at the moment $\tau_{\text {iso }}$, when local isotropy of the momentum distribution is first reached.

$\lambda_{q}^{0}=0.16 \lambda_{g}^{0}$, corresponding to a ratio 0.14 of the initial quark(antiquark) number to the total number of partons. Table 1 shows these relevant quantities at the moment $\tau_{\text {iso }}$, for $\mathrm{Au}+\mathrm{Au}$ collisions at RHIC and LHC energies. One can observe that the initial parton gas is rather hot, reflecting the large average transverse momentum. However, the parton gas is very far from saturation in phase space as compared to the ideal gas at the same temperature. The gas is also far away from chemical equilibrium with respect to parton species (gluons and quarks with different flavors) since it is dominated by gluons. I should emphasize that the initial conditions listed here result from HIJING calculation of parton production through semihard scatterings. Soft partons, e.g., due to parton production from the color field [61], are not included.

\subsection{Master Rate Equations}

In general, chemical reactions among partons can be quite complicated because of the possibility of initial and final-state gluon radiations. As we have discussed earlier, interference effects due to multiple scatterings inside a dense medium lead to a strong suppression of soft gluon radiation. One lesson we learned from the LPM effect is that the radiation between two successive scatterings is the sum, on the amplitude level, of both the initial state radiation from the first scattering and the final state radiation from the second one. Since the off-shell parton is space-like in the first amplitude and time-like in the second, the picture of a time-like parton propagating inside a medium in the parton cascades simulations [20] breaks down. Instead, we shall here only consider processes where a single additional gluon is radiated, such as $g g \rightarrow g g g$ between two scatterings, in which we can include the LPM effect by a radiation suppression factor. The analysis of QCD LPM effect in Refs. [27, 31] has been done for a fast parton traveling inside a parton plasma. We will use the 
results here for radiation off thermal partons whose average energy is about $T$, since we expect the same physics to happen.

In order to permit the approach to chemical equilibrium, the reverse process, i.e., gluon absorption, has to be included as well. This is easily achieved by making use of detailed balance. Closer inspection shows that gluon radiation is dominated by the process $g g \rightarrow g g g$, because radiative processes involving quarks have substantially smaller cross sections in pQCD, and quarks are considerably less abundant than gluons in the initial phase of the chemical evolution of the parton gas. Here we are interested in understanding the basic mechanisms underlying the formation of a chemically equilibrated quark-gluon plasma, and the essential time-scales. We hence restrict our considerations to the dominant reaction mechanisms for the equilibration of each parton flavor. These are just four processes [129]:

$$
g g \leftrightarrow g g g, \quad g g \leftrightarrow q \bar{q}
$$

Other scattering processes ensure the maintenance of thermal equilibrium $(g g \leftrightarrow$ $g g, g q \leftrightarrow g q$, etc.) or yield corrections to the dominant reaction rates $(g q \leftrightarrow q g g$, etc.).

Restricting ourselves to the reactions Eq. (98), the evolution of the parton densities is governed by the master equations:

$$
\begin{aligned}
& \partial_{\mu}\left(n_{g} u^{\mu}\right)=\frac{1}{2} \sigma_{3} n_{g}^{2}\left(1-\frac{n_{g}}{\tilde{n}_{g}}\right)-\frac{1}{2} \sigma_{2} n_{g}^{2}\left(1-\frac{n_{q}^{2} \tilde{n}_{g}^{2}}{\tilde{n}_{q}^{2} n_{g}^{2}}\right), \\
& \partial_{\mu}\left(n_{q} u^{\mu}\right)=\partial_{\mu}\left(n_{\bar{q}} u^{\mu}\right) \frac{1}{2} \sigma_{2} n_{g}^{2}\left(1-\frac{n_{q}^{2} \tilde{n}_{g}^{2}}{\tilde{n}_{q}^{2} n_{g}^{2}}\right)
\end{aligned}
$$

where $\tilde{n}_{i} \equiv n_{i} / \lambda_{i}$ denote the densities with unit fugacities, $\lambda_{i}=1, \sigma_{3}$ and $\sigma_{2}$ are thermally averaged, velocity weighted cross sections,

$$
\sigma_{3}=\langle\sigma(g g \rightarrow g g g) v\rangle, \quad \sigma_{2}=\langle\sigma(g g \rightarrow q \bar{q}) v\rangle
$$

We have also assumed detailed balance and a baryon symmetric matter, $n_{q}=n_{\bar{q}}$. If we assume that parton scatterings are sufficiently rapid to maintain local thermal equilibrium, and therefore we can neglect effects of viscosity due to elastic [61, 130 and inelastic [123] scatterings, we then have the hydrodynamic equation

$$
\partial_{\mu}\left(\varepsilon u^{\mu}\right)+P \partial_{\mu} u^{\mu}=0
$$

which determines the evolution of the energy density. For a more complete study, one can include the viscosity corrections to the energy-momentum tensor and modify the 
above hydrodynamic equation. This is beyond the scope of this review.

For a time scale $\tau \ll R_{A}$, we can neglect the transverse expansion and consider a purely longitudinal expansion of the parton plasma, which leads to Bjorken's scaling solution 118 of the hydrodynamic equation:

$$
\frac{d \varepsilon}{d \tau}+\frac{\varepsilon+P}{\tau}=0
$$

We further assume an ultrarelativistic equation of state, $\varepsilon=3 P$, with $n_{i}$ and $\varepsilon$ given by Eq. (96). We can then solve the hydrodynamic equation,

$$
\left[\lambda_{g}+\frac{b_{2}}{a_{2}} \lambda_{q}\right]^{3 / 4} T^{3} \tau=\text { const. }
$$

and rewrite the rate equation in terms of the time evolution of the parameters $T(\tau)$, $\lambda_{g}(\tau)$ and $\lambda_{q}(\tau)$

$$
\begin{aligned}
& \frac{\dot{\lambda}_{g}}{\lambda_{g}}+3 \frac{\dot{T}}{T}+\frac{1}{\tau}=R_{3}\left(1-\lambda_{g}\right)-2 R_{2}\left(1-\frac{\lambda_{q}^{2}}{\lambda_{g}^{2}}\right) \\
& \frac{\dot{\lambda}_{q}}{\lambda_{q}}+3 \frac{\dot{T}}{T}+\frac{1}{\tau}=R_{2} \frac{a_{1}}{b_{1}}\left(\frac{\lambda_{g}}{\lambda_{q}}-\frac{\lambda_{q}}{\lambda_{g}}\right),
\end{aligned}
$$

where the density weighted reaction rates $R_{3}$ and $R_{2}$ are defined as

$$
R_{3}=\frac{1}{2} \sigma_{3} n_{g}, \quad R_{2}=\frac{1}{2} \sigma_{2} n_{g}
$$

Notice that for a fully equilibrated system $\left(\lambda_{g}=\lambda_{q}=1\right)$, Eq. (104) corresponds to the Bjorken solution, $T(\tau) / T_{0}=\left(\tau_{0} / \tau\right)^{1 / 3}$.

\subsection{Parton Equilibration Rates}

Often referred to as Landau-Pomeranchuk-Migdal (LPM) effect, the interference in radiation induced by multiple scatterings was first investigated in QED for induced photon radiation off a charged particle propagating through a dense medium [29, 30]. It suppresses those soft photons whose formation time is much larger than the mean free path of the fast particle inside the medium. In QCD, however, the interference pattern is different from the QED case, since multiple scatterings through gluon exchanges will force a quark to change its color along its path. In recent studies [27, 31, 32, pQCD analyses indeed indicated that the LPM effect suppresses soft gluon radiation by multiple scatterings. Due to color exchanges, the interference has some non-Abelian behavior which actually depends on the color representation of the 
jet parton. The same mechanism also causes the color conductivity in a quark-gluon plasma to be small [131, 132.

To account for the LPM effect in the calculation of the reaction rate $R_{3}$ for $g g \rightarrow g g g$, we will use the triple differential cross section from Refs. [27, 31] and apply the LPM suppression to the radiation of gluons whose effective formation time $\tau_{\mathrm{QCD}}$ are much longer than the mean free path $\lambda_{f}$ of multiple scatterings. At the same time, the LPM effect also regularizes the infrared divergency associated with QCD radiation amplitude. However, $\sigma_{3}$ still contains infrared singularities in the gluon propagators associated with QCD scatterings. For an equilibrium system one can in principle apply the resummation technique developed by Braaten and Pisarski [133] to regularize the electric part of the propagators, though the magnetic sector still has to be regulated by an unknown magnetic screening mass which can only be calculated nonperturbatively [134 up to now. Since we are dealing with a nonequilibrium system, Braaten and Pisarski's resummation is not well defined. As an approximation, we will use 135 the Debye screening mass,

$$
\mu_{D}^{2}=\frac{6 g^{2}}{\pi^{2}} \int_{0}^{\infty} k f(k) d k=4 \pi \alpha_{s} T^{2} \lambda_{g}
$$

to regularize all singularities in both the scattering cross sections and the radiation amplitude.

To further simplify the calculation we approximate the LPM suppression factor [31] by a $\theta$-function, $\theta\left(\lambda_{f}-\tau_{\mathrm{QCD}}\right)$. The modified differential cross section for $g g \rightarrow g g g$ is then

$$
\frac{d \sigma_{3}}{d q_{\perp}^{2} d y d^{2} k_{\perp}}=\frac{d \sigma_{\mathrm{el}}^{g g}}{d q_{\perp}^{2}} \frac{d n_{g}}{d y d^{2} k_{\perp}} \theta\left(\lambda_{f}-\tau_{\mathrm{QCD}}\right) \theta\left(\sqrt{s}-k_{\perp} \cosh y\right),
$$

where

$$
\tau_{\mathrm{QCD}}=\frac{C_{A}}{2 C_{2}} \frac{2 \cosh y}{k_{\perp}}
$$

is the effective formation time [27, 31]. The second step-function accounts for energy conservation, and $s=18 T^{2}$ is the average squared center-of-mass energy of the two gluons in the thermal gas. The regularized gluon density distribution induced by a single scattering is,

$$
\frac{d n_{g}}{d y d^{2} k_{\perp}}=\frac{C_{A} \alpha_{s}}{\pi^{2}} \frac{q_{\perp}^{2}}{k_{\perp}^{2}\left[\left(\mathbf{k}_{\perp}-\mathbf{q}_{\perp}\right)^{2}+\mu_{D}^{2}\right]} .
$$

Similarly, the regularized small angle $g g$ scattering cross section is

$$
\frac{d \sigma_{\mathrm{el}}^{g g}}{d q_{\perp}^{2}}=\frac{9}{4} \frac{2 \pi \alpha_{s}^{2}}{\left(q_{\perp}^{2}+\mu_{D}^{2}\right)^{2}}
$$


The mean free path for elastic scatterings is then

$$
\lambda_{f}^{-1} \equiv n_{g} \int_{0}^{s / 4} d q_{\perp}^{2} \frac{d \sigma_{\mathrm{el}}^{g g}}{d q_{\perp}^{2}}=\frac{9}{8} a_{1} \alpha_{s} T \frac{1}{1+8 \pi \alpha_{s} \lambda_{g} / 9},
$$

which depends very weakly on the gluon fugacity $\lambda_{g}$, where again $a_{1}=16 \zeta(3) / \pi^{2} \approx$ 1.95. Using

$$
\int_{0}^{2 \pi} d \phi \frac{1}{\left(\mathbf{k}_{\perp}-\mathbf{q}_{\perp}\right)^{2}+\mu_{D}^{2}}=\frac{2 \pi}{\sqrt{\left(k_{\perp}^{2}+q_{\perp}^{2}+\mu_{D}^{2}\right)^{2}-4 q_{\perp}^{2} k_{\perp}^{2}}}
$$

we can complete part of the integrations and have

$$
R_{3} / T=\frac{32}{3 a_{1}} \alpha_{s} \lambda_{g}\left(1+8 \pi \alpha_{s} \lambda_{g} / 9\right)^{2} \mathcal{I}\left(\lambda_{g}\right)
$$

Here, $\mathcal{I}\left(\lambda_{g}\right)$ is a function of $\lambda_{g}$ :

$$
\begin{array}{rl}
\mathcal{I}\left(\lambda_{g}\right)=\int_{1}^{\sqrt{s} \lambda_{f}} d x \int_{0}^{s / 4 \mu_{D}^{2}} & d z \frac{z}{(1+z)^{2}}\left\{\frac{\cosh ^{-1}(\sqrt{x})}{x \sqrt{\left[x+(1+z) x_{D}\right]^{2}-4 x z x_{D}}}\right. \\
& \left.+\frac{1}{s \lambda_{f}^{2}} \frac{\cosh ^{-1}(\sqrt{x})}{\sqrt{\left[1+x(1+z) y_{D}\right]^{2}-4 x z y_{D}}}\right\},
\end{array}
$$

where $x_{D}=\mu_{D}^{2} \lambda_{f}^{2}$ and $y_{D}=\mu_{D}^{2} / s$. We can evaluate the integration numerically and find out the dependence of $R_{3} / T$ on the gluon fugacity $\lambda_{g}$. In Fig. 24, $R_{3} / T$ is plotted versus $\lambda_{g}$ for a coupling constant $\alpha_{s}=0.3$. The gluon production rate increases with $\lambda_{g}$ and then saturates when the system is in equilibrium.

The calculation of the quark equilibration rate $R_{2}$ for $g g \rightarrow q \bar{q}$ is more straightforward. But one also has to use effective quark and gluon propagators to regularize the singularities for massless quark production [136]. We can also estimate the quark equilibration rate by using the effective thermal quark mass [137,

$$
M_{q}^{2}=\left(\lambda_{g}+\frac{1}{2} \lambda_{q}\right) \frac{4 \pi}{9} \alpha_{s} T^{2}
$$

as a cutoff in the divergent integral over momentum transfer. After averaging over the thermal gluon distribution, we have [119

$$
R_{2}=\frac{1}{2} \sigma_{2} n_{g} \approx 0.24 N_{f} \alpha_{s}^{2} \lambda_{g} T \ln \left(5.5 / \lambda_{g}\right) .
$$




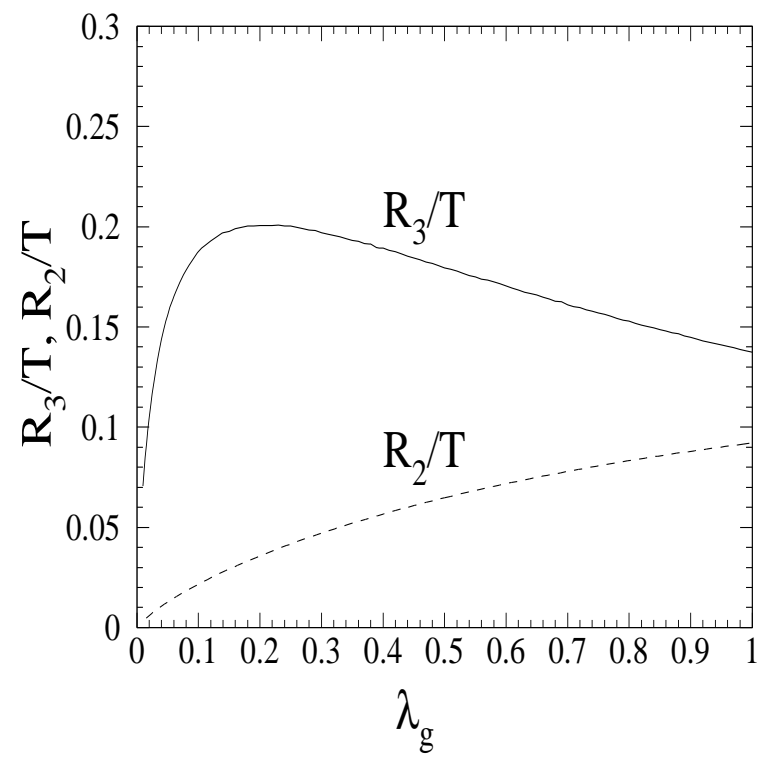

Figure 24: The scaled gluon production rate $R_{3} / T$ (solid line) for $g g \rightarrow g g g$ and the quark production rate $R_{2} / T$ (dashed line) for $g g \rightarrow q \bar{q}$ are shown as function of the gluon fugacity $\lambda_{g}$ for $\alpha_{s}=0.3$.

The dashed line in Fig. 24 shows the normalized rate $R_{2} / T$ for $N_{f}=2.5$, which takes into account the reduced phase space of strange quarks at moderate temperatures, as a function of the gluon fugacity.

\subsection{Evolution of the Parton Plasma}

With the parton equilibration rates which in turn depend on the parton fugacity, we can solve the master equations self-consistently and obtain the time evolution of the temperature and the fugacities. Shown in Figs. 25 and 26 are the time dependence of $T, \lambda_{g}$, and $\lambda_{q}$ for the initial conditions listed in Table 1 at RHIC and LHC energies. We find that the parton gas cools considerably faster than predicted by Bjorken's scaling solution $\left(T^{3} \tau=\right.$ const.) shown by the dotted lines, because the production of additional partons during the approach to chemical equilibrium consumes an appreciable amount of energy. The increased cooling, in turn, impedes the chemical equilibration process; this is more apparent at the RHIC than at the LHC energies. Therefore, the parton system can hardly reach its equilibrium state before the effective temperature drops below $T_{c} \approx 200 \mathrm{MeV}$ in the short period of time of $1-2 \mathrm{fm} / c$ at the RHIC energy. At the LHC energy, however, the parton gas, especially the gluon component, becomes very close to equilibrium (in terms of phase space occupation) and the plasma may exist in a deconfined phase for as long as 4-5 fm/c. Another 


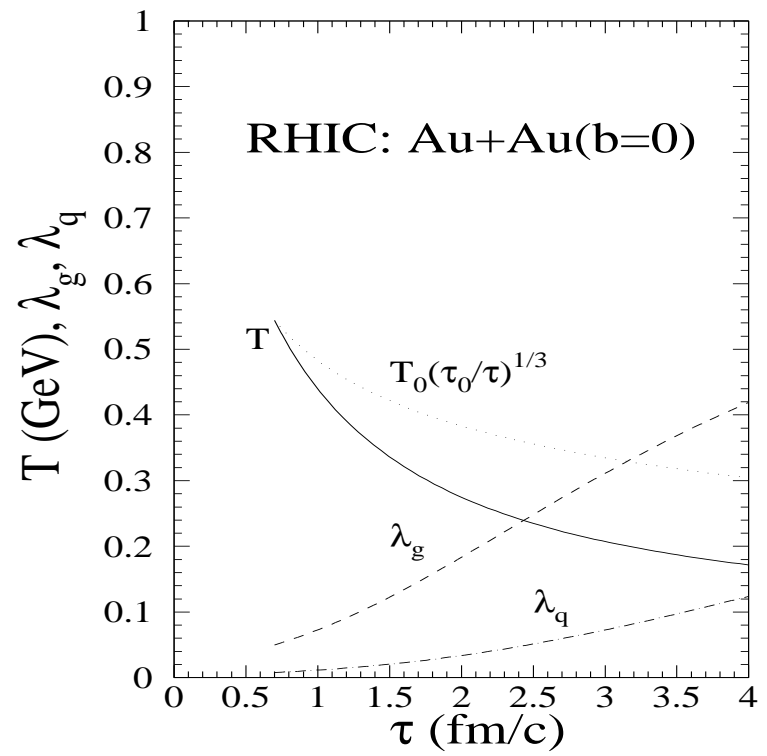

Figure 25: Time evolution of the temperature $T$ and the fugacities $\lambda_{g}$ and $\lambda_{q}$ of gluons and quarks in the parton plasma created in $\mathrm{Au}+\mathrm{Au}$ collisions at the RHIC energy of $\sqrt{s}=200 \mathrm{AGeV}$. The initial values for $T, \lambda_{g}$ and $\lambda_{q}$ are determined from HIJING simulations and are listed in Table 1 .

important observation is that quarks never reach chemical equilibrium at both energies. This is partly due to the small initial quark fugacity and partly due to the small quark equilibration rate.

I should emphasize that the initial conditions used here result from the HIJING model calculation in which only initial direct parton scatterings are taken into account. Due to the fact that HIJING is a QCD motivated phenomenological model, there are some uncertainties related to the initial parton production.

(1) As I have discussed in the second section, the total jet cross section and initial parton production depend on the $p_{T}$ cutoff $p_{0}$. We only included those partons which are produced through a hard or semihard scattering with transverse momentum transfer $p_{T}$ larger than $p_{0}$. Soft partons through soft interactions with $p_{T}<p_{0}$ are locked up in the phenomenological strings which will fragment into hadrons later. How one should include these soft partons in the parton equilibration processes and what value one should choose for $p_{0}$ cannot be answered within the model. The initial parton density is especially sensitive to the value of $p_{0}$.

(2) The dominant initial gluon production is also sensitive to the gluon distribution at small $x$. But so far there is no precise measurement of the gluon distribution in the small $x$ region where most of the minijets originate at RHIC and especially at the LHC energies. The constraints from recent HERA data [58 suggest a more 


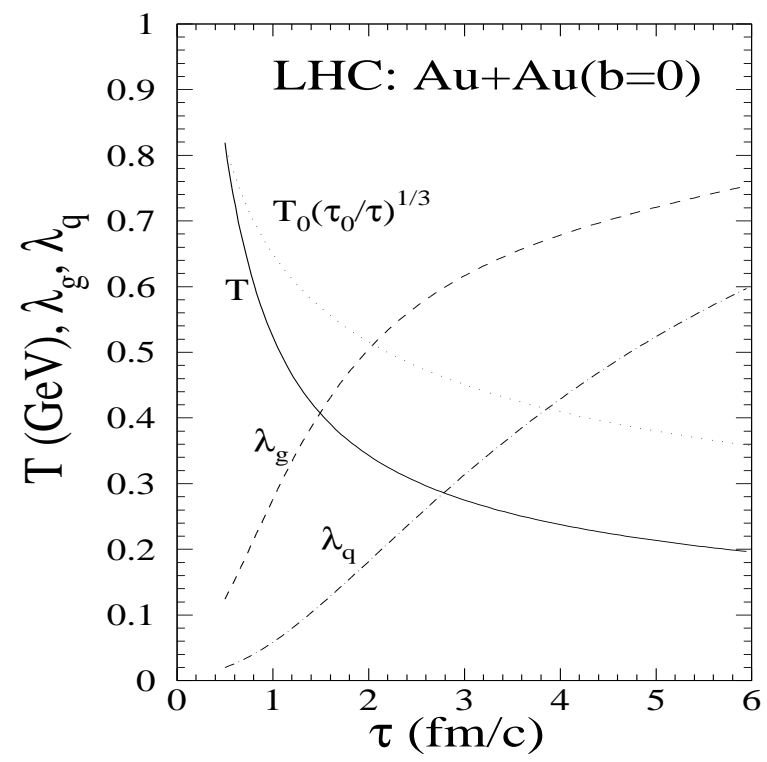

Figure 26: The same as in Fig. 25, except for LHC energy, $\sqrt{s}=5.5 \mathrm{ATeV}$.

singular behavior of parton distributions than the original Duke-Owen parametrization which we have used here. A study in Ref. [59] shows that these more singular gluon distributions can give rise to a much larger amount of produced partons at the LHC energy. Furthermore, we have no handle experimentally so far on the nuclear shadowing of the gluon structure function. In HIJING we have assumed the same shadowing for gluons and quarks, which has reduced the parton production by about half inside a heavy nucleus.

(3) Secondary elastic parton scatterings before $\tau_{\text {iso }}$ have not been considered here, which could speed up the thermalization process. For example, the parton cascade model [20] claims a shorter thermalization time than what we have used here. Changes in the initial thermalization time, therefore, will also lead to uncertainties in the initial energy and parton densities.

We can estimate the effect of the uncertainties in the initial conditions on the parton gas evolution by multiplying the initial energy and parton number densities at RHIC energy by a factor of 4 . This will result in the initial fugacities, $\lambda_{g}^{0}=0.2$ and $\lambda_{q}^{0}=0.024$. With these high initial densities, the parton plasma can evolve into a nearly equilibrated gluon gas, as shown in Fig. 27. The deconfined phase will also last longer, for about $4 \mathrm{fm} / c$. However, the system is still dominated by gluons, and thus has fewer quarks and antiquarks than expected in a fully chemical equilibrated system. If the uncertainties in the initial conditions are caused by the soft parton production from the color mean fields, the initial effective temperature will decrease because soft partons are generally less energetic. Therefore, we can 


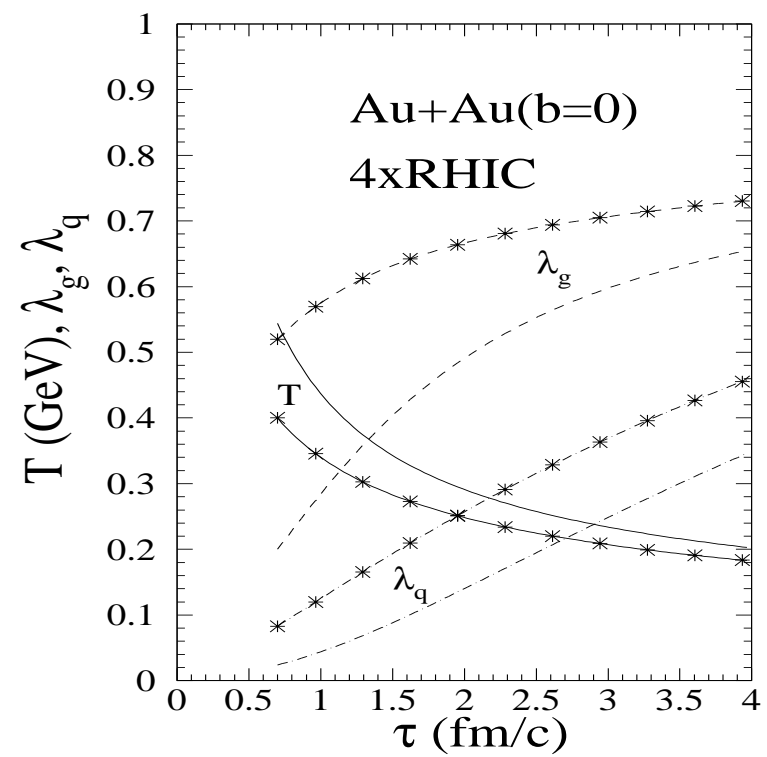

Figure 27: The same as in Fig. 25, except that the initial parton densities are 4 times higher than given in Table 1 with the same (ordinary lines), or reduced initial temperature, $T_{0}=0.4 \mathrm{GeV}$ (lines with stars)

alternatively increase the initial parton density by a factor of 4 and decrease $T_{0}$ to $0.4 \mathrm{GeV}$ at the same time. This leads to higher initial fugacities, $\lambda_{g}^{0}=0.52$ and $\lambda_{q}^{0}=0.083$. As shown in Fig. 27 by the curves with stars, this system evolves faster toward equilibrium, however, with shorter life-time in the deconfined phase due to the reduced initial temperature. We thus can conclude that perturbative parton production and scatterings are very likely to produce a quark-gluon plasma (or gluon plasma to be more accurate) in ultrarelativistic heavy-ion collisions at LHC energy. However, the fate of the quark-gluon plasma at RHIC energy has to be determined by a more careful examination of the uncertainties in the initial conditions.

\subsection{Effects of $g g \rightarrow(n-2) g$ processes}

So far we have only considered $g g \rightarrow g g g$ process in parton equilibration. In fact, higher order processes like $g g \rightarrow(n-2) g$ for $n>5$ should also contribute, where $n$ is the total number of gluons involved in the processes. Even though these processes are higher order in $\alpha_{s}$, the infrared behavior of QCD radiation gives rise to a logarithmic factor $\ln Q^{2}$ after integration which can offset the logarithmically decreasing coupling constant and make the contributions relatively important for all orders in $\alpha_{s}(Q)$. This is especially true for interactions with large momentum transfer $Q$.

The complete amplitude involving $n$-gluon processes in general has contribu- 
tions from different helicity transitions. Parke and Taylor [138 have derived the exact form of the maximum helicity violation amplitude for the $n$-gluon processes,

$$
\left|\mathcal{M}_{n}^{\mathrm{PT}}\right|^{2}=\frac{\left(N_{c} \alpha_{s} / 2 \pi\right)^{n-2}}{N_{c}^{2}-1} \sum_{i>j} s_{i j}^{4} \sum_{\mathrm{P}} \frac{1}{s_{12} s_{23} \cdots s_{n 1}},
$$

where $s_{i j}=\left(p_{i}+p_{j}\right)^{2}$ and the summation $\mathrm{P}$ is over the $(n-2) ! / 2$ non-cyclic permutation of $(1 \cdots n)$. There is no known exact expression for the other chiral amplitudes. Kunszt and Stirling [139 proposed to add a factor to the Parke-Taylor amplitude,

$$
\left|\mathcal{M}_{n}^{\mathrm{KS}}\right|^{2}=C_{\mathrm{KS}}(n)\left|\mathcal{M}_{n}^{\mathrm{PT}}\right|^{2}, \text { with } C_{\mathrm{KS}}(n)=\frac{2^{n}-2(n+1)}{n(n-1)}
$$

to get the total $n$-gluon amplitude. This procedure can be checked to recover the analytic results for $n=4$ and 5 140.

Using the above tree-level $n$-gluon amplitude, Xiong and Shuryak [141] have calculated the $n$-gluon reaction rate,

$$
\begin{aligned}
R_{g g \rightarrow(n-2) g}= & \frac{1}{2 !(n-2) !} \int \frac{d^{3} k_{1}}{(2 \pi)^{3} 2 \omega_{1}} f\left(k_{1}\right) \frac{d^{3} k_{2}}{(2 \pi)^{3} 2 \omega_{2}} f\left(k_{2}\right) \\
& \left|\mathcal{M}_{n}^{\mathrm{KS}}\right|^{2}(2 \pi)^{4} \delta^{4}\left(k_{1}+k_{2}-\sum_{i=3}^{n} k_{i}\right) \prod_{i=3}^{n}\left[1+f\left(k_{i}\right)\right] \frac{d^{3} k_{i}}{(2 \pi)^{3} 2 \omega_{i}}
\end{aligned}
$$

In order to regulate the infrared and collinear divergencies they impose a cut-off,

$$
s_{i j} \geq s_{0}=\eta^{2} T^{2}
$$

in the integration over the phase space. If such a cut-off is provided by the Debye screening mass, then $\eta^{2}=\alpha_{s} / 4 \pi$. Neglecting the temperature dependence of the coupling constant, the rates scale with temperature as

$$
R_{g g \rightarrow(n-2) g}=\alpha_{n}(\eta) T^{4}
$$

where the dimensionless coefficients $\alpha_{n}(\eta)$ 's depend only on the screening parameter $\eta$. Xiong and Shuryak found that for $\eta=1.1, \alpha_{n}(\eta)$ decreases with $n$. They also found that the total contribution to the reaction rate from $n \geq 6$ processes is

$$
\sum_{n=6}^{8} \alpha_{n}(\eta=1.1)=2.56
$$

which is comparable in magnitude to $\alpha_{5}(\eta=1.1)=2.32$ for the $g g \rightarrow g g g$ pro- 
cesses. Therefore, they found that $n$-gluon processes contribute significantly to gluon equilibration.

Two remarks can be made here regarding $n$-gluon processes. As I have discussed earlier, the Landau-Pomeranchuk-Midgal effect is very important to regulate the infrared behavior of parton bremsstrahlung and to suppress soft parton radiation induced by multiple scatterings. It thus reduces the reaction rate of $g g \rightarrow g g g$. One would expect that this effect should also be important in multiple gluon production processes. Unfortunately, the analysis of the LPM effect has not been done so far for multiple particle radiation. In order to do so, one has to understand the space-time structure of the $n$-gluon processes.

Secondly, the Parke-Taylor amplitude is only the tree-level amplitude for $n$ gluon processes. A complete calculation should also include all loop corrections to the same order in $\alpha_{s}$. Take the soft radiation limit for an illustration. In this case the Parke-Taylor amplitude can be factorized. Assume the $n$th soft gluon is radiated with a small angle (corresponding to a transverse momentum $p_{T}$ ) along an energetic one and a fraction $(\mathrm{z})$ of its momentum. The cross section for the $n$-gluon process can then be expressed in terms of that of $(n-1)$-gluon,

$$
\sigma_{n} \approx \int \sigma_{n-1} \frac{\alpha_{s}}{2 \pi} \frac{2 N_{c}}{z} d z \frac{d p_{T}^{2}}{p_{T}^{2}}
$$

With the gluon splitting function, $P_{g \rightarrow g g}(z) \approx 2 N_{c} / z$ for $z \ll 1$, this is much like the formula for successive initial and final state radiation we have encountered earlier in the Monte Carlo simulation [cf. Eq. (77)] except that the Sudakov form factors are missing, which account for the virtual corrections. One can thus include virtual corrections in this soft limit via multiplying the cross section by Sudakov form factors or introducing the ' + function' and $\delta$-function in the splitting function. The virtual corrections for the complete amplitude are very complicated and so far no one has attempted to calculate them. However, once included, they will make the total cross section unitary and likely reduce the gluon multiplication rate calculated from tree level amplitudes.

\section{$5 \quad$ Hard Probes of the Equilibrating Parton Plasma}

So far I have discussed parton production and quark-gluon plasma formation in the framework of a pQCD-based model. In this framework, hard or semihard scatterings among partons play an essential role in liberating partons from the individual confining nucleons and driving the initially produced parton system toward equilibrium. The same kind of hard processes can also be used as direct probes of the early parton 
dynamics and the evolution of the quark-gluon plasma. Among these hard probes, electromagnetic signals, like direct photons and dileptons, are considered more direct since they can escape the dense matter without further interaction. They can thus reveal the dynamics of initial parton production and evolution. Similarly, open charm production, jet quenching due to energy loss and $J / \psi$ suppression can all provide us information about parton scattering and thermalization inside a parton plasma. In particular, preequilibrium $J / \psi$ suppression can reveal evidence of the deconfinement of the parton gas since the $J / \psi$ dissociation cross section with a deconfined parton gas is very different from that with a hadronic gas. For a recent review on signals of quark-gluon plasma, see Ref. 142 by Müller. I will here only discuss hard probes in the framework of an equilibrating parton plasma.

\subsection{Open charm production}

Unlike strange quarks, charm quarks cannot be easily produced during the mixed and hadronic phases of the dense matter since the charm mass is much larger than the corresponding temperature scale. The only period when charm quarks can be easily produced is during the early stage of the parton evolution when the effective temperature is still high. At this stage, the parton gas is still not fully equilibrated yet so that the temperature is only an effective parameter describing the average momentum scale. By measuring this pre-equilibrium charm production, one can thus probe the initial parton density in phase space and shed light on the equilibration time [143].

There are three different contributions to charm production in the history of the evolution of the parton system: (1) initial production similar to minijets; (2) pre-thermal production from secondary parton scatterings during the thermalization, $\tau<\tau_{\text {iso }}$; (3) and thermal production during the parton equilibration, $\tau>\tau_{\text {iso }}$. I will discuss them in the reversed order, starting with the charm production during the final stage of parton equilibration.

\subsubsection{Thermal production during equilibration}

Similar to light quark equilibration, charm quarks are produced through gluon fusion $g g \rightarrow c \bar{c}$ and quark antiquark annihilation $q \bar{q} \rightarrow c \bar{c}$ during the evolution of the parton plasma. However, since the number of charm quarks is very small as compared to gluons and light quarks, we can neglect the back reactions, $c \bar{c} \rightarrow g g, q \bar{q}$ and their effect on the parton evolution. Given the phase-space density of the equilibrating 
partons, $f_{i}(k)$, the differential production rate is then [144],

$$
\begin{aligned}
E \frac{d^{3} A}{d^{3} p}= & \frac{1}{16(2 \pi)^{8}} \int \frac{d^{3} k_{1}}{\omega_{1}} \frac{d^{3} k_{2}}{\omega_{2}} \frac{d^{3} p_{2}}{E_{2}} \delta^{(4)}\left(k_{1}+k_{2}-p-p_{2}\right) \\
& {\left[\frac{1}{2} g_{G}^{2} f_{g}\left(k_{1}\right) f_{g}\left(k_{2}\right)\left|\overline{\mathcal{M}}_{g g \rightarrow c \bar{c}}\right|^{2}+g_{q}^{2} N_{f} f_{q}\left(k_{1}\right) f_{\bar{q}}\left(k_{2}\right)\left|\overline{\mathcal{M}}_{q \bar{q} \rightarrow c \bar{c}}\right|^{2}\right], }
\end{aligned}
$$

where $g_{G}=2\left(N_{c}^{2}-1\right), g_{q}=2 N_{c}$, are the degeneracy factors for gluons and quarks (antiquarks) respectively, $\left|\overline{\mathcal{M}}_{g g \rightarrow c \bar{c}}\right|^{2},\left|\overline{\mathcal{M}}_{q \bar{q} \rightarrow c \bar{c}}\right|^{2}$ are the averaged matrix elements for $g g \rightarrow c \bar{c}$ and $q \bar{q} \rightarrow c \bar{c}$ processes, respectively,

$$
\begin{aligned}
\frac{\left|\overline{\mathcal{M}}_{g g \rightarrow c \bar{c}}\right|^{2}}{\pi^{2} \alpha_{s}^{2}} & =\frac{12}{\hat{s}^{2}}\left(M^{2}-\hat{t}\right)\left(M^{2}-\hat{u}\right)+\frac{8}{3}\left(\frac{M^{2}-\hat{u}}{M^{2}-\hat{t}}+\frac{M^{2}-\hat{t}}{M^{2}-\hat{u}}\right) \\
& -\frac{16 M^{2}}{3}\left[\frac{M^{2}+\hat{t}}{\left(M^{2}-\hat{t}\right)^{2}}+\frac{M^{2}+\hat{u}}{\left(M^{2}-\hat{u}\right)^{2}}\right]-\frac{6}{\hat{s}}\left(2 M^{2}-\hat{t}-\hat{u}\right) \\
& +\frac{6}{\hat{s}} \frac{M^{2}(\hat{t}-\hat{u})^{2}}{\left(M^{2}-\hat{t}\right)\left(M^{2}-\hat{u}\right)}-\frac{2}{3} \frac{M^{2}\left(\hat{s}-4 M^{2}\right)}{\left(M^{2}-\hat{t}\right)\left(M^{2}-\hat{u}\right)}, \\
\frac{\mid \overline{\mathcal{M}}_{\left.q \bar{q} \rightarrow c \bar{c}\right|^{2}}}{\pi^{2} \alpha_{s}^{2}} & =\frac{64}{9 \hat{s}^{2}}\left[\left(M^{2}-\hat{t}\right)^{2}+\left(M^{2}-\hat{u}\right)^{2}+2 M^{2} \hat{s}\right],
\end{aligned}
$$

Due to the small charm density, we can neglect the Pauli blocking of the final charm quarks. For large charm quark mass, $M$, we can approximate the phase-space density $f_{i}(k)$ by a Boltzmann distribution. We further assume that the distributions are boost invariant, i.e.,

$$
f_{i}(k) \approx e^{-k_{T} \cosh (y-\eta)},
$$

where $\eta=0.5 \ln (t+z) /(t-z)$ is the spatial rapidity of a space-time cells at $(t, z)$. Neglecting the transverse expansion, the above assumption implies that the spacetime cell at $(t, z)$ have a flow velocity, $u=(\cosh \eta, \sinh \eta)$. We can now complete the integral over $\eta$ in $\int d^{4} x=\pi R_{A}^{2} \int d \eta d \tau$ and obtain,

$$
\begin{aligned}
\frac{d N_{\mathrm{th}}}{d y d^{2} p_{T}}= & \frac{\pi R_{A}^{2}}{16(2 \pi)^{8}} \int_{\tau_{\text {iso }}}^{\tau_{c}} \tau d \tau \int p_{T 2} d p_{T 2} d \phi_{2} d y_{2} d \phi_{k 1} d y_{k 1} \frac{2 k_{T 1}^{2}}{\hat{s}} 2 K_{0}\left(Q_{T} / T\right) \\
& {\left[\frac{1}{2} g_{G}^{2} \lambda_{g}^{2}\left|\overline{\mathcal{M}}_{g g \rightarrow c \bar{c}}\right|^{2}+g_{q}^{2} N_{f} \lambda_{q}^{2}\left|\overline{\mathcal{M}}_{q \bar{q} \rightarrow c \bar{c}}\right|^{2}\right] }
\end{aligned}
$$

where $K_{0}$ is the modified Bassel function and $\tau_{c}$ is the time when the temperature, $T$, drops below $200 \mathrm{MeV}$. The kinematic variables are chosen such that,

$$
\begin{aligned}
p_{2} & =\left(M_{T 2} \cosh y_{2}, p_{T 2} \cos \phi_{2}, p_{T 2} \sin \phi_{2}, M_{T 2} \sinh y_{2}\right), \quad M_{T 2}=\sqrt{M^{2}+p_{T 2}^{2}} \\
k_{i} & =k_{T i}\left(\cosh y_{k i}, \cos \phi_{k i}, \sin \phi_{k i}, \sinh y_{k i}\right), \quad i=1,2 .
\end{aligned}
$$


The center-of-mass momentum, $Q=\left(Q_{T} \cosh y_{Q}, \mathbf{q}_{\mathbf{T}}, Q_{T} \sinh y_{Q}\right)$, is defined as $Q=$ $p+p 2=k 1+k 2$, and

$$
\begin{aligned}
Q^{2} & =\hat{s}=2\left[M^{2}+M_{T} M_{T 2} \cosh \left(y-y_{2}\right)-p_{T} p_{T 2} \cos \phi_{2}\right] \\
q_{T}^{2} & =p_{T}^{2}+p_{T 2}^{2}+2 p_{T} p_{T 2} \cos \phi_{2}, \\
Q_{T}^{2} & =Q^{2}+q_{T}^{2}=M_{T}^{2}+M_{T 2}^{2}+2 M_{T} M_{T 2} \cosh \left(y-y_{2}\right) .
\end{aligned}
$$

Using these variables and the energy-momentum conservation, we have,

$$
\begin{aligned}
k_{T 1} & =\frac{Q^{2} / 2}{M_{T} \cosh \left(y-y_{k 1}\right)+M_{T 2} \cosh \left(y_{2}-y_{k 1}\right)-q_{T} \cos \phi_{1 q}} \\
\cos \phi_{1 q} & =\left[p_{T} \cos \phi_{k 1}+p_{T 2} \cos \left(\phi_{2}-\phi_{k 1}\right)\right] / q_{T}
\end{aligned}
$$

In the integral over $\tau$, we shall use the time evolution of the temperature, $T(\tau)$, and fugacities, $\lambda_{i}(\tau)$, as given in the previous sections.

\subsubsection{Pre-thermal production}

Before the parton distributions reach local isotropy in momentum space so that the rate equations can be applied to describe the equilibration of the parton system, scatterings among free-streaming partons can also lead to charm production. Since the system during this period consists dominantly of gluons, we shall only consider gluon fusions. To model the phase-space distribution, we take into account the distribution of the initial production points which spread over a region of width

$$
\Delta_{k} \approx \frac{2}{k_{T} \cosh y}
$$

in $z$ coordinate. Assuming free-streaming until $\tau_{\text {iso }}$ and neglecting the expansion in the transverse direction, the correlated phase-space distribution function is given by

$$
f(k, x)=\frac{1}{g_{G} \pi R_{A}^{2}} g\left(k_{T}, y\right) D(\mathbf{x}-\hat{\mathbf{z}} t \tanh y) \theta\left(\tau_{\text {iso }} \cosh y-t\right)
$$

where

$$
D(\mathbf{x})=\frac{e^{-z^{2} / 2 \Delta_{k}^{2}}}{\sqrt{2 \pi} \Delta_{k}} \theta\left(R_{A}-r\right)
$$

is the initial spatial distribution at $t=0$, and

$$
g\left(k_{T}, y\right)=\frac{(2 \pi)^{3}}{k} \frac{d N_{g}}{d y d^{2} k_{T}}=\frac{(2 \pi)^{2}}{k} h\left(k_{T}\right) \frac{1}{2 Y} \theta\left(Y^{2}-y^{2}\right)
$$




\begin{tabular}{ccl}
\hline \hline$\sqrt{s}(\mathrm{TeV})$ & $Y$ & $h\left(k_{T}\right)\left(\mathrm{GeV}^{-} 2\right)$ \\
\hline 0.2 & 2.5 & $1754.4 e^{-k_{T} / 0.9} /\left(k_{T}+0.3\right)$ \\
5.5 & 5.0 & $2.66 \times 10^{7} /\left(k_{T}+2.9\right)^{6.4}$ \\
& & \\
\hline \hline
\end{tabular}

Table 2: Parametrizations of the momentum spectra of the initially produced partons in HIJING calculation. The unit of the transverse momentum $k_{T}$ is $\mathrm{GeV}$

is the parametrization of the parton spectrum given by HIJING simulations. The phase-space distribution is normalized such that $\lim _{t \rightarrow \infty} \int d^{3} x f(k, x) /(2 \pi)^{3}=d^{3} N_{g} / d^{3} k$. The function $h\left(k_{T}\right)$ and the rapidity width $Y$ are given in Table 2 for central $A u+A u$ collisions at RHIC and LHC energies.

Substituting the phase-space distribution, into Eq. (126), and integrating over space and time, we obtain the charm production distribution in the pre-thermal period,

$$
\begin{aligned}
\frac{d N_{\text {pre }}}{d y d^{2} p_{T}}= & \frac{1}{16(2 \pi)^{8} \pi R_{A}^{2}} \int p_{T 2} d p_{T 2} d \phi_{2} d y_{2} d \phi_{k 1} d y_{k 1} \frac{2 k_{T 1}^{2}}{\hat{s}} g\left(k_{T 1}, y_{k 1}\right) g\left(k_{T 2}, y_{k 2}\right) \\
& \frac{1}{2}\left|\overline{\mathcal{M}}_{g g \rightarrow c \bar{c}}\right|^{2} \frac{1}{\sqrt{2 \pi} \Delta_{\text {tot }}} \int_{0}^{t_{f}} d t e^{-t^{2}\left(\tanh y_{k 1}-\tanh y_{k 2}\right) / 2 \Delta_{\text {tot }}^{2}} \\
t_{f} & =\tau_{\text {iso }} \min \left(\cosh y_{k 1}, \cosh y_{k 2}\right), \quad \Delta_{\text {tot }}=\sqrt{\Delta_{k 1}^{2}+\Delta_{k 2}^{2}}
\end{aligned}
$$

where the kinematic variables are similarly defined as in Eq. (130), and in addition,

$$
\begin{aligned}
k_{T 2}^{2} & =Q_{T}^{2}+k_{T 1}^{2}-2 k_{T 1}\left[M_{T} \cosh \left(y-y_{k 1}\right)+M_{T 2} \cosh \left(y_{2}-y_{k 1}\right)\right], \\
\cosh y_{k 2} & =\left[M_{T} \cosh y+M_{T 2} \cosh y_{2}-k_{T 1} \cosh y_{k 1}\right] / k_{T 2} .
\end{aligned}
$$

Note that the correlation between momentum and space-time in the phasespace distribution is very important as discussed by Lin and Gyulassy in Ref. 145, where formation time effects are also included. As we will see, this correlation will reduce the pre-thermal charm production as compared to the result in Ref. [143].

\subsubsection{Initial fusion}

During the initial interaction period, charm quarks are produced together with minijets through gluon fusion and quark anti-quark annihilation. Like gluon and light 


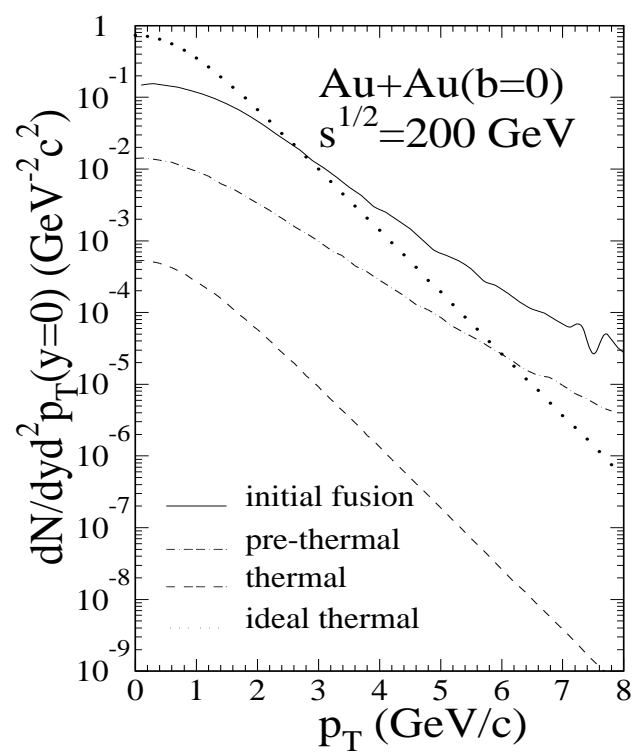

Figure 28: The $p_{T}$ distributions of initial (solid), prethermal (dot-dashed), and thermal (dashed) charm production for central $A u+A u$ collisions at RHIC energy, $\sqrt{s}=200 \mathrm{AGeV}$ with initial conditions given in Tables 1 and 2. The dotted line is the thermal production assuming an initial fully equilibrated QGP at the same temperature.

quark production, charm production through the initial fusion is very sensitive to the parton distributions inside nuclei. In addition, the cross section is also very sensitive to the value of the charm quark mass, $M$. If higher order corrections are taken into account, the production cross section depends also on the choices of the renormalization and factorization scales. Detailed studies of the next-leading-order calculation 146 shows, however, that higher order corrections to the total charm production cross section can be accounted for by a constant $K$ factor of about 2 . This is what we will use next. For consistency we use $M=1.5 \mathrm{GeV}$ for all calculations. Shown in Figs. 28 and 29 as solid lines are the initial charm production given by HIJING calculations at RHIC and LHC energies, with MRSD $-^{\prime}$ [57] parton distributions. The corresponding total integrated cross sections are, $\sigma_{c \bar{c}}=0.16$ (5.75) mb at RHIC (LHC) energy, where nuclear shadowing of the gluon distribution function is also taken into account. In the HIJING calculations, higher order corrections are included via parton cascade in both initial and final state radiations. The resultant distributions in $c \bar{c}$-pair momentum are very close to the explicit higher order calculations [146].

Plotted in Figs. 28 and 29 as dot-dashed and dashed lines are the pre-thermal and thermal production. In the calculation, a factor of 2 is also multiplied to the lowest order matrix elements of charm production. Both contributions are much 


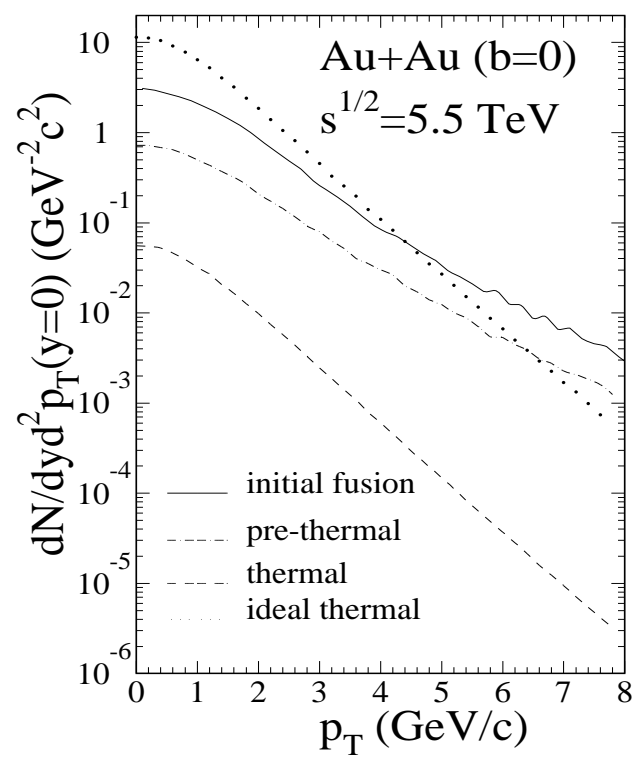

Figure 29: The same as in Fig. 28, except at LHC energy, $\sqrt{s}=5.5 \mathrm{ATeV}$.

smaller than the initial charm production at both energies. The pre-thermal contributions shown are also much smaller than what was found in Ref. [143]. This is because momentum and space-time correlation was not taken into account in Ref. [143] which suppresses the pre-thermal charm production. Similar results are also found in a study by Lin and Gyulassy [145]. However, a fully equilibrated parton plasma $\left(\lambda_{g}=\lambda_{q}=1\right)$ at the same initial temperature would give an enhancement of charm production about 4 times higher than the initial production, shown as dotted lines in Figs. 28 and 29. In this case, the enhancement not only comes from higher parton densities, but also from the much longer life time of the parton plasma (cf. Figs. 25 and 26).

As we have already discussed, the initial conditions in Tables 11 and 2 given by HIJING calculations have many uncertainties. If one increases the initial parton number density at RHIC energy by a factor of 4 with the same initial temperature, charm production from both pre-thermal and thermal sources will increase about a factor of 16 as shown in Fig. 30, leading to a total secondary contribution comparable to the initial charm production. Alternatively, as we have discussed in the Section on parton evolution, we can consider the initial parton densities to be 4 times higher than given in Table 11 at RHIC energy but with lower initial temperature, $T_{0}=0.4 \mathrm{GeV}$. Accordingly, the initial phase-space distribution is also modified to: $h\left(k_{\perp}\right)=9649.2 e^{-k_{\perp} / 0.65} /\left(k_{\perp}+0.3\right)$ from the one in Table 2, which gives 4 times the initial parton density but a smaller average transverse momentum, $\left\langle k_{\perp}\right\rangle=0.85$ $\mathrm{GeV}$. The reduced average transverse momentum corresponds to a lower initial ef- 


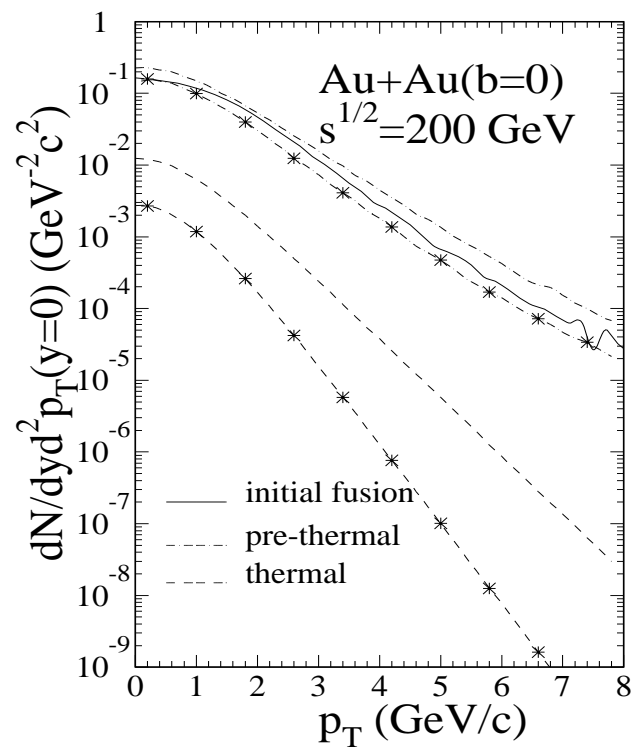

Figure 30: The $p_{\perp}$ distribution of the initial (solid), pre-thermal (dot-dashed) and thermal (dashed) charm production for initial parton densities 4 times higher than the HIJING estimate given in Table \&, with the same (ordinary lines) or reduced initial temperature, $T_{0}=0.4 \mathrm{GeV}$ (lines with stars).

fective temperature. This system with higher initial fugacities evolves faster toward equilibrium but the life-time of the deconfined phase is shorter due to the reduced temperature as we have discussed. The corresponding open charm production is shown in Fig. 30 by the lines with stars. We observe that open charm production from both pre-thermal and thermal contribution is reduced due to the reduction in initial temperature and life-time of the parton plasma, even though the initial fugacities are much higher and the evolution toward equilibrium is faster. Thus, open charm production is much more sensitive to the change in the initial temperature than to the parton fugacities.

We note from Eqs. (130) and (138) that the pre-thermal and thermal charm production depends on the thermalization time $\tau_{\text {iso }}$ and the life time of the parton plasma. Therefore, by measuring the charm enhancement, we can probe both the initial parton phase-space distribution and the thermalization and equilibration time. However, in the most optimistic case, the charm production through thermal and pre-thermal scatterings, cannot be 40 to 50 times higher than the initial direct charm production, as claimed in PCM calculation [126]. A detailed examination exhibited that the copious charm production in Ref. [126] is due to an overestimate of the intrinsic charm production. Though the intrinsic charm production is important in the forward direction at large $x_{f}$ [147], it is strongly suppressed in the mid-rapidity 
region due to interference among pQCD amplitudes to the same order [125].

\subsection{Dilepton and Photon Production}

The above calculation of open charm distribution does not include the effects of secondary scatterings suffered by the produced charm quarks. Though the secondary scatterings do not change the total number of charm quarks, they do modify the final momentum distribution. Therefore, there is no straightforward connection between the shape of charm distribution and the evolution history of the parton plasma. Dileptons and photons, on the other hand, are not subject to final state scatterings due to their week interaction with the medium. Their momentum distribution will remain intact throughout the lifetime of the system, and thus can reveal the evolution history. However, unlike open charm production, low-mass dileptons and small $p_{T}$ photons can be easily produced during the hadronic phase together with contributions from many resonance decays. Recently, it has been argued that the enhancement of low-mass dileptons at CERN SPS energy [148 can be interpreted by the the massshift of the $\rho$ mesons 149. Readers can find detailed discussions about hadronic contributions to dilepton production in Ref. [150]. At very high dilepton mass or large photon $p_{T}$, the production is again dominated by initial hard scatterings. Therefore, to study dilepton and photon production from the parton phase, one has to find a window in phase space in which the thermal partonic contribution is dominant. We will find that this is not necessarily possible for all initial conditions. I will not discuss in detail on how to find this window and other proposed techniques [151] to single out dileptons and photons from thermal parton scatterings. Instead, I will only concentrate on how to calculate dilepton and photon production from an equilibrating parton plasma.

Similar to open charm production, dilepton and photon production from parton scatterings can also be divided into initial, pre-thermal and thermal production. The thermal dilepton production rate can be written as

$$
\frac{d N_{\text {therm }}}{d^{4} x}=\frac{1}{(2 \pi)^{6}} \int \frac{d^{3} k_{1}}{2 \omega_{1}} \frac{d^{3} k_{2}}{2 \omega_{2}} 2 M^{2} \sum_{q} \sigma_{q}(M) f_{q}\left(k_{1}\right) f_{\bar{q}}\left(k_{2}\right),
$$

where $\sigma_{q}(M)=12 e_{q}^{2} 4 \pi \alpha^{2} / 3 M^{2}$ is the cross section of $q \bar{q} \rightarrow \ell^{+} \ell^{-}(M)$ summed over spin and color. Insert $\int d^{4} P \delta^{4}\left(P-k_{1}-k_{2}\right)$ and use $d^{4} P=(1 / 2) d M^{2} d^{2} P_{T} d y$, we can obtain the differential rate as,

$$
\frac{d N_{\text {therm }}}{d^{4} x d M^{2} d y}=\frac{1}{(2 \pi)^{6}} \sum_{q} M^{2} \sigma_{q}(M) \int d^{2} P_{T} d y_{1} d \phi_{1} \frac{k_{T 1}^{2}}{2 M^{2}} f_{q}\left(k_{1}\right) f_{\bar{q}}\left(P-k_{1}\right)
$$


where $k_{T 1}=M^{2} / 2\left[M_{T} \cosh \left(y-y_{1}\right)-P_{T} \cos \phi_{1}\right]$ from the requirement that $\left(P-k_{1}\right)^{2}=$ 0 , and $M_{T}=\sqrt{M^{2}+P_{T}^{2}}$. For large values of $M \gg T$, one can approximate a boost invariant distribution such that

$$
f_{q}\left(k_{1}\right) f_{\bar{q}}\left(P-k_{1}\right) \approx \lambda_{q}^{2} e^{-M_{T} \cosh (y-\eta) / T} .
$$

One can then complete the integration in momentum and space-time, $d^{4} x=\tau d \tau d \eta d^{2} x_{T}$. The total number of thermal dileptons produced during the evolution is, similar to the thermal charm production in Eq. (130),

$$
\frac{d N_{\text {therm }}}{d M^{2} d y}=\frac{\pi R_{A}^{2}}{2(2 \pi)^{4}} \int_{\tau_{\text {iso }}}^{\tau_{c}} \tau d \tau M^{3} T K_{1}(M / T) \sum_{q} \lambda_{q}^{2} \sigma_{q}(M)
$$

This number can be calculated numerically given the time-dependence of the temperature and quark fugacity.

Following the calculation of pre-thermal charm production, we can also compute pre-thermal dilepton yield by substituting $f_{q}(k)$ with a correlated phase-space distribution function similar to the one in Eq. (135) for a free-streaming system. From Eq. (142), we can write down the dilepton yield during the pre-thermal period,

$$
\begin{aligned}
\frac{d N_{\text {pre }}}{d M^{2} d y}= & \frac{M^{2}}{(2 \pi)^{5} \pi R_{A}^{2} g_{q}^{2}} \sum_{q} \sigma_{q}(M) \int P_{T} d P_{T} d y_{1} d \phi_{1} \frac{k_{T 1}^{2}}{2 M^{2}} q\left(k_{T 1}, y_{1}\right) q\left(k_{T 2}, y_{2}\right) \\
& \frac{1}{\sqrt{2 \pi} \Delta_{\text {tot }}} \int_{0}^{t_{f}} d t e^{-t^{2}\left(\tanh y_{1}-\tanh y_{2}\right)^{2} / 2 \Delta_{\text {tot }}^{2}}
\end{aligned}
$$

where the time integral is similarly defines as in Eq. (138), $g_{q}=12$ is the degeneracy of a quark (or anti-quark) with 2 flavors, and

$$
\begin{aligned}
k_{T 2}^{2} & =P_{T}^{2}-2 P_{T} k_{T 1} \cos \phi_{1}+k_{T 1}^{2} \\
\sinh y_{2} & =\left(M_{T} \sinh y-k_{T 1} \sinh y_{1}\right) / k_{T 2} .
\end{aligned}
$$

The quark distribution $q\left(k_{T}, y\right)$ is obtained by multiplying the gluon distribution $g\left(k_{T}, y\right)$ in Eq. (137) by 0.07 which is determined by the the quark-to-gluon ratio in HIJING simulation (see Fig. 23).

To the lowest order, the initial dilepton production for a central $A A$ collision is,

$$
\frac{d N_{\text {initial }}}{d y d M^{2}}=\frac{T_{A A}(0)}{s} \frac{4 \pi \alpha^{2}}{9 M^{2}} \sum_{q} e_{q}^{2}\left[f_{q}\left(x_{1}, M^{2}\right) f_{\bar{q}}\left(x_{2}, M^{2}\right)+f_{q}\left(x_{2}, M^{2}\right) f_{\bar{q}}\left(x_{1}, M^{2}\right)\right],
$$


where $M$ is the invariant mass of the dilepton, $e_{q}$ the fractional charge of each quark $q$, and $x_{1,2}=e^{ \pm y} M / \sqrt{s}$ are the fractional momenta carried by the quark and antiquark. Here we neglect the nuclear effects due to initial multiple interactions. One can also calculate the next-to-leading order (NLO) corrections which generally give a $K$-factor about 1.1-1.4, depending on $M$.

The numerical results of the above dilepton yields during three different stages are plotted in Figs. 31 and 32 for central $A u+A u$ collisions at RHIC and LHC energies. The results of initial dilepton production are taken from a NLO calculation in Ref. [152 with MRS D-' parton distributions. Similar to open charm production, we can see that dilepton production during the equilibration is much smaller than from initial parton scatterings due to small quark number density and the correlation between space and momentum for the produced partons. One also notices that thermal production at low masses becomes larger than the pre-thermal one because of the contribution at later time when the system is approaching chemical equilibrium and the quark fugacity is much larger than its initial value. We can also increase the initial parton density by a factor of 4 as before. The corresponding thermal and pre-thermal dilepton yields will also increase by roughly 16 times, which is, nevertheless, still smaller than the initial production. Only in the extreme limit of complete thermal and chemical equilibrium initial condition can the thermal dilepton yield overcome the shadow of the initial Drell-Yan background in the low and intermediate mass range.

This result is also consistent with earlier estimate by Eskola and Lindfors [153]. My estimate here is smaller than those given by Strickland 154 and Shuryak and Xiong [155] because of the smaller quark fugacity I used here. The effective quark fugacity in Ref. [155] is much larger because it is estimated simply from the parton distribution function in a nucleon without taking into account smaller quark production cross section as compared to the gluon production. Using a parton cascade model, Geiger and Kapusta [156] predicted a total dilepton yield which is 5 times larger than the underlying Drell-Yan even at $M=8 \mathrm{GeV}$ region. A detailed analysis is needed to completely understand their results. Similarly as I discussed in multiple scattering and open charm production, it is very likely that the effect of finite formation time is also at play in this case.

Photon production during parton equilibration can be calculated in the same way as done in Ref. [154]. One problem which is unique in photon production is that the quark screening mass has to be included to regulate the infrared divergency [157]. Such a regularization, however, becomes technically difficult in the calculation of prethermal photon production. However, the final result would be qualitatively similar to dilepton production. 


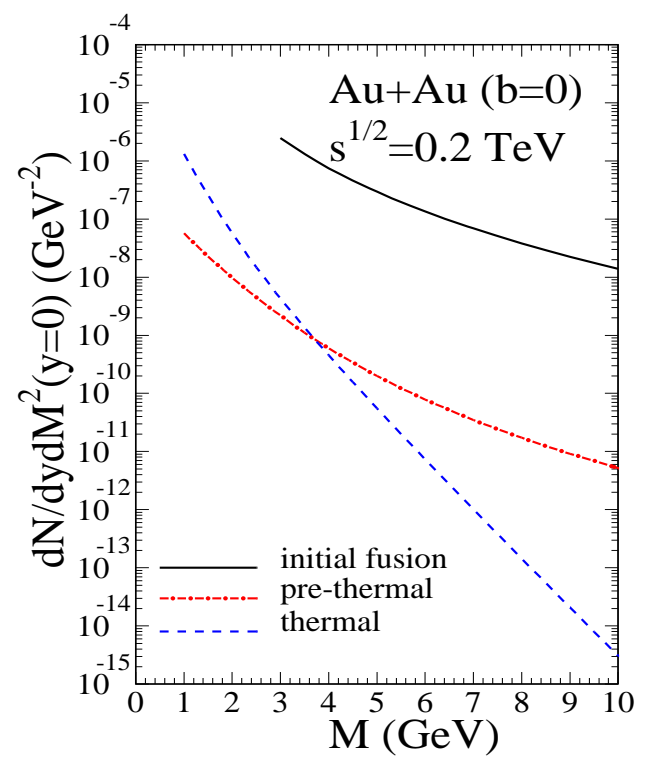

Figure 31: The distributions of initial (solid), pre-thermal (dot-dashed), and thermal (dashed) dilepton production as functions of the invariant mass $M$ for central $A u+A u$ collisions at RHIC energy, $\sqrt{s}=200 \mathrm{AGeV}$ with initial conditions given in Tables [1] and 2 .

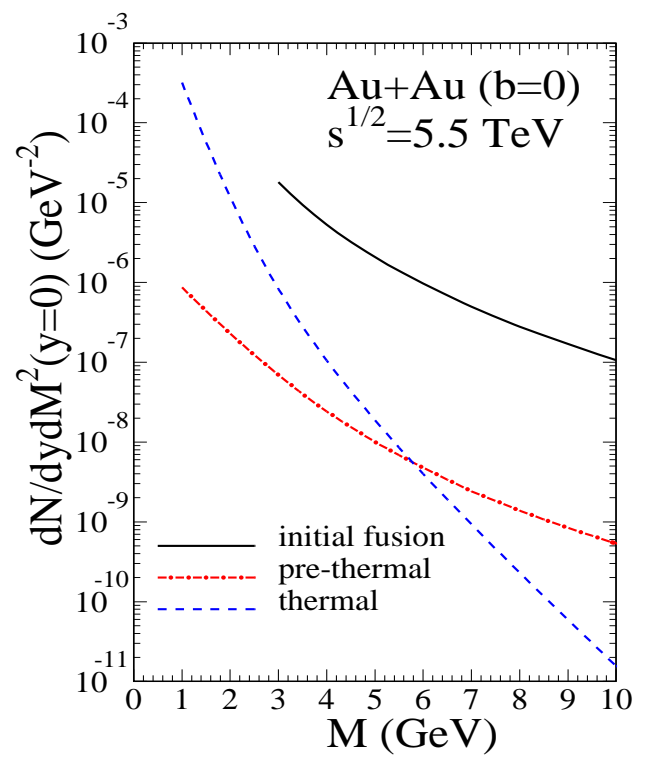

Figure 32: The same as in Fig. 31, except at LHC energy, $\sqrt{s}=5.5 \mathrm{ATeV}$. 
One last note on photon and dilepton production is that background from hadronic decays has to be taken into account in experimental measurements. In particular, dilepton background from charm decays is very important even at relatively large invariant masses 158. One can even use this background, properly identified experimentally, to measure open charm production in $p A$ collisions and the effect of nuclear shadowing [159].

\section{$5.3 J / \psi$ Suppression}

I have demonstrated so far that open charm, dilepton and photon production during the equilibration are very sensitive to the initial parton density and the chemical composition of the initially produced parton system. However, as a careful reader might notice, I have not addressed the question how sensitive these probes are to the color deconfinement of the parton plasma. In principle, charm and dilepton production in a hadronic gas are suppressed by form-factors as compared to a deconfined parton plasma at the same temperature. This is why dilepton production in the hadronic phase contributes mostly to the low mass region while charm production from the hadronic phase can be practically neglected. As we have learned, dileptons and direct photons are only useful when the initial parton density is high enough to produce a signal much larger than the background from the initial parton scatterings. Let us consider another process, $J / \psi$ suppression, which might be more sensitive to the color deconfinement but less dependent on the initial parton density.

Using $J / \psi$ suppression as a probe of the color deconfinement 160 requires that the interactions of $J / \psi$ with hadrons and deconfined partons are different 161. Because of its small size, a heavy quarkonium can probe the short-distance properties of light hadrons. It is thus possible to make a parton-based calculation of the $\mathrm{J} / \psi$ hadron cross section via an operator product expansion method similar to that used in deeply-inelastic lepton-hadron scatterings [161, 162, 163. The resulting $J / \psi$-hadron cross section can be related to the distribution function of gluons inside a hadron. The energy dependence of the cross section near the threshold for the break-up of a $J / \psi$ is determined by the large $x$ behavior of the gluon distribution function, giving rise to a very small break-up cross section at low energies. Only at very high energies, this cross section will reach its asymptotic value of a few mb. Therefore, the dissociation can only occur if the gluon from the light hadron wave function is hard enough in the $J / \psi$ 's rest frame to overcome the binding energy threshold. A hadron gas with temperature below $0.5 \mathrm{GeV}$ cannot provide such energetic gluons to break up the $J / \psi$. Therefore, a slow $J / \psi$ is very unlikely to be absorbed inside a hadron gas of reasonable temperature [161].

On the other hand, a deconfined partonic system contains much harder gluons 


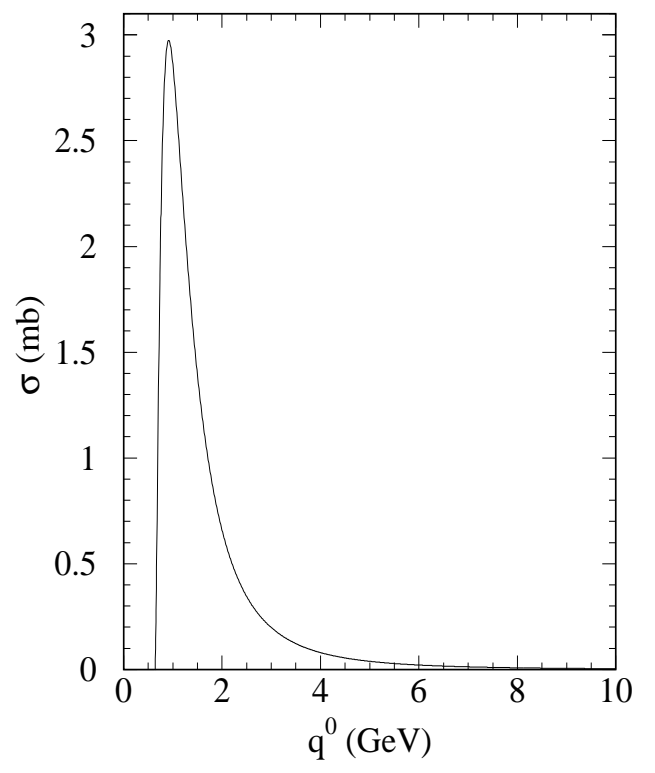

Figure 33: Gluon- $J / \psi$ dissociation cross section as a function of the gluon energy $q^{0}$ in the rest frame of the $J / \psi$.

which can easily break up a $J / \psi$ [161, 164]. The operator product expansion allows one to express the hadron- $J / \psi$ inelastic cross section in terms of the convolution of the gluon- $J / \psi$ dissociation cross section with the gluon distribution inside the hadron [161]. The gluon- $J / \psi$ dissociation cross section is given by [164]

$$
\sigma\left(q^{0}\right)=\frac{2 \pi}{3}\left(\frac{32}{3}\right)^{2} \frac{1}{m_{Q}\left(\epsilon_{0} m_{Q}\right)^{1 / 2}} \frac{\left(q^{0} / \epsilon_{0}-1\right)^{3 / 2}}{\left(q^{0} / \epsilon_{0}\right)^{5}},
$$

with $q_{0}$ the gluon energy in the rest frame of the $J / \psi, m_{Q}$ the heavy quark mass, $\epsilon_{0}=2 M_{D}-M_{J / \psi}$ the binding energy, where $M_{J / \psi}$ and $M_{D}$ are the $J / \psi$ and $D$ meson masses, respectively. As shown in Fig. 33, the inelastic cross section shows a strong peak just above the break-up threshold of the gluon energy, with a maximum value of about $3 \mathrm{mb}$. Low-momentum gluons have neither the resolution to distinguish the heavy constituent quarks nor the energy to excite them to the continuum.

In the pre-equilibrium stage, i.e., before the partons have reached equilibrium, the average parton transverse momentum is sufficiently large to break up a $J / \psi$, provided the partons are deconfined. The dissociation of $J / \psi$ will continue during the whole equilibration process until the effective temperature drops below a certain value or the beginning of hadronization, whichever takes place first. Therefore measurements of $J / \psi$ suppression can probe the deconfinement of the early partonic system and shed light on the subsequent equilibration process, provided that possible nuclear 
effects on the production of $Q \bar{Q}$ pairs and on pre-resonance charmonium states are understood and taken into account.

Let us consider $J / \psi$ suppression in the central rapidity region $\left(y_{J / \psi} \simeq 0\right)$. In this case, the $J / \psi$ will move in the transverse direction with a four-velocity

$$
u=\left(M_{T}, \overrightarrow{P_{T}}, 0\right) / M_{J / \psi},
$$

where $M_{T}=\sqrt{P_{T}^{2}+M_{J / \psi}^{2}}$ is defined as the $J / \psi$ 's transverse mass. A gluon with a four-momentum $k=\left(k^{0}, \vec{k}\right)$ in the rest frame of the parton gas has an energy $q^{0}=k \cdot u$ in the rest frame of the $J / \psi$. Using Eq. (148), one can find out the thermal averaged gluon- $J / \psi$ dissociation cross section [165],

$$
\begin{aligned}
\left\langle v_{\mathrm{rel}} \sigma(k \cdot u)\right\rangle_{k}= & \left(\frac{8}{3}\right)^{3} \frac{\pi}{\zeta(3)} \frac{M_{J / \psi}^{2}}{P_{T} M_{T} T^{3}}\left(\frac{\epsilon_{0}}{m_{Q}}\right)^{3 / 2} \\
& \sum_{n=1}^{\infty} T_{n} \int_{1}^{\infty} d x \frac{(x-1)^{3 / 2}}{x^{4}}\left(e^{-a_{n}^{-} x}-e^{-a_{n}^{+} x}\right),
\end{aligned}
$$

with $T_{n}=T / n$ and

$$
a_{n}^{ \pm}=\frac{\epsilon_{0}}{T_{n}} \frac{M_{T} \pm P_{T}}{M_{J / \psi}} .
$$

Using the thermal cross section just obtained, we can now calculate the survival probability of $J / \psi$ in an equilibrating parton plasma, Again, we will neglect the transverse expansion and consider only longitudinal expansion. A $J / \psi$ produced at point $\vec{r}$ with velocity $\vec{v}$ in the transverse direction will travel a distance

$$
d=-r \cos \phi+\sqrt{R_{A}^{2}-r^{2}\left(1-\cos ^{2} \phi\right)}
$$

in the time interval $t_{\psi}=M_{T} d / P_{T}$ before it escapes from a gluon gas of transverse extension $R_{A}$; here $\cos \phi=\hat{\vec{v}} \cdot \hat{\vec{r}}$. Suppose the system evolves in a deconfined state until the temperature drops below a certain value, which we assume to be $200 \mathrm{MeV}$. The total amount of time the $J / \psi$ remains inside a deconfined parton gas is the smaller one of the two times $t_{\psi}$ and $t_{f}$, the life-time of the parton gas. Assume that the initial production rate of the $J / \psi$ is proportional to the number of binary nucleon-nucleon interactions at impact-parameter $r, N_{A}(r)=A^{2}\left(1-r^{2} / R_{A}^{2}\right) / 2 \pi R_{A}^{2}$. The survival probability of the $J / \psi$ averaged over its initial position and direction in an equilibrating parton gas is

$$
S\left(P_{T}\right)=\frac{\int d^{2} r\left(R_{A}^{2}-r^{2}\right) \exp \left[-\int_{0}^{t_{\min }} d \tau n_{g}(\tau)\left\langle v_{\mathrm{rel}} \sigma(k \cdot u)\right\rangle_{k}\right]}{\int d^{2} r\left(R_{A}^{2}-r^{2}\right)}
$$




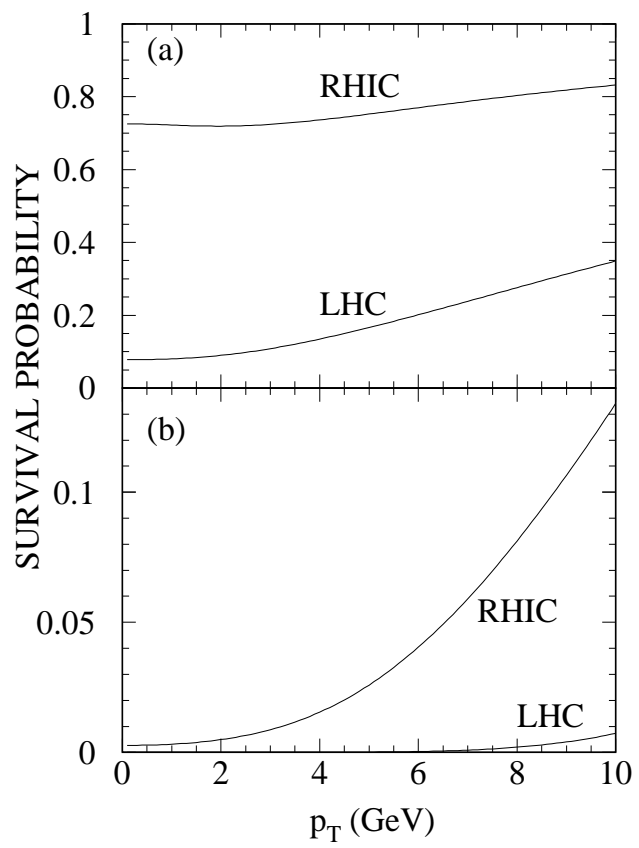

Figure 34: (a) The survival probability of $J / \psi$ in an equilibrating parton plasma at RHIC and LHC energies with initial conditions given by HIJING simulation in [ (b) and for an initially equilibrated plasma at the same temperatures.

where

$$
t_{\min }=\min \left(t_{\psi}, t_{f}\right),
$$

and $n_{g}(\tau)$ is the gluon number density at a given time $\tau$. With the time dependence of the gluon fugacity and temperature we can then evaluate numerically the survival probability of a $J / \psi$ in an equilibrating parton plasma.

Shown in Fig. 34a are the $J / \psi$ survival probabilities in the deconfined and equilibrating parton plasma at RHIC and LHC energies. We find that there is stronger $J / \psi$ suppression at LHC than at RHIC energy, due both to the higher initial parton densities and longer life-time of the parton plasma. The increase of the survival probabilities with $J / \psi$ 's transverse momentum is a consequence of the decrease of the thermal cross section with increasing $P_{T}$ at high temperatures, and the shorter time spent by a higher- $P_{T} J / \psi$ inside the parton plasma, an effect first considered in Ref. [166].

As in open charm and dilepton production, we multiply initial parton number densities by a factor of 4 , thus increasing initial parton fugacities to test the sensitivity of $J / \psi$ suppression to the uncertainties in the initial conditions. The corresponding survival probabilities, shown in Fig. 35 as solid lines, are much lower. If the uncertainties in initial conditions are caused by the soft parton production from the 


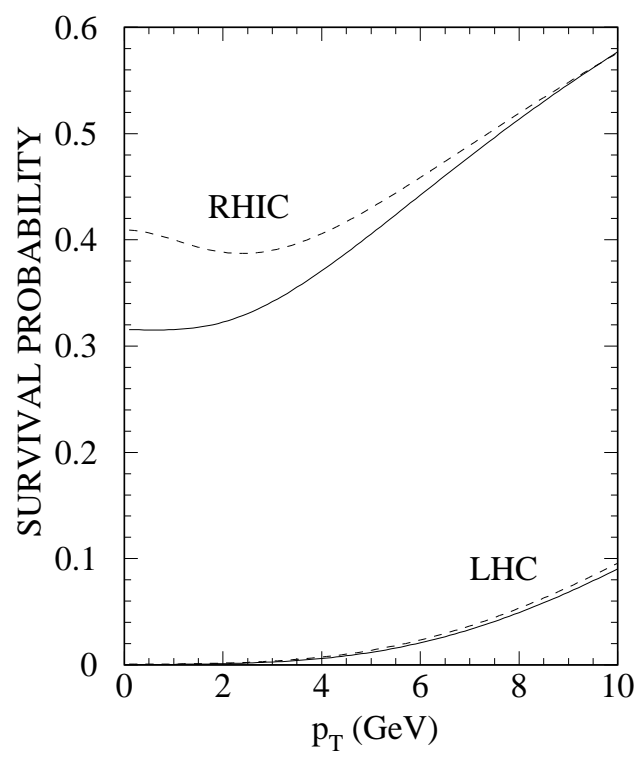

Figure 35: The survival probability of $J / \psi$ in an equilibrating parton plasma with initial parton densities 4 times higher than HIJING estimate given in 1 but with the same (solid line), or reduced initial temperature, $T_{0}=0.4 \mathrm{GeV}$ (dashed line).

color mean fields, the initial effective temperature will decrease. Therefore, we can alternatively increase the initial parton density by a factor of 4 and at the same time decrease $T_{0}$ to 0.4 and $0.72 \mathrm{GeV}$ at RHIC and LHC energies, respectively. This leads also to higher initial fugacities. Comparing the solid and dashed lines shows that the $J / \psi$ suppression is less sensitive to the variation of the initial temperature and fugacities as long as the parton densities are fixed. In the most extreme case of a fully equilibrated parton plasma (unit initial fugacities), $J / \psi$ is much more suppressed, as shown in Fig. 34b, because of both the higher parton density and the longer life time of the system.

At high temperature, the thermal-averaged gluon- $J / \psi$ dissociation cross section decreases monotonically with $P_{T}$ since the averaged collision energy is already above the break-up threshold. One can translate this into a $J / \psi$ survival probability monotonically increasing with $P_{T}$. However, at low temperature blow $300 \mathrm{MeV}$, the $P_{T}$ dependence of the thermally averaged gluon- $J / \psi$ dissociation cross section also displays the threshold peak as in Fig. 33. The peak moves to larger values of $P_{T}$ at a lower temperature [165]. Therefore, due to the large values of the cross section around the threshold peak, the resultant survival probability integrated over the evolution time at low temperatures is quite flat at small $P_{T}$. The final shape of the $P_{T}$ dependence of the survival probability at small $P_{T}$ depends on the entire evolution history of the parton system, especially the relative lengths of time the system spends 
in the high and low temperature stages. The smaller $P_{T}$ dependence at small $P_{T}$ seen in Figs. 34 and 35 is therefore due to the $J / \psi$ dissociation during the late stage of the evolution when the temperature is below $300 \mathrm{MeV}$ [165]. For a parton system with a low initial temperature (below $300 \mathrm{MeV}$ ), the $P_{T}$ dependence of the survival probability should be even flatter. One can therefore use the $P_{T}$ dependence to shed light on the initial temperature and the evolution history of the system.

We have seen that the large average momentum in the hot gluon gas enables gluons to break up the $J / \psi$, while hadron matter at reasonable temperature does not provide sufficiently hard gluons. A substantial $J / \psi$ suppression occurs in such a non-equilibrium partonic medium, though smaller than that in a fully equilibrated parton plasma. I should emphasize that, in addition to the $J / \psi$ dissociation during the equilibration of the parton plasma, there are other possible sources of suppression for the actually observed $J / \psi$ 's, such as nuclear modifications of the $Q \bar{Q}$ production process e.g., through modified gluon distributions in a nucleus [95, 100], multiple scattering accompanied by energy loss [167], or a suppression of the nascent $J / \psi$ before it forms an actual physical resonance [168, 169]. Such effects would cause $J / \psi$ suppression in addition to what we have obtained from the equilibrating parton plasma and modify the transverse momentum dependence of $J / \psi$ suppression [170]. Moreover, there should also be $J / \psi$ pre-thermal suppression. On the other hand, gluon fusion could also result in $J / \psi$ production during the evolution of the parton system, similar to the enhancement of open charm. A consistent study of $J / \psi$ suppression should include the pre-equilibrium production in a form of a master rate equation.

\subsection{Jet Quenching and Monojet Production}

Complementary to $J / \psi$ suppression, a study of high $p_{T}$ jets and their propagation inside the medium can also probe the structure of the dense matter and possibly the phase transition, since these high $p_{T}$ jets are produced on a very short time scale as compared to the soft processes and their production rates and spectrum can be reliably calculated via pQCD. For example, an enhanced acoplanarity of two back-toback jets in nuclear collisions can be used to study multiple scatterings of a parton inside a dense medium [171]. What jets could also probe in high energy heavy ion collisions is the stopping power, $d E / d z$, of the dense matter for high energy quarks and gluons [88, 108, 109. That stopping power in turn is controlled by the color screening mass $\mu_{D}$ in that medium. A possible rapid change of $\mu_{D}$ near the phase transition point could lead to a variation of jet quenching phenomena which may serve as signatures of QGP formation [109]. The energy loss, $d E / d z$, of partons through interaction is also closely related to the thermalization and equilibration of partonic system as we have discussed. 


\subsubsection{Energy loss of a fast parton in QGP}

When an energetic parton propagates through a quark-gluon plasma, it suffers both elastic energy loss, $d E_{\text {el }} / d z$ due to simple scatterings, and radiative energy loss $d E_{\mathrm{rad}} / d z$ due to induced radiations from multiple scatterings. Studies 172, 173 of the elastic energy loss at finite temperatures give a logarithmic energy dependence of $d E_{\text {el }} / d z$. Its value is also quite small, for example, $0.2-0.5 \mathrm{GeV} / \mathrm{fm}$ for a quark jet with $E=30 \mathrm{GeV}$. The dominant source of energy loss is expected to be caused by induced radiations. The estimates of $d E_{\mathrm{rad}} / d z$ in the past vary widely [174, 167, 175, 176] from energy independent to $d E / d z \propto E$. M. Gyulassy and I have analyzed soft gluon bremsstrahlung [27, 31] and found that interference due to multiple scatterings is very important to calculate the radiative energy loss. Similarly to the parton equilibration rate, the LPM effect, i.e., the suppression of soft gluons whose formation times are much larger than the mean free path of parton scatterings, also reduces the radiative energy loss inside a dense QCD medium. Dokshitzer et al. [32] have made a more complete analysis of the QCD radiation amplitude, including also the rescatterings of the radiated gluon. Their results also shown suppression of soft gluon radiation but with a square-root energy-dependence of the energy loss. Their energy loss, $d E / d z$, is also proportional to the distance $z$ the propagating parton has traveled. To demonstrate the LPM effect on energy loss and jet quenching, we will use a simple estimate in this paper.

To estimate the radiative energy loss, we can use the regularized gluon spectrum induced by a single scattering in Eq. (111). We then again take into account the LPM effect in QCD due to color interference by approximating the suppression factor with a step function, $\theta\left(\lambda_{f}-\tau_{\mathrm{QCD}}\right)$. This step function restricts the phase space of the radiation to a region in which the effective formation time $\tau_{\mathrm{QCD}}(k)$ must be smaller than the mean free path $\lambda_{f}$. Radiations not fulfilling this requirement will be suppressed by the destructive interference. The radiative energy loss suffered by the jet parton per collision is then,

$$
\Delta E_{\mathrm{rad}}=\int d^{2} k_{\perp} d y \frac{d n_{g}}{d^{2} k_{\perp} d y} k_{\perp} \cosh y \theta\left(\lambda_{f}-\tau_{\mathrm{QCD}}(k)\right) \theta\left(E-k_{\perp} \cosh y\right),
$$

where $\tau_{\mathrm{QCD}}(k)$ is given by Eq. (110), $E$ is the jet parton energy, and the regularized gluon density distribution, $d n_{g} / d y d^{2} k_{\perp}$, induced by a single scattering is given by Eq. (111). Since the transverse momentum transfer $q_{\perp}$ is the result of elastic scatterings, we have to average any function $f\left(\mathbf{q}_{\perp}\right)$ of $\mathbf{q}_{\perp}$ by the elastic cross section,

$$
\left\langle f\left(\mathbf{q}_{\perp}\right)\right\rangle=\frac{1}{\sigma_{i}} \int_{\mu_{D}^{2}}^{s / 4} d q_{\perp}^{2} \frac{d \sigma_{i}}{d q_{\perp}^{2}} f\left(\mathbf{q}_{\perp}\right),
$$


where $s \approx 6 E T$ is the average $c . m$. energy squared for the scattering of a jet parton with energy $E$ off the thermal partons at temperature $T$. Since we now only consider a fully equilibrated quark-gluon plasma, the Debye screening mass will be given by Eq. (108) with $\lambda_{g}=1$, i.e., $\mu_{D}^{2}=4 \pi \alpha_{s} T^{2}$. For the dominant small angle scattering, the elastic cross sections are,

$$
\frac{d \sigma_{i}}{d q_{\perp}^{2}} \cong C_{\mathrm{el}}^{i} \frac{2 \pi \alpha_{s}^{2}}{q_{\perp}^{4}},
$$

where $C_{\mathrm{el}}^{i}=9 / 4,1,4 / 9$ respectively for $g g, g q$ and $q q$ scatterings. This average can be approximated by replacing $q_{\perp}^{2}$ in the numerator of Eq. (111) with its average value,

$$
\left\langle q_{\perp}^{2}\right\rangle=\mu_{D}^{2} \ln \frac{3 E T}{2 \mu_{D}^{2}} .
$$

In the denominator, we simply replace $\mathbf{q}_{\perp}^{2}$ by $\mu_{D}^{2}$ after the angular integration. The remaining integration in Eq. (155) over the restricted phase space approximately leads to the simple analytic formula,

$$
\begin{gathered}
\Delta E_{\mathrm{rad}} \approx \frac{C_{A} \alpha_{s}}{\pi}\left\langle q_{\perp}^{2}\right\rangle\left(\frac{\lambda_{f}}{2 r_{2}} \mathcal{I}_{1}+\frac{E}{2 \mu_{D}^{2}} \mathcal{I}_{2}\right) \\
\mathcal{I}_{1}=\ln \left[\frac{r_{2} E}{\mu_{D}^{2} \lambda_{f}}+\sqrt{1+\left(\frac{r_{2} E}{\mu_{D}^{2} \lambda_{f}}\right)^{2}}\right]-\ln \left[\left(\frac{r_{2} \sqrt{2}}{\mu_{D} \lambda_{f}}\right)^{2}+\sqrt{1+\left(\frac{r_{2} \sqrt{2}}{\mu_{D} \lambda_{f}}\right)^{4}}\right], \\
\mathcal{I}_{2}=\ln \left[\frac{\mu_{D}^{2} \lambda_{f}}{r_{2} E}+\sqrt{1+\left(\frac{\mu_{D}^{2} \lambda_{f}}{r_{2} E}\right)^{2}}\right]-\ln \left[\frac{2 \mu_{D}^{2}}{E^{2}}+\sqrt{1+\left(\frac{2 \mu_{D}^{2}}{E^{2}}\right)^{2}}\right]
\end{gathered}
$$

In small $k_{\perp}$ regime, the phase space is mainly restricted by a small effective formation time, $\tau_{\mathrm{QCD}}<\lambda_{f}$, which gives the first term proportional to $\lambda_{f}$. For large $k_{\perp}$, the radiation becomes additive in a restricted phase space constrained by energy conservation. That region contributes to the second term which appears to be proportional to the incident energy $E$. However, in the high energy limit the function $\mathcal{I}_{2} \propto 1 / E$ and hence the radiated energy loss grows only as $\log ^{2} E$.

The above derivation assumed that the mean free path is much larger than the interaction range specified by $1 / \mu_{D}$. As we shall discuss below, this is satisfied in a quark gluon plasma at least in the weak coupling limit. Therefore, we can neglect the second term in $\mathcal{I}_{1}$. For a high energy jet parton, $E \gg \mu_{D}$, we can also neglect the second term in $\mathcal{I}_{2}$. The resulting radiative energy loss reduces in that case to the 
simple form,

$$
\frac{d E_{\mathrm{rad}}}{d z}=\frac{\Delta E_{\mathrm{rad}}}{\lambda_{f}} \approx \frac{C_{2} \alpha_{s}}{\pi}\left\langle q_{\perp}^{2}\right\rangle\left[\ln \left(\xi+\sqrt{1+\xi^{2}}\right)+\xi \ln \left(\frac{1}{\xi}+\sqrt{1+\frac{1}{\xi^{2}}}\right)\right],
$$

which depends on a dimensionless variable,

$$
\xi=\frac{r_{2} E}{\mu_{D}^{2} \lambda_{f}}
$$

We see that the radiative energy loss $d E_{\mathrm{rad}} / d z$ thus obtained interpolates between the factorization and Bethe-Heitler limits as a function of the dimensionless variable $\xi$. In the factorization limit, we fix $\mu_{D} \lambda_{f} \gg 1$ and let $E \rightarrow \infty$, so that $\xi \gg 1$. In this case, we can neglect the second term in Eq. (162) and have,

$$
\frac{d E_{\mathrm{rad}}}{d z} \approx \frac{C_{2} \alpha_{s}}{\pi}\left\langle q_{\perp}^{2}\right\rangle \ln \left(\frac{2 r_{2} E}{\mu_{D}^{2} \lambda_{f}}\right) ; \xi \gg 1
$$

Thus, the radiative energy loss in the factorization limit has only a logarithmic energy dependence (in addition to the energy dependence of $\left\langle q_{\perp}^{2}\right\rangle$ ). Due to the non-Abelian nature of the color interference, the resultant energy loss for a gluon $\left(C_{2}=C_{A}\right)$ is $9 / 4$ times larger than that for a quark $\left(C_{2}=C_{F}\right)$. In the other extreme limit, we fix $E$ and let $\mu_{D} \lambda_{f} \rightarrow \infty$, so that $\xi \ll 1$. In this case, the mean-free-path exceeds the effective formation time. The radiation from each scattering adds up. We then recover the linear dependence of the energy loss $d E_{\mathrm{rad}} / d z$ on the incident energy $E$ (modulo logarithms),

$$
\frac{d E_{\mathrm{rad}}}{d z} \approx \frac{C_{A} \alpha_{s}}{2 \pi \lambda_{f}} \frac{\left\langle q_{\perp}^{2}\right\rangle}{\mu_{D}^{2}} E \ln \left(\frac{2 \mu_{D}^{2} \lambda_{f}}{r_{2} E}\right) ; \quad \xi \ll 1,
$$

as in the Bethe-Heitler formula. In both cases, the radiative energy loss is proportional to the average of the transverse momentum transfer, $\left\langle q_{\perp}^{2}\right\rangle$, which is controlled by the color screening mass as in Eq. (158).

To see more clearly how the factorization limit is approached, we now estimate $\xi$ for a parton propagating inside a high temperature quark-gluon plasma. From Eq. (157) and the quark and gluon densities in an ideal system at a temperature $T$, the mean free path (for 3 quark flavors) is

$$
1 / \lambda_{f}^{(q)}=\sigma_{q q} \rho_{q}+\sigma_{q g} \rho_{g} \approx \frac{2 \pi \alpha_{s}^{2}}{\mu_{D}^{2}} 4 \times 7 \zeta(3) \frac{T^{3}}{\pi^{2}}
$$




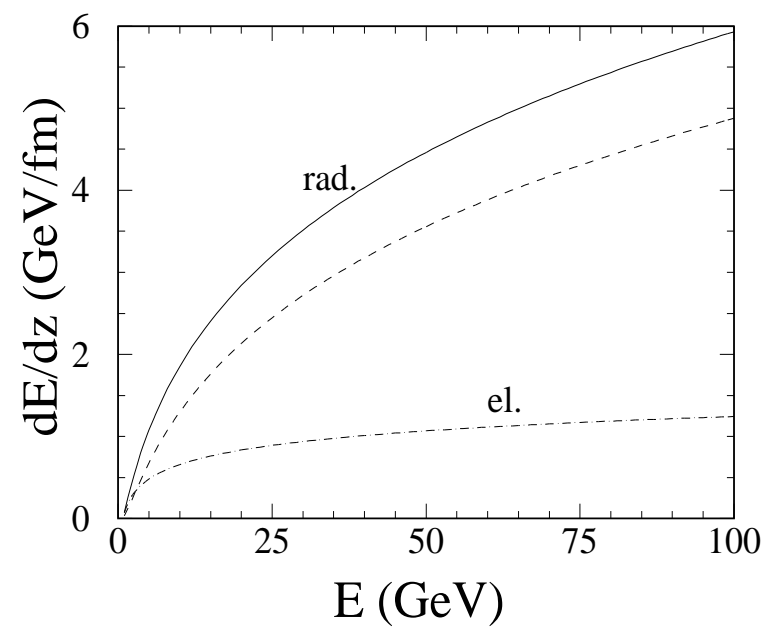

Figure 36: The energy dependence of energy loss, $d E / d z$, of a quark with energy $E$ inside a quark-gluon plasma at temperature $T=300 \mathrm{MeV}$. A weak coupling $\alpha_{s}=0.3$ is used. The solid line is the full expression and the dashed line is the factorization limit of the radiative energy loss. The dot-dashed line is the elastic energy loss.

$$
1 / \lambda_{f}^{(g)}=\sigma_{q g} \rho_{q}+\sigma_{g g} \rho_{g} \approx \frac{2 \pi \alpha_{s}^{2}}{\mu_{D}^{2}} 9 \times 7 \zeta(3) \frac{T^{3}}{\pi^{2}},
$$

where $\zeta(3) \approx 1.2$.

Using Eqs. (166) and (167) and the perturbative color electric screening mass, $\mu_{D}^{2}=4 \pi \alpha_{s} T^{2}$, we see that $\xi$ appearing in the logarithms has a common energy and temperature dependence for both quarks and gluons,

$$
\xi=\frac{r_{2} E}{\lambda_{f} \mu_{D}^{2}}=\frac{63 \zeta(3)}{16 \pi^{3}} \frac{E}{T} \approx \frac{9}{2 \pi^{3}} \frac{E}{T}, \text { for both } q \text { and } g .
$$

With the above expression for $\xi$, we plot the radiative energy loss in Fig. 36 as a function of the jet energy inside a plasma at temperature $T=300 \mathrm{MeV}$ with $\alpha_{s}=0.3$. The solid line is the full expression in Eq. (162) while the dashed line is the factorization limit corresponding to the first term in Eq. (162). We see that Eq. (164) approximates Eq. (162) quite well in this parameter range. The energy dependence of the radiative energy loss is due to the double logarithmic function in the formula one of which comes from the energy dependence of the average transverse momentum $\left\langle q_{\perp}^{2}\right\rangle$ in Eq. (158).

The energy loss of a quark in dense matter due to elastic scattering was first estimated by Bjorken [172] and later was studied in detail [173 in terms of finite temperature QCD. For our purpose, a simple estimate taking into account both the thermal average and color screening will suffice. In terms of elastic cross sections and 
the density distributions for quarks and gluons in a plasma, we have,

$$
\frac{d E_{\mathrm{el}}}{d z}=\int_{\mu_{D}^{2}}^{s / 4} d q_{\perp}^{2} \frac{d \sigma_{i}}{d q_{\perp}^{2}} \rho_{i} \nu=\left\langle q_{\perp}^{2}\right\rangle \sigma_{i}\left\langle\frac{\rho_{i}}{2 \omega}\right\rangle,
$$

where $\nu \approx q_{\perp}^{2} / 2 \omega$ is the energy transfer of the jet parton to a thermal parton with energy $\omega$ during an elastic scattering, $\left\langle q_{\perp}^{2}\right\rangle$ is the average transverse momentum transfer given by Eq. (158). Similar to Eqs. (166) and (167), we have,

$$
\begin{gathered}
\sigma_{q q}\left\langle\frac{\rho_{q}}{2 \omega}\right\rangle+\sigma_{q g}\left\langle\frac{\rho_{g}}{2 \omega}\right\rangle=\frac{2 \pi \alpha_{s}^{2}}{\mu_{D}^{2}} T^{2}, \\
\sigma_{g q}\left\langle\frac{\rho_{q}}{2 \omega}\right\rangle+\sigma_{g g}\left\langle\frac{\rho_{g}}{2 \omega}\right\rangle=\frac{9}{4} \frac{2 \pi \alpha_{s}^{2}}{\mu_{D}^{2}} T^{2} .
\end{gathered}
$$

The elastic energy loss of a fast parton inside a quark gluon plasma at a temperature $T$ is then given by,

$$
\frac{d E_{\mathrm{el}}}{d z}=C_{2} \frac{3 \pi \alpha_{s}^{2}}{2 \mu_{D}^{2}} T^{2}\left\langle q_{\perp}^{2}\right\rangle .
$$

For comparison, we plot this elastic energy loss in Fig. 36. In general, it is much smaller than the radiative energy loss and has a weaker energy dependence (single logarithmic).

Using Eqs. (158), (164) and (168), the total energy loss can be expressed as,

$$
\frac{d E}{d z}=\frac{d E_{\mathrm{el}}}{d z}+\frac{d E_{\mathrm{rad}}}{d z} \approx \frac{C_{2} \alpha_{s}}{\pi} \mu_{D}^{2} \ln \frac{3 E T}{2 \mu_{D}^{2}}\left(\ln \frac{9 E}{\pi^{3} T}+\frac{3 \pi^{2} \alpha_{s}}{2 \mu_{D}^{2}} T^{2}\right) .
$$

It is interesting to note that both the elastic and radiative energy loss have the same color coefficient $C_{2}$. For high energy partons, the radiative energy loss dominates over the elastic one. For $E=30 \mathrm{GeV}, T=300 \mathrm{MeV}$, and $\alpha_{s} \approx 0.3$, the total energy loss for a propagating quark is $d E / d z \approx 3.6 \mathrm{GeV} / \mathrm{fm}$. Only about $25 \%$ of this amount comes from elastic energy loss.

Comparing to the phenomenological string tension $\kappa \sim 1-2 \mathrm{GeV} / \mathrm{fm}$, which can be considered as the $d E / d z$ of quarks in the nonperturbative QCD vacuum 178, one cannot really distinguish jet quenching caused by energy loss in a quark gluon plasma from that in a hadronic matter. However, since the energy loss is very sensitive to the infrared cutoff scale $\mu_{D}(T)$, the study of jet quenching may be used as a signature of a quark gluon plasma if the $\mu_{D}(T)$ dependence of $d E / d z$ can still be given by Eq. (173) and if there is a significant variation in $\mu_{D}(T)$ near the phase transition temperature $T_{c}$. Up to now, little is known about $\mu_{D}(T)$ for physical QCD. However, lattice simulations [177] of a pure gauge QCD indicate a rapid decrease 


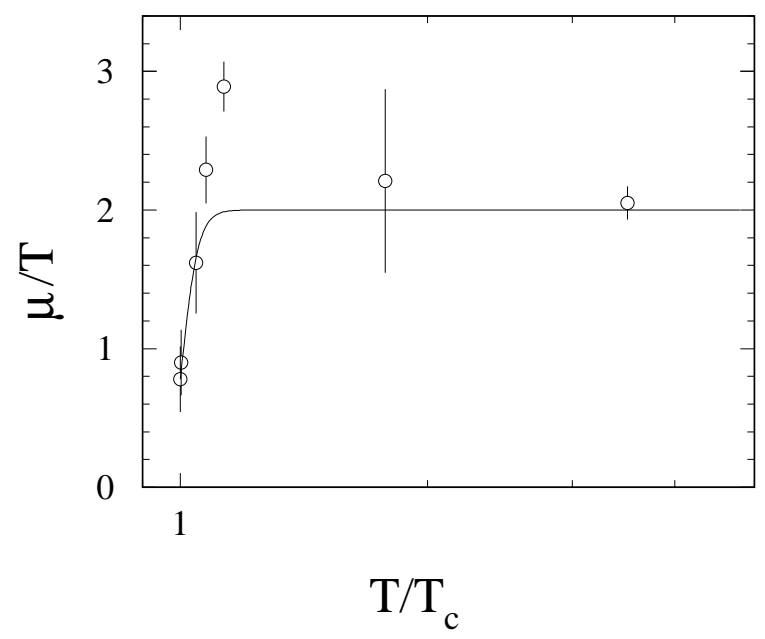

Figure 37: Lattice result of the Debye screen mass [177], $\mu_{D}$, as a function of temperature. The curve is a parametrization used in the following calculation.

of $\mu_{D}(T)$ near $T_{c}$ as shown in Fig. 37, where the weak coupling limit, $\mu(T)=g T$, is indeed recovered at high temperatures. Since a sharp reduction of $\mu_{D}(T)$ would effectively suppress the energy loss according to our formula in Eq. (173), a long duration of the mixed phase in a near-first order phase transition could lead to a decrease of jet quenching.

In the following, we will use a parametrization of the lattice result as a phenomenological guide to describe the temperature dependence of the color screening mass $\mu_{D}(T)$ both near and above $T_{c}$. This will give us the temperature dependence of the energy loss according to Eq. (173). The coupling constant which corresponds to $\mu_{D}(T)=g T$ in Fig. 37 at high temperatures is $\alpha_{s} \approx 0.3$. We shall freeze the coupling constant to this value at all temperatures in calculating $d E / d z$ in the QGP phase. In hadronic phase at $T=0$, we assume a constant energy loss given by the string tension, $\kappa \sim 1 \mathrm{GeV} / \mathrm{fm}$, which is consistent with the deep inelastic lepton-nucleus scattering data [179]. To extrapolate from $T=0$ to $T_{c}$, one may choose a smooth form, $d E / d z \rightarrow 1 \mathrm{GeV} / \mathrm{fm}$ as $T \rightarrow 0$. This scenario is plotted in Fig. 38 for a quark jet with $E=30 \mathrm{GeV}$ as set 1 , in which there is a dip in $d E / d z$ during the phase transition due to the small value of $\mu_{D}$ at $T_{c}$. We have assumed a QCD phase transition temperature $T_{c}=0.188 \mathrm{GeV}$. Alternatively, one may consider a scenario (set 2 in Fig. 38) in which $d E / d z=$ cont. $=1 \mathrm{GeV} / \mathrm{fm}$ in the hadronic phase, i.e., there is a discontinuity in $d E / d z$ at $T=T_{c}$. For comparison, we also study the case that $d E / d z$ in the $T=0$ limit is $2 \mathrm{GeV} / \mathrm{fm}$ (set 3 ). 


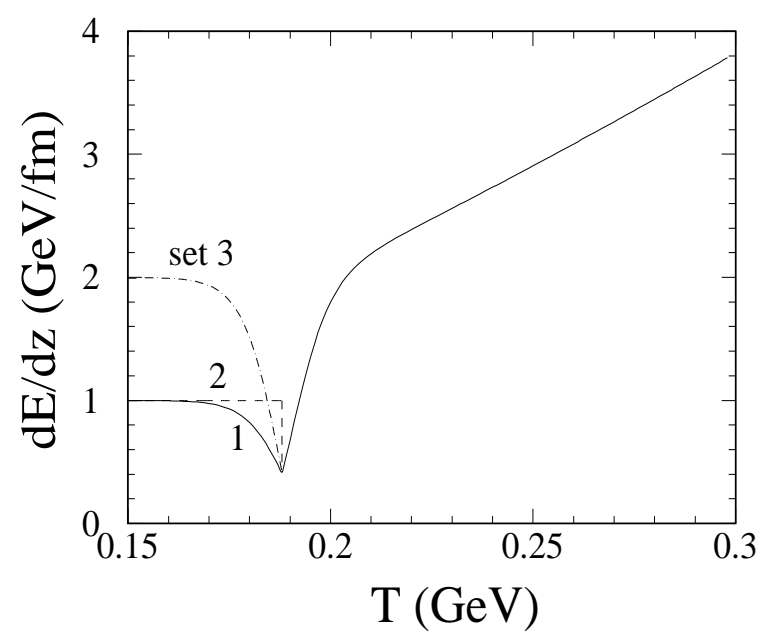

Figure 38: Three scenarios of energy loss as functions of temperature.

\subsubsection{Suppression of dijets}

We now discuss the implications of the temperature dependence of $d E / d z$ on the suppression of di-jets and on the production rate of mono-jets in ultrarelativistic nuclear collisions. Similarly to Eq. (19), the initial rate of back-to-back parton jets of transverse momentum $p_{T}$ and rapidities $y_{1}$ and $y_{2}$ produced in hard scattering processes in central $A A$ collisions is,

$$
\frac{d N_{\text {dijet }}^{(0)}}{d p_{T}^{2} d y_{1} d y_{2}}=\int d^{2} b T_{A}^{2}(b) K \sum_{a, b, c, d} x_{1} f_{a / N}\left(x_{1}, p_{T}^{2}\right) x_{2} f_{b / N}\left(x_{2}, p_{T}^{2}\right) \frac{d \sigma^{a b \rightarrow c d}}{d \hat{t}}(\hat{s}, \hat{t}, \hat{u})
$$

where we have neglected the nuclear shadowing effect for large $p_{T}$ jet production, i.e., $f_{a / A}\left(x, Q^{2}\right) \approx f_{a / N}\left(x, Q^{2}\right) T_{A}(b), T_{A}(b)$ is the nuclear thickness function as defined in Eq. (66). The integration is performed over the plane transverse to the beam direction, $\mathbf{b}$ is the transverse coordinate of the production point in the transverse plane.

In the presence of dense matter, the high energy partons produced by a hard scattering process can interact in the medium and thereby lose energy which will not show up in the final state of the jet but contribute to the soft background. Thus, the number of QCD hard jets to be observed in $A A$ collisions will be reduced as compared to the expression Eq. (174) (in Ref. [108 the term "jet quenching" has been introduced for this effect). Taking into account interactions and energy loss in the medium, the rate of di-jets of rapidities $y_{1}, y_{2}$ and transverse momenta $p_{T 1}, p_{T 2}$ 
reads

$$
\begin{aligned}
\frac{d N_{\text {dijet }}}{d p_{T 1} d p_{T 2} d y_{1} d y_{2}}= & \int d^{2} b \int d p_{T}^{2} T_{A}^{2}(b) K \sum_{a, b, c, d} x_{1} f_{a / N}\left(x_{1}, p_{T}^{2}\right) x_{2} f_{b / N}\left(x_{2}, p_{T}^{2}\right) \\
& \cdot \frac{d \sigma^{a b \rightarrow c d}}{d \hat{t}}(\hat{s}, \hat{t}, \hat{u}) \delta\left(p_{T 1}-p_{T}+\Delta E_{c, 1}\left(\mathbf{b}, \mathbf{p}_{\mathbf{T}}, y_{1}\right)\right) \\
& \cdot \delta\left(p_{T 2}-p_{T}+\Delta E_{d, 2}\left(\mathbf{b}, \mathbf{p}_{\mathbf{T}}, y_{2}\right)\right)
\end{aligned}
$$

The energy losses $\Delta E_{c, 1}$ and $\Delta E_{d, 2}$ depend not only on the momenta and on the production point of the partons $c$ and $d$ but also on the space-time evolution of the dense matter. If we limit ourselves to jets in the central region, $y_{1}=y_{2}=0$, the total energy loss of a massless parton inside the dense matter is

$$
\Delta E=\int_{\tau_{0}}^{\tau_{f}} d \tau \frac{d E}{d z}(\tau)
$$

where $\tau_{0}$ is the formation time of the dense matter and $\tau_{f}$ is the proper time when the parton escapes from the dense medium, $\tau_{f}=\left(R^{2}-b^{2} \sin ^{2} \psi\right)^{1 / 2}-b \cos \psi$, where $\psi$ is the angle between $\mathbf{b}$ and $\mathbf{p}_{\mathbf{T}}$.

To evaluate the integrated energy loss suffered by a fast parton, we now need to know the the time dependence of the temperature. We shall assume a one-dimensional scaling expansion of the system [118], thus the time dependence of the temperature in both phases is given by $T \sim 1 / \tau^{1 / 3}$. To determine the duration of the mixed phase of a first order phase transition in which the temperature is constant, $T_{c}$, we adopt a bag model equation of state (EOS) for the quark-gluon-plasma (QGP) phase:

$$
\varepsilon(T)=\frac{37}{30} \pi^{2} T^{4}+B \quad P(T)=\frac{1}{3}[\varepsilon(T)-4 B]
$$

where $\varepsilon, P$ and $B$ are the energy density, the pressure and the bag constant, respectively. Define the energy densities at the critical temperature in the QGP phase and in the hadronic phase, $\varepsilon_{Q}$ and $\varepsilon_{H}$, respectively. The system then enters the mixed phase at the proper time $\tau_{Q}=\tau_{0} T_{0}^{3} / T_{c}^{3}$, and goes from the mixed phase into the hadronic phase at $\tau_{H}=\left(\varepsilon_{Q}+P_{c}\right) \tau_{Q} /\left(\varepsilon_{H}+P_{c}\right)$, where $P_{c}=\left(\varepsilon_{Q}-4 B\right) / 3$ is the pressure at $T_{c}$. In the following, we shall use $\varepsilon_{Q}=2.5 \mathrm{GeV} / \mathrm{fm}^{3}, \varepsilon_{H}=0.5 \mathrm{GeV} / \mathrm{fm}^{3}$, and $B=0.5 \mathrm{GeV} / \mathrm{fm}^{3}$, which implies a critical temperature $T_{c}=0.188 \mathrm{GeV}$.

In order to achieve maximum sensitivity to the quenching effect it is useful to consider the total transverse energy $E_{\text {tot }}=p_{T 1}+p_{T 2}$ for a pair of back-to-back jets. The quenching ratio is defined as the number of dijets of energy $E_{\text {tot }}$ as measured in the experiment, divided by the corresponding number expected in the absence of jet 


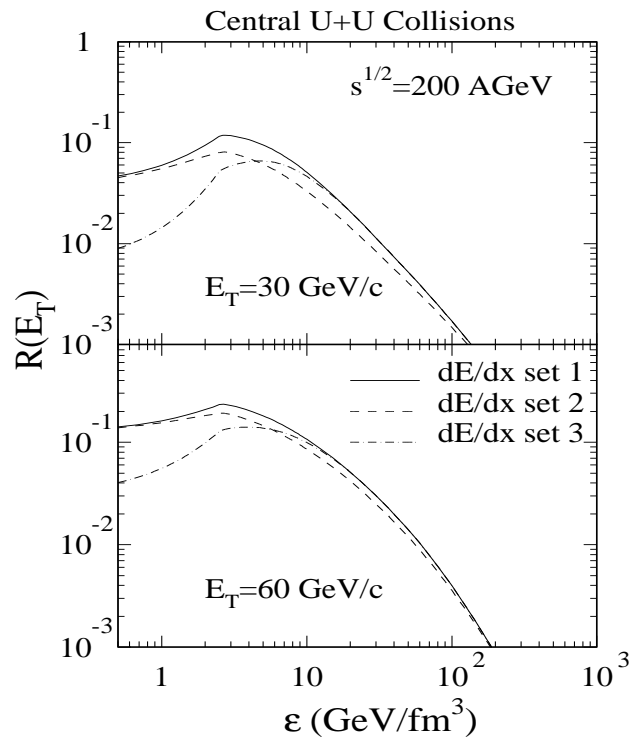

Figure 39: The suppression rate as a function of the initial energy density $\varepsilon_{0}$ for a dijet with the total energy $E_{T}=30,60 \mathrm{GeV}$ in a central $U+U$ collisions at $\sqrt{s}=200$ $\mathrm{GeV}$.

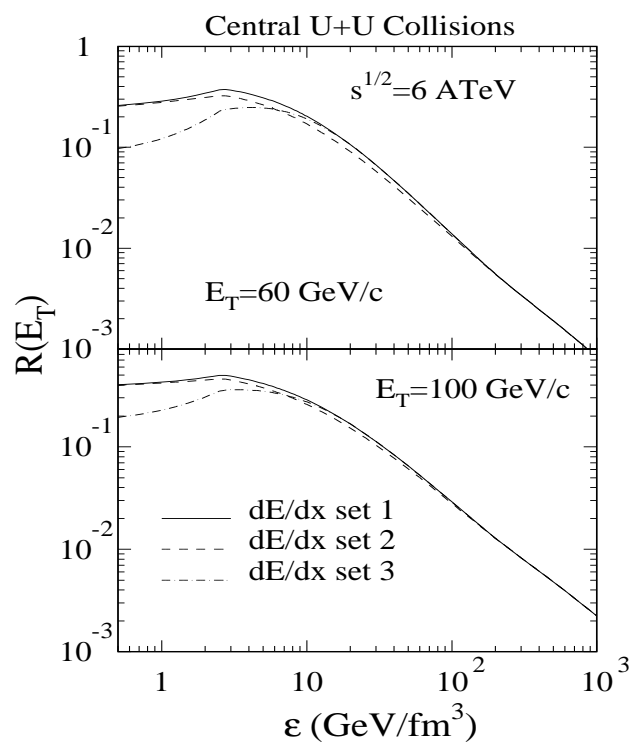

Figure 40: The same as Fig. 39, except at $\sqrt{s}=6 \mathrm{TeV}$ 
interaction with the dense medium,

$$
R_{A A}\left(E_{\mathrm{tot}}\right)=\frac{\left.\int d p_{T 1} \int d p_{T 2} \delta\left(E_{\mathrm{tot}}-p_{T 1}-p_{T 2}\right) \frac{d N_{\text {dijet }}}{d p_{T 1} d p_{T 2} d y_{1} d y_{2}}\right|_{y_{1}=y_{2}=0}}{\left.\int d p_{T}^{2} \delta\left(E_{\mathrm{tot}}-2 p_{T}\right) \frac{d N_{\mathrm{dijet}}^{(0)}}{d p_{T}^{2} d y_{1} d y_{2}}\right|_{y_{1}=y_{2}=0}}
$$

for dijets at $y_{1}=y_{2}=0$ in the CM frame,

Figs. 39 and 40 show the dependence of the quenching rate on the initial energy density $\varepsilon_{0}$ for central $U+U$ collisions at $\sqrt{s}=200 \mathrm{AGeV}$ and $\sqrt{s}=6 \mathrm{ATeV}$, respectively, for given total dijet energies. The results were obtained by evaluating Eqs. (174) and (175) for the Duke-Owens structure functions set 1 [55] and the EOS discussed above. The initial proper time was taken to be $\tau_{0}=1 / T_{0}$. The nontrivial dependence of $R_{A A}\left(E_{\text {tot }}\right)$ on $\varepsilon_{0}$ reflects the temperature dependence of $d E / d z$ as shown in Fig. 38. For small initial energy densities, the high energy partons of the dijet suffer a temperature-independent energy loss per unit distance, $d E / d z=1$ $\mathrm{GeV} / \mathrm{fm}$, in the hadronic phase of the dense matter at temperatures well below $T_{c}$. As $\varepsilon_{0}$ increases the partons will spend more and more time in the mixed phase. For all scenarios,$d E / d z$ takes its minimum value in the mixed phase and consequently, the quenching rate increases with increasing initial energy density, i.e., one observes less quenching. On the other hand, in the absence of such a local minimum, no increase of the dijet rate with $\varepsilon_{0}$ occurs. As $\varepsilon_{0}$ further increases, the leading partons of the jet probe the QGP phase at higher temperatures. Since $d E / d z$ grows with $T$ for $T>T_{c}, R_{A A}\left(E_{\text {tot }}\right)$ decreases with $\varepsilon_{0}$ in this region. All three curves come together asymptotically as they correspond to the same $d E / d z$ in the QGP phase. Thus a maximum of $R_{A A}\left(E_{\text {tot }}\right)$ at intermediate $\varepsilon_{0}$ reflects a minimum of $d E / d z$ at the critical temperature of the phase transition.

\subsubsection{Monojet production}

Depending on the position of the initial hard parton scattering and the direction of their momenta after the interaction, it can happen that one of the high energy partons has to traverse a large distance in the dense matter while the other one travels only a short distance in the medium. If the first parton loses such a large amount of energy that the corresponding jet can no longer be distinguished from the soft and semi-hard

background, one observes a mono-jet. To be specific, let us define a mono-jet as a dijet where one of the jets has a transverse momentum below a given value $p_{T \text { cut }}$. The number of mono-jets of transverse momentum $p_{T}$ and rapidity $y$ per central $A A$ 


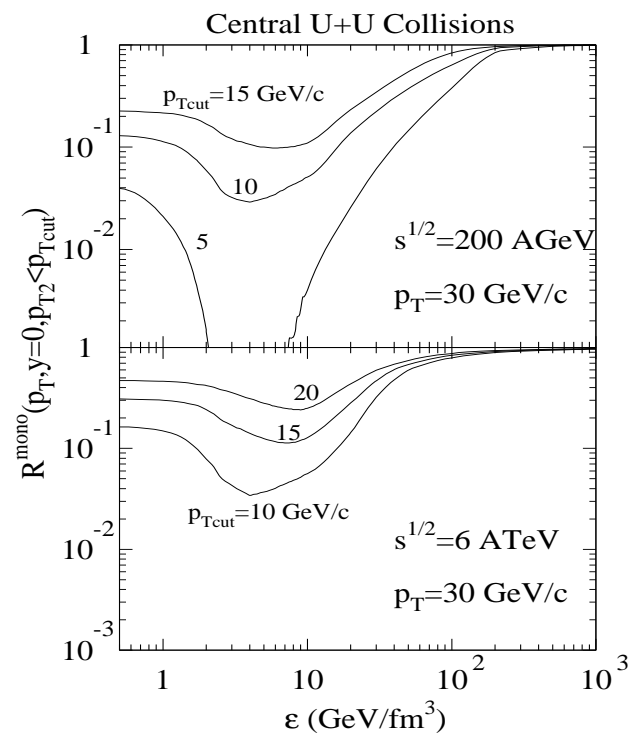

Figure 41: Monojet fraction as a function of the initial energy density $\varepsilon_{0}$ with a fixed $p_{T}=30 \mathrm{GeV}$ and different values of $p_{T \text { cut }}$ for central $U+U$ collisions at $\sqrt{s}=200$ $\mathrm{GeV}$, and $6 \mathrm{TeV}$.

collision at $y=0$ is given by [cf. Eq. (175)]

$$
\left.\frac{d N_{\text {mono }}\left(p_{T 2}<p_{T \text { cut }}\right)}{d p_{T} d y}\right|_{y=0}=\left.\int d y_{2} \int d p_{T 2} \Theta\left(p_{T \text { cut }}-p_{T 2}\right) \frac{d N_{\text {dijet }}}{d p_{T} d p_{T 2} d y d y_{2}}\right|_{y=0}
$$

where one has to integrate over the (experimentally unknown) rapidity and transverse momentum of the second parton.

For large $\varepsilon_{0}$, the number of mono-jets must decrease because of the increase of $d E / d z$ with temperature in the QGP phase which leads to the suppression of all jets. In this context it is useful to define the mono-jet fraction, i.e., the mono-jet rate divided by the total jet rate, at a given transverse momentum $p_{T}$ and rapidity $y=0$,

$$
R_{\mathrm{mono}}\left(p_{T}, p_{T 2}<p_{T \mathrm{cut}}, y=0\right)=\frac{\left.\int d y_{2} \int d p_{T 2} \Theta\left(p_{T \mathrm{cut}}-p_{T 2}\right) \frac{d N_{\text {dijet }}}{d p_{T} d p_{T 2} d y d y_{2}}\right|_{y=0}}{\left.\int d y_{2} \int d p_{T 2} \frac{d N_{\text {dijet }}}{d p_{T} d p_{T 2} d y d y_{2}}\right|_{y=0}}
$$

In Fig. 41, $R_{\text {mono }}\left(p_{T}=30 \mathrm{GeV} / \mathrm{c}, p_{T 2}<p_{T \mathrm{cut}}, y=0\right)$ is plotted as a function of $\varepsilon_{0}$ for scenario 1. Clearly, the unquenching of jets associated with the minimum of $d E / d z$ at the critical temperature leads to a dip in the mono-jet rate which corresponds to 
the peak in the di-jet quenching rate (cf. Figs. 39 and 40). In the limit of large initial energy densities $R_{\text {mono }} \rightarrow 1$, i.e., all jets are mono-jets at large $\varepsilon_{0}$. The reason is that eventually $d E / d z$ becomes so large that, except for negligible fraction of parton scatterings whose production points are close to the nuclear surface in the transverse plane, only one of the two jets can survive.

Since the jet production rates depend on energy, $A$ which determine the transverse size of the dense matter, $R_{A}$, and the initial energy density $\varepsilon_{0}$, one would like to fixed the first two variables and isolate only the dependence on $\varepsilon_{0}$. In experiments, there is no practical method to vary the initial energy density $\varepsilon_{0}$ for both fixed $\sqrt{s}$ and $A$. In order to best study jet quenching and monojet production as a probe of the phase transition, one can vary the initial energy density by either changing $\sqrt{s}$ for fixed beams or changing $A$ number for fixed energy [110].

\section{Summary and Discussion}

In this report, I presented a pQCD-based picture of ultrarelativistic heavy-ion collisions. In this framework, a nucleus in the infinite momentum frame consists of many partons (quarks and gluons). The interactions among these partons can be divided into perturbative, which can be described by pQCD calculation, and nonperturbative, which can only be modeled phenomenologically. I have demonstrated that pQCD processes dominate the underlying dynamics of heavy-ion collisions at extremely high energies. I argued that the soft component of a Pomeron exchange would be suppressed in the presence of a dense and hot partonic plasma, though it is still important in the initial parton scatterings in the early stage of the heavy ion collisions and is responsible for the initial soft parton production. It is then reasonable to assume that the evolution of the initially produced partons can be described by pQCD processes. Using the initial conditions estimated by the HIJING Monte Carlo model, the following picture emerges:

(1) During the early stage in ultrarelativistic heavy-ion collisions, hard or semihard parton scatterings, which happen in a time scale of about $0.2 \mathrm{fm} / c$, produce a hot and undersaturated parton gas. This parton gas is dominated by gluons and is far from chemical equilibrium. Multiple hard scatterings suffered by a single parton during this short period of time when the beam partons pass through each other are suppressed due to the interference embedded in the Glauber formula for multiple scatterings. This leads to the predicted disapparence of the Cronin effect. Interference and parton fusion also lead to the depletion of small $x$ partons in the effective parton distributions inside a nucleus. This nuclear shadowing of parton distributions reduces the initial parton production. 
(2) After the two beams of partons pass through each other, the produced parton gas in the central rapidity region starts its evolution toward (kinetic) thermalization and (chemical) equilibration through elastic scatterings and induced radiations. The kinematic separation of partons in the central slab of about $1 \mathrm{fm}$ through free-streaming gives an estimate of the time scale $\tau_{\text {iso }} \sim 0.5-0.7 \mathrm{fm} / c$, when local isotropy in momentum distributions is reached. Further evolution of the parton gas toward a fully equilibrated parton plasma is dictated by the parton proliferation through induced radiation and gluon fusion. Though the gluon equilibration rate is reduced by the inclusion of the Landau-Pomeranchuk-Migdal effect, gluon fugacity still increases rapidly toward its equilibrium value. Due to the consumption of energy by the additional parton production, the effective temperature of the parton plasma cools down considerably faster than the ideal Bjorken's scaling solution. Therefore, the life time of the plasma is reduced to $4-6 \mathrm{fm} / c$ before the temperature drops below the QCD phase transition temperature.

(3) The evolution of the quark distribution always lags behind that of gluons due to a smaller equilibration rate and the initial density. For heavy quarks, the equilibration rate is even smaller. Take charm quarks for example. The thermal production during the equilibration period is much smaller than the initial direct production, due to the small initial gluon fugacity and the short life time during which the temperature remains high enough to produce charm quarks. For the same reason, dilepton and photon production during the evolution of the parton plasma is also small because of small quark number density. Therefore, observation of large charm and dilepton enhancement would imply high initial gluon and quark density and thus a longer life time of the parton plasma [143, 144, 145].

(4) Even though the initial parton system is not in a full equilibrium, a study of color screening [135] shows that the system is already in a deconfined state with large average momentum (or effective temperature). Such a deconfined parton system, though not in equilibrium, will dissociate hadronic states like $J / \psi$. It was shown that $J / \psi$ can be substantially suppressed during the evolution of the parton plasma toward equilibrium. The measurements of this suppression can reveal the initial conditions and the evolution history of the parton plasma in high-energy heavy-ion collisions.

Readers should be reminded that the above picture is the result of only a qualitative estimate based on a pQCD-based model. Many uncertainties prevent us from making a more quantitative calculation. All in all, these uncertainties arise from our ignorance of the nonperturbative physics and our inability to calculate the soft processes in the framework of QCD. In the pQCD-based model reviewed here, the uncertainties really lie in the cut-off, $p_{0}$, which is supposed to separate nonperturbative soft interactions from perturbative hard processes. Since soft and hard physics do 
not have a definite boundary, the resultant parton production from hard or semihard processes is very sensitive to the cut-off. The accompanying soft parton production is not known in this model and may only be estimated by effective models like the color flux-tube model [61. Recently, it has been argued that the presence of larger $p_{T}$ partons can provide screening to the parton production with smaller $p_{T}$ [180. The screening mass can then replace the ad hoc cut-off and regulates the parton production cross section self-consistently. There have been recent developments in the field theory of particle production from mean fields [24]. Such a field theoretical approach to particle production could be the ultimate and consistent way to address the production and formation of a parton plasma in heavy-ion collisions, once the collision terms are included and the theory is generalized to the QCD case with rather random color fields.

The other nonperturbative uncertainty comes from the parton distribution functions inside a nucleus. Though quark distributions have been measured in deeply inelastic lepton-nucleon (nucleus) collisions, the gluon distribution is not known very well. Furthermore, nuclear modification of the gluon distribution has not at all been measured experimentally. Since gluon scatterings are the dominant QCD processes in parton production, our results are very sensitive to the gluon distribution. On top of that, nuclear shadowing will cause additional $20-50 \%$ uncertainty in the initial parton density. One way to reduce this uncertainty is to measure $p p$ and $p A$ collisions systematically as proposed in Ref. 88, 159.

For a thermalized parton plasma, the uncertainties related to nonperturbative physics will be reduced, since the soft processes (long distance interactions) will be screened by the interactions with the hot medium. For example, the original divergent cross sections are regularized by the color screening mass $\mu_{D}$ and the infrared and collinear singularities associated with gluon radiations can be regularized by the LPM suppression. Recent developments in QCD transport theory [25] have systematically studied these problems and formally included the resummation of hard thermal loops, first proposed by Braaten and Pisarski [133] in finite temperature field theory. However, one finds that, even though the electric sector of the strong interaction is regularized by an effective Debye screening mass, the magnetic interaction is still not regularized. For most of the transport coefficients, this is not a problem since the cancellation between in and out-state scatterings makes the effective cross section less divergent and the imaginary part of the gluon self-energy is enough to regulate the infrared divergency [132, 130, 122]. This is so-called dynamic screening by Landau damping. For some other quantities, like gluon damping rate 181 and color diffusion rate [131, 132], one still has to invoke a magnetic screening mass which will always be a nonperturbative quantity. Higher order corrections to the Debye screening mass 
also depend on the non-perturbative magnetic screening mass [182]. Therefore, some aspect of the parton thermalization and equilibration are still influenced by nonperturbative physics.

Throughout the production and evolution of the partonic system, interference effects play an important role in multiple collision processes, the correct implementation of which will be a great challenge to any transport model based on classical parton cascades. First, the interference between different amplitudes in multiple parton scatterings leads to the Glauber formula which suppresses multiple hard scatterings of beam partons. Second, the LPM effect, i.e., the destructive interference among different amplitudes of gluon radiation induced by multiple scatterings, suppresses soft gluon radiation whose effective formation time, $\tau_{\mathrm{QCD}}$, is larger than the mean free path, $\lambda_{f}$, of the parton scatterings. This leads both to reduced gluon equilibration rates and reduced radiative energy loss. A detailed analysis of the radiation amplitude has revealed the underlying physics which contradicts the intuitive picture of the classical parton cascade model. A propagating parton can no longer be considered as time-like with a degrading virtuality as it radiates between multiple scatterings, since the parton can be space-like $\left(q^{2}<0\right.$ for initial state radiation) in one amplitude and time-like $\left(q^{2}>0\right.$ for final state radiation) in another. It is the cancellation between these amplitudes that suppresses the soft radiation with long formation time. One way to incorporate the LPM effect in the parton interaction simulation, as I have illustrated, is to consider both the initial and final state radiation together for each scattering and impose the formation time requirement, $\tau_{\mathrm{QCD}}<\lambda_{f}$, for the integration over the phase-space of the radiated gluons. Shuryak and Xiong [141] have used multiple gluon production amplitudes given by Parke and Taylor [138. This approach has improved the gluon radiation calculation beyond the leading logarithm approximation. However, the space-time structure of the multiple gluon amplitudes is not clear and a similar LPM analysis cannot be performed. In addition, virtual corrections have to be included to satisfy the unitarity requirement.

In conclusion, pQCD-based models predict the formation of a hot and dense parton plasma in ultrarelativistic heavy-ion collisions, in which color deconfinement can be achieved and gluons dominate the number of degrees of freedom. The answer to the question whether such a system can finally evolve into a fully thermalized and equilibrated parton plasma still depends crucially on the reduction of the uncertainties involved and the understanding of the underlying nonperturbative physics. Although theoretical progress can and must be made in these directions, the ultimate quantitative solution still awaits the experimental measurements at RHIC and LHC. 


\section{Acknowledgements}

I am deeply indebted to M. Gyulassy for his encouragement and constant flow of ideas which have inspired most of the work I discussed here, and his enthusiastic collaboration in the development of the HIJING model. I thank K. J. Eskola for his collaboration which I have enjoyed very much. I am grateful to B. Müller for his inspiring collaboration through the last few years. I would also like to thank some of my collaborators, T. S. Biró, D. Kharzeev, P. Lévai, J. Qiu, H. Satz, M. H. Thoma and X.-M. Xu who made significant contributions to the work I reviewed in this paper. Stimulating discussions with K. Geiger, H. Heiselberg, J. I. Kapusta, L. McLerran, E. Shuryak, and L. Xiong are also gratefully acknowledged. Finally, I would like to thank Ulrich Heinz for his comments and critical reading of this review.

\section{References}

[1] J. C. Collins and M. Perry, Phys. Lett. 34, 1353 (1975); G. Baym and S. A. Chin, Phys. Lett. 62 B, 241 (1986); B. Friedman and L. McLerran, Phys. Rev. D 17, 1109 (1978).

[2] S. Shuryak, Phys. Rep. 61, 71 (1980); D. Gross, R. Pisarski and L. Yaffe, Rev. Mod. Phys. 53, 43 (1981); H. Satz, Ann. Rev. Nucl. Part. Sci. 35, 245 (1985); L. McLerran, Rev. Mod. Phys. 58, 1021 (1986).

[3] F. Karsch, E. Laermann, Rept. Prog. Phys. 56, 1347 (1993); N. H. Christ, Nucl. Phys. A544, 81c (1992); Y. Iwasaki, et al., Phys. Rev. Lett. 67, 3343 (1991); F. R. Brown, et al., Phys. Rev. Lett. 65, 2491 (1990).

[4] See, e.g., review and summary talks by B. Müller and M. Gyulassy in Quark Matter'95, proceedings of the 11th International Conference on UltraRelativistic Nucleus-Nucleus Collisions, Monterey, California, USA, 1995, eds. A. M. Poskanzer, J. W. Harris and L. S. Schroeder. [Nucl. Phys. bf A590, 1c (1995)].

[5] See, e.g., J. W. Harris; J. H. Thomas; H. H. Gutbrod, in the proceedings of the workshop on Pre-Equilibrium Parton Dynamics, Berkeley, 1993, edited by X.-N. Wang, Report LBL-34831.

[6] H. H Gutbrod, A. M. Poskanzer, H. G. Ritter, Rep. Prog. Phys. 52, 267 (1989).

[7] J. Barrette et al., Phys. Rev. Lett. 73, 2532 (1994).

[8] X.-N. Wang and M. Gyulassy, Phys. Rev. D 45, 844 (1992).

[9] J.P. Blaizot, A.H. Mueller, Nucl. Phys. B289, 847 (1987).

[10] K. Kajantie, P. V. Landshoff and J. Lindfors, Phys. Rev. Lett. 59, 2517 (1987); K. J. Eskola, K. Kajantie and J. Lindfors, Nucl. Phys. B323, 37 (1989); K. J. Eskola, K. Kajantie and J. Lindfors, Phys. Lett. B 214, 613 (1989). 
[11] A. Jahns, C. Spieles, H. Sorge, H. Stöcker and W. Greiner, Phys. Rev. Lett. 72, 3464 (1994); H. Sorge, R. Mattiello, H. Stöcker and W. Greiner, ibid. 68, 286 (1992).

[12] Y. Pang, T. J. Schlagel, S. H. Kahana, Phys. Rev. Lett. 68, 2743 (1992); 69, 3290 (1992).

[13] B. Andersson, G. Gustafson and B. Nilsson-Almqvist, Nucl. Phys. B281, 289 (1987); B. Nilsson-Almqvist and E. Stenlund, Comp. Phys. Comm. 43, 387 (1987).

[14] A. Capella, U. Sukhatme and J. Tran Thanh Van, Z. Phys. C3, 329 (1980); J. Ranft, Phys. Rev. D 37, 1842 (1988); Phys. Lett. 188B, 379 (1987).

[15] M. Gyulassy, Proceedings of Eighth Balaton Conference on Nuclear Physics, edited by Z. Fodor (KFKI, Budapest, 1987); CERN preprint CERN-TH4794/87(1987).

[16] K. Werner, Z. Phys. C42, 85 (1989); Phys. Rep. 232, 87 (1993).

[17] T. Sjöstrand and M. van Zijl, Phys. Rev. D 36, 2019 (1987); T. Sjöstrand, Comp. Phys. Commun. 39, 347 (1986); T. Sjöstrand and M. Bengtsson, ibid. 43, 367 (1987).

[18] F. E. Paige, S. D. Protopopescu, in the proceedings of the Summer Study of the Physics of the SSC, Snowmass, 1986, edited by R. Donaldson and J. Marx.

[19] X.-N. Wang and M. Gyulassy, Phys. Rev. D 44, 3501 (1991); Comp. Phys. Commun. 83, 304 (1994).

[20] K. Geiger and B. Müller, Nucl. Phys. B369, 600 (1992); K. Geiger, Phys. Rev. D 47, 133 (1993); K. Geiger, Phys. Rep. 258, 237 (1995).

[21] H. J. Moehring and J. Ranft, Z. Phys. C 52, 643 (1991); P. Aurenche, et al., Phys. Rev. D45, 92 (1992). P. Aurenche, et al., Comp. Phys. Commun. 83, 107 (1994).

[22] D. Boal, Phys. Rev. C 33, 2206 (1986).

[23] H. T. Elze and U. Heinz, Phys. Rep. 183, 81 (1989); S. Mrówczyǹski and U. Heinz, Ann. Phys. 229, 1 (1994).

[24] Y. Kluger, J. M. Eisenberg, B. Svetitsky, F. Cooper and E. Mottola, Phys. Rev. Lett. 67, 2427 (1991); Phys. Rev. D 45, 4659 (1992); ibid. D 48, 190 (1993).

[25] J.-P. Blaizot and E. Iancu, Nucl. Phys. B390, 589 (1993); Phys. Rev. Lett. 70, 3376 (1993).

[26] R. J. Glauber, in Lectures in Theoretical Physics, edited by E. Brittin et al. (Interscience, New York, 1959), Vol. I.

[27] M. Gyulassy and X.-N. Wang, Nucl. Phys. B 420, 583 (1994).

[28] S. J. Brodsky and H. J. Lu, Phys. Rev. Lett. 64, 1342 (1990). 
[29] L. D. Landau and I. J. Pomeranchuk, Dolk. Akad. Nauk. SSSR 92, 92(1953); A. B. Migdal, Phys. Rev. 103, 1811 (1956); E. L. Feinberg and I. J. Pomeranchuk, Suppl. Nuovo. Cimento 3, 652 (1956); Phys. JETP 23, 132 (1966).

[30] A. I. Akhiezer and N. F. Shulga, Sov. Phys. Usp. 30, 197 (1987).

[31] X.-N. Wang, M. Gyulassy and M. Plümer, Phys. Rev. D 51, 3436 (1995).

[32] Yu. L. Dokshitzer, D. Schiff, S.Peigné and R. Baier, Phys. Lett. B356, 349 (1995).

[33] A. Capella and J. Tran Thanh Van, Z. Phys. C23, 165 (1984); T. K. Gaisser and F. Halzen, Phys. Rev. Lett. 54, 1754 (1985); P. l'Heureux, et al., Phys. Rev. D 32, 1681 (1985); G. Pancheri and Y. N. Srivastava, Phys. Lett. 182B, 199 (1986); L. Durand and H. Pi, Phys. Rev. Lett. 58, 303 (1987); J. Dias de Deus and J. Kwiecinski, Phys. Lett. 196B, 537 (1987); R. C. Hwa, Phys. Rev. D 37, 1830 (1988).

[34] X. N. Wang and R. C. Hwa, Phys. Rev. D 39, 187 (1989).

[35] X. N. Wang, Phys. Rev. D 43, 104 (1991).

[36] The Pomeron, proceedings of VII Rencontre de Moriond, Meribel-Les-Allues, March 4-16, 1973, Vol. II, edited by J. Tran Thanh Van, Orsay 1975.

[37] L. V. Gribov, E. M. Levin and M. G. Ryskin, Phys. Reports 100, 1 (1983); Nucl. Phys. B188, 555 (1981); Zh. Eksp. Teor. Fiz. 80, 2132 (1981).

[38] A. Donnachie and P. V. Landshoff, Nucl. Phys. B267, 690 (1986); ibid. B244, 322 (1984); Phys. Lett. 296B, 227 (1992).

[39] See, e.g., L. I. Schiff, Quantum Mechanics (McGraw-Hill, New York, 1964).

[40] N. Amos et al., Nucl. Phys. B 262, 689 (1985); L. A. Fajardo et al., Phys. Rev. D 24, 461 (1987).

[41] X.-N. Wang, Ph. D. Thesis, University of Oregon, 1989.

[42] V. N. Gribov and L. N. Lipatov, Sov. J. Nucl. Phys. 15, 78 (1971); G. Altarelli and G. Parisi, Nucl. Phys. B 126, 289 (1977).

[43] E. Eichten, I. Hinchliffe, K. Lane and C. Quigg, Rev. Mod. Phys. 56, 579 (1984).

[44] S. D. Ellis, Z. Kunszt and D. E. Soper, Phys. Rev. Lett. 62, 726 (1989); Phys. Rev. D 40, 2188 (1989).

[45] K. J. Eskola and X.-N. Wang, in Hard Processes in Hadronic Interactions, eds. H. Satz and X.-N. Wang, Int. J. Mod. Phys. A 10, 3071 (1995).

[46] A. Donnachie and P. V. Landshoff, preprint DAMTP-94-28.

[47] L. Durand and H. Pi, Phys. Rev. D 38, 78 (1988); T. K. Gaisser and T. Stanev, Phys. Lett. 219B, 375 (1989).

[48] Z. Koba, H. N. Nielsen and P. Olesen, Nucl. Phys. B40, 317 (1972); U. Amaldi and K. R. Schubert, Nucl. Phys. B166, 301 (1980).

[49] J. Dias de Deus, Nucl. Phys. B59, 231 (1973); B252, 369 (1985). 
[50] UA4 Collab., M. Bozzo et al., Phys. Lett. 147B, 392 (1984).

[51] UA5 Collab., G. J. Alner, et al., Z. Phys. C32, 153 (1986).

[52] Fermilab E710 Collab., N. Amos et al., Phys. Rev. Lett. 63, 2784 (1989).

[53] R. M. Baltrusaitis et al., Phys. Rev. Lett. 52, 1380 (1984).

[54] T. Hara et al., Phys. Rev. Lett. 50, 2058 (1983). The $p p$ cross sections converted from $p$-air data are taken from L. Durand and H. Pi, Phys. Rev. Lett. 58, 303 (1987).

[55] D. W. Duke and J. F. Owens, Phys. Rev. D 30, 50 (1984).

[56] M. Geück, E. Reya and A. Vogt, Z. Phys. C 53, 127 (1993).

[57] A. D. Martin, W. J. Stirling and R. G. Roberts, Phys. Lett. 306B, 145 (1993).

[58] ZEUS Coll., M. Derrick et al., Phys. Lett. 316B, 412 (1993).

[59] K. J. Eskola, K. Kajantie and P. V. Ruuskanen, Phys. Lett. B332, 191 (1994).

[60] K. J. Eskola and X.-N. Wang, work in progress.

[61] K. J. Eskola and M. Gyulassy, Phys. C 47, 2329 (1993).

[62] B. Andersson, G. Gustafson, G. Ingelman and T. Sjöstrand, Phys. Rep. 97,31 (1983).

[63] T. Sjöstrand, Comput. Phys. Commun. 27, 243 (1982).

[64] British-Scandinavian Collab., B. Alper, et al., Nucl. Phys. B87, 19 (1975).

[65] UA1 Collab., C. Albajar, et al., Nucl. Phys. B335, 261 (1990).

[66] F. Abe, et al., Phys. Rev. Lett. 61, 1819 (1988).

[67] UA5 Collab., G. J. Alner, et al., Z. Phys. C 33, 1 (1986).

[68] F. Abe, et al., Phys. Rev. D 41, 2330 (1990).

[69] ABCDHW Collab., A. Breakstone, et al., Phys. Rev. D 30, 528 (1984).

[70] UA5 Collab., R. E. Ansorge, et al., Z. Phys. C 43, 357 (1989).

[71] UA5 Collab., G. J. Alner, et al., Phys. Rep. 154, 247 (1987).

[72] W. Thomé, et al., Nucl. Phys. B129, 365 (1977).

[73] J. Whitemore, et al., Phys. Rep. 10C, 273 (1974); W. M. Morse, et al., Phys. Rev. D 15, 66 (1977); C. P. Ward, et al., Nucl. Phys. B153, 299 (1979).

[74] X.-N. Wang and M. Gyulassy, Phys. Rev. D 45, 844 (1992).

[75] T. Alexopoulos, et al., Phys. Rev. Lett. 64, 991 (1990).

[76] G. Baym, B. L. Friman, J. P. Blaizot, M. Soyeur, and W. Czyz, Nucl. Phys. A407, 541 (1983); M. Kataja, P. V. Ruuskanen, L. D. McLerran, and H. von Gersdorff, Phys. Rev. D 34, 2755 (1986); X.-N. Wang and R. C. Hwa, Phys. Rev. D 35, 3409 (1987).

[77] P. Lévai and B. Müller, Phys. Rev. Lett. 67, 1519 (1991).

[78] L. van Hove, Z. Phys. C 21, 93 (1983).

[79] X.-N. Wang and M. Gyulassy, Phys. Lett. B282, 466 (1992).

[80] S. L. Wu, Phys. Rep. 107, 59(1984). 
[81] C. Merino, C. Pajares, and J. Ranft, Phys. Lett. B 276, 168 (1992).

[82] X.-N. Wang, Phys. Rev. D 46, R1900 (1992); D 47, 2754 (1993).

[83] UA1 Collaboration, G. Arnison et al., Phys. Lett. B172, 461 (1986); C. Albajar et al., Nucl. Phys. B309, 405 (1988).

[84] A. Byon, CDF Ph.D. thesis, Purdue University.

[85] AFS Collaboration, T. Akesson et al., Z. Phys. C 30, 27 (1986); and the reference therein.

[86] P. Bordalo et al., Phys. Lett. B 193, 373 (1987); D. M. Alde et al., Phys. Rev. Lett. 64, 2479 (1990).

[87] K. J. Eskola, Z. Phys. C 51, 633 (1991).

[88] X.-N. Wang and M. Gyulassy, Phys. Rev. Lett. 68, 1480 (1992).

[89] EM Collaboration, M. Arneodo et al., Nucl. Phys. B333 1 (1990); NM Collaboration, P. Amaudruz et al, Z. Phys. C 51387 (1991); E665 Collaboration, M. R. Adams et al., Phys. Rev. Lett. 68, 3266 (1992); Phys. Lett. B287, 375 (1992).

[90] N.N. Nikolaev and V.I. Zakharov, Phys. Lett. B55, 297 (1975); Z. Phys. C 49, 607 (1991); Phys. Lett. B260, 414(1991); Z. Phys. C 53, 331 (1992).

[91] L. L. Frankfurt and M.I. Strikman, Phys. Rep. 160, 237 (1988); Nucl. Phys. B316, 340 (1989); L. L. Frankfurt, M. I. Strikman and S. Liuti, Phys. Rev. Lett. 65, 1725 (1990).

[92] F. E. Close, J. Qiu and R.G. Roberts, Phys. Rev. D40, 2820 (1989).

[93] P. Castorina and A. Donnachie, Z. Phys. C 45, 141 (1989); J. Kwieciński, Z. Phys. C 45, 45 (1990).

[94] L. McLerran and R. Venugopalan, Phys. Rev. D 49, 2233 (1994); 3352 (1994); D 50, 2225 (1994).

[95] A. H. Mueller and J. Qiu, Nucl. Phys. B268, 427 (1986).

[96] J. Collins and J. Kwieciński, Nucl. Phys. B335, 89 (1990).

[97] See, e.g., R. D. Field, Applications of Perturbative QCD, Frontiers in Physics, Vol. 77 (Addison-Wesley, 1989).

[98] J. C. Collins and J. Qiu, Phys. Rev. D 39, 1398 (1989).

[99] J. Kwieciński, A. D. Martin, W. J. Stirling and R. G. Roberts, Phys. Rev. D 42, 3645 (1990).

[100] K. J. Eskola, J. Qiu and X.-N. Wang, Phys. Rev. Lett. 72, 36 (1994).

[101] J. Botts, et. al., Phys. Lett. B304, 159 (1993).

[102] K. J. Eskola, Nucl. Phys. B400, (1993) 240.

[103] P. B. Straub et al., Phys. Rev. Lett. 68, 452 (1992).

[104] M. Lev and B. Petersson, Z. Phys. C 21, 155 (1983).

[105] C. W. Dejager, H. DeVries and C. DeVries, Atomic Data and Nuclear Data Tables, 14, 479 (1974). 
[106] R. Odorico, Nucl. Phys. B172, 157 (1980).

[107] G. Marchesini and B. R. Webber, Nucl. Phys. B238, 1 (1984).

[108] M. Gyulassy and M. Plümer, Phys. Lett. 243B, 432 (1990).

[109] M. Gyulassy, M. Plümer, M. H. Thoma and X.-N. Wang, Nucl. Phys. A 538, 37c (1992).

[110] M. Plümer, M. Gyulassy, X.-N. Wang, Proceedings of Quark Matter'95, Monterey, Jan. 9, 1995, edited by A. M. Poskanzer, J. W. Harris and L. S. Schroeder.

[111] C. De Marzo, et al., Phys. Rev. D 26, 1019 (1982).

[112] NA35 Collab., H. Ströbele, et al., Z. Phys. C 38, 89 (1988)

[113] S. Gavin and P. V. Ruuskanen, Phys. Lett. B262, 326 (1991).

[114] G. M. Welke and G. F. Bertsch, Phys. Rev. C 451403 (1992).

[115] J. Collins, D. E. Soper, and G. Sterman, Nucl. Phys. B261, 104 (1985).

[116] K. J. Eskola and X.-N. Wang, Phys. Rev. D 49, 1284 (1994).

[117] A. Mueller, Nucl. Phys. A498, 41c (1988).

[118] J. D. Bjorken, Phys. Rev. D 27, 140 (1983).

[119] T. S. Biró, E. van Doorn, B. Müller, M. H. Thoma, and X.-N. Wang, Phys. Rev. C 48, 1275 (1993).

[120] E. Shuryak, Phys. Rev. Lett. 68, 3270 (1992).

[121] R. C. Hwa and K. Kajantie, Phys. Rev. Lett. 56, 696 (1986).

[122] H. Heiselberg and X.-N. Wang, NORDITA-95/26 N, hep-ph/9504244 submitted to Phys. Rev. C; H. Heiselberg and X.-N. Wang, LBL-37688, to appear in Nucl. Phys. B.

[123] S. M. H. Wong, LPTHE-Orsay 96/07.

[124] K. Geiger and J. I. Kapusta, Phys. Rev. D 47, 4905 (1993).

[125] J. C. Collins, D. E. Soper and G. Sterman, Nucl. Phys. B263, 37 (1986).

[126] K. Geiger, Phys. Rev. D 48, 4129 (1993).

[127] J. Qiu and G. Sterman, Nucl. Phys. B353, 105 (1991).

[128] G. T. Bodwin, S. J. Brodsky and G. P. Lepage, Phys. Rev. D 39, 3287 (1989).

[129] T. Matsui, B. Svetitsky, and L. McLerran, Phys. Rev. D 34, 783 (1986).

[130] P. Danielewicz and M. Gyulassy, Phys. Rev. D 31, 53 (1985); A. Hosoya and K. Kajantie, Nucl. Phys. B250, 666 (1985); S. Gavin, Nucl. Phys. A435, 826 (1985).

[131] A. Selikov and M. Gyulassy, Phys. Lett. B 316, 316 (1993); Phys. Rev. C 49, 1726 (1994).

[132] H. Heiselberg, Phys. Rev. Lett. 72, 3013 (1994).

[133] E. Braaten and R. D. Pisarski, Nucl. Phys. B337, 569 (1990).

[134] T. S. Biró and B. Müller, Nucl. Phys. A561, 477 (1993).

[135] T. S. Biró, B. Müller, and X.-N. Wang, Phys. Lett. B283, 171 (1992). 
[136] T. Altherr and D. Seibert, Phys. Lett. B313, 149 (1993); ERRATUM ibid. B316, 633 (1993).

[137] V. V Klimov, Sov. Phys. JETP 55, 199 (1982); H.A. Weldon, Phys. Rev. D 26, 2789 (1982).

[138] S. J. Parke and T. R. Taylor, Phys. Rev. Lett. 56, 2459 (1986).

[139] Z. Kunszt and W. J. Stirling, Phys. Rev. D 37, 2439 (1988).

[140] F. A. Berends, R. Kleiss, P. De Causmaecker, R. Gastmans, and T. T. Wu, Phys. Lett. B103, 124 (1981).

[141] L. Xiong and E. Shuryak, Phys. Rev. C 49, 2207 (1994).

[142] B. Müller, Rept. Prog. Phys. 58, 611 (1995).

[143] B. Müller and X. N. Wang, Phys. Rev. Lett. 68, 2437 (1992).

[144] P. Lévai, B. Müller and X.-N. Wang, Phys. Rev. C, 51, 3326 (1995).

[145] Z. Lin and M. Gyulassy, Phys. Rev. C 51, 2177 (1995).

[146] P. L. McGaughey, E. Quack, P. V. Ruuskanen, R. Vogt and X.-N. Wang, in Hard Processes in Hadronic Interactions, eds. H. Satz and X.-N. Wang, Int. J. Mod. Phys. A 10, 2999 (1995).

[147] R. Vogt, S. J. Brodsky abd P. Hoyer, Nucl. Phys. bf B383, 643 (1992); S. J. Brodsky, P. Hoyer, A. H. Mueller and W. K. Tang, ibid. B 369, 519 (1992).

[148] CERES Collaboration, G. Agakichiev et al., Phys. Rev. Lett. 75, 1272 (1995).

[149] G. Q. Li, C. M. Ko, G. E. Brown, Phys. Rev. Lett. 75, 4007 (1995).

[150] P. V. Ruuskanen, in Particle Production in Highly Excited Matter, eds. H. H. Gutbrod and J. Rafelski, p593, NATO ASI Series B Vol. 303 (Plenum Press, 1993, New York).

[151] M. Asakawa, C. M. Ko, P. Levai, Phys. Rev. Lett. 70, 398 (1993).

[152] S. Gavin, S. Gupta, R. Kauffman, P. V. Ruuskanen, D. K. Srivastava and R. L. Thews, in Hard Processes in Hadronic Interactions, eds. H. Satz and X.-N. Wang, Int. J. Mod. Phys. A 10, 2961 (1995).

[153] K.J. Eskola and J. Lindfors, Z. Phys. C 46 C, 141 (1990).

[154] M. T. Strickland, Phys. Lett. B 331, 245 (1994).

[155] E. Shuryak and L. Xiong, Phys. Rev. Lett. 70, 2241 (1993).

[156] K. Geiger and J. I. Kapusta, Phys. Rev. Lett. 70, 1920 (1993).

[157] J. I Kapusta, P. Lichard and D. Seibert, Phys. Rev. D 44, 2774 (1991).

[158] R. Vogt, B. V. Jacak, P. L. McGaughey and P. V. Ruuskanen, Phys. Rev. D 49, 3345 (1994).

[159] Z. Lin and M. Gyulassy, Columbia preprint CU-TP-714.

[160] T. Matsui and H. Satz, Phys. Lett. B178, 416(1986).

[161] D. Kharzeev and H. Satz, Phys. Lett. B334, 155(1994).

[162] M. E. Peskin, Nucl. Phys. B156, 365(1979); G. Bhanot and M. E. Peskin, Nucl. Phys. B156, 391(1979). 
[163] A. Kaidalov, in QCD and High Energy Hadronic Interactions, J. Trân Thanh Vân (Ed.), Editions Frontieres, Gif-sur-Yvette, 1993.

[164] D. Kharzeev and H. Satz, in Quark-Gluon Plasma 2, R. C. Hwa (ed.) (World Scientific, Singapore), p.395.

[165] X.-M. Xu, D. Kharzeev, H. Satz and X.-N. Wang, preprint LBL-37985, CERNTH/95-304, to appear in Phys. Rev. C.

[166] F. Karsch and R. Petronzio, Phys. Lett. B193, 105(1987).

[167] S. Gavin and J. Milana, Phys. Rev. Lett. 68, 1834 (1992).

[168] D. Kharzeev and H. Satz, CERN-TH/95-214, August, 1995; Phys. Lett. B, to appear.

[169] R. Wittmann and U. Heinz, Z. Phys. C59, 77 (1993).

[170] S. Gavin, M. Gyulassy and A. Jackson, Phys. Lett. B208, 257 (1988); S. Gavin and M. Gyulassy, Phys. Lett. B214,241 (1988).

[171] D. A. Apple, Phys. Rev. D 33, 717 (1986); J. P. Blaizot and L. D. McLerran, Phys. Rev. D 34, 2739 (1986); M. Rammerstorfer and U. Heinz, Phys. Rev. D 41, 306 (1990).

[172] J. D. Bjorken, Fermilab-Pub-82/59-THY (1982) and erratum (unpublished).

[173] M. H. Thoma and M. Gyulassy, Nucl. Phys. B 351, 491 (1991).

[174] M. G. Ryskin, Sov. J. Nucl. Phys. 52, 139 (1990).

[175] A. H. Sorensen, Z. Phys. C 53, 595 (1992).

[176] S. J. Brodsky and P. Hoyer, Phys. Lett. B 298, 165 (1993).

[177] M. Gao, Phys. Rev. D 41, 626 (1990).

[178] F. Niedermayer, Phys. Rev. D 34, 3494 (1986).

[179] M. Gyulassy and M. Plümer, Nucl. Phys. B 346, 1 (1990); D.J. Dean et al., Phys. Rev. C 46, 2066 (1992).

[180] K. Eskola, B. Müller and X.-N. Wang, LBL-37642, hep-ph/9509285.

[181] E. Braaten and R. D. Pisarski, Phys. Rev. D 42, R2156 (1990); C. P. Burgess and A. L. Marini, Phys. Rev. D 45, R17 (1992); A. Rebhan, Phys. Rev. D 46, 482 (1992); H. Heiselberg and C. J. Pethick, Phys. Rev. D 48, R769 (1993).

[182] P. Arnold and L. G. Yaffe, preprint UW/PT-95-06, hep-ph/9508280. 